\title{
Chemical Manipulation of Honey Bee Behavior
}

\author{
Nicholas Ryan Larson
}

Dissertation submitted to the faculty of the Virginia Polytechnic Institute and State University in partial fulfillment of the requirements for the degree of

\author{
Doctor of Philosophy \\ In \\ Entomology
}

\author{
Troy D. Anderson \\ Carlyle C. Brewster \\ Ulrich R. Bernier \\ Jeffrey R. Bloomquist \\ Thomas P. Kuhar \\ Dorothea Tholl
}

May 3, 2017

Blacksburg, VA

Keywords: Honey bee, repellent, pollinator protection 


\title{
Chemical Manipulation of Honey Bee Behavior
}

\author{
Nicholas R. Larson
}

\begin{abstract}
The loss of managed honey bee colonies, resulting from their unintentional exposure to pesticides, is a topic of concern for the agricultural and apicultural industry. Current methods for reducing pesticide exposure to bees involve the application of pesticides before crop bloom or in the evening when foraging bees are less likely to be exposed to these applications. There is an urgent need for additional protection procedures to reduce the annual losses of managed bee colonies. Another method for protecting these pollinators is the use of chemical deterrents to reduce the interaction times of foraging bees with pesticide-treated crops. Historically, insect repellents (IRs) have been used to prevent the spread of deadly human diseases by arthropod vectors. However, it has been shown that bees can be repelled from pesticide-treated crops using DEET and bee pheromonal compounds. Here, I report the toxicological and deterrent effects of bee pheromonal compounds, as well as the deterrent effects of heterocyclic amines (HCAs) on bees. The results of this study indicate that the bee pheromonal compounds, at 8, 20,60 and 100\% concentrations, are toxic to bees and inhibit the feeding of bees within a confined space. Additionally, the pheromonal compounds and the HCAs are as efficacious as DEET in deterring bees from treated food sources. The HCA piperidine, was observed to effectively deter bee foragers from a sugar feeder in a hightunnel experiment as well as from melon flowers and knapweed in field experiments. Electroantennogram recordings were conducted to verify an olfactory response of the bees to the tested compounds. Pheromonal compounds were readily detected by bee antennae; whereas, the HCAs did not elicit significant responses in the bee antennae. These data suggest that bee
\end{abstract}


pheromonal compounds, as well as HCAs, may serve as candidates for the further investigation as repellents to protect bees from unintentional pesticide exposures. 


\section{Chemical Manipulation of Honey Bee Behavior}

Nicholas R. Larson

\section{GENERAL AUDIENCE ABSTRACT}

The loss of managed honey bee colonies, resulting from their unintentional exposure to pesticides, is a topic of concern for the agricultural and apicultural industry. Current methods for reducing pesticide exposures to bees involve the application of pesticides before crop bloom or in the evening when foraging bees are less likely to be exposed to these applications. There is an urgent need for additional protection procedures to reduce the annual losses of managed bee colonies. One method for protecting these pollinators is use of chemical deterrents to reduce the interaction times of foraging bees with pesticide-treated crops. Insect repellents (IRs) primarily have been used for biting and blood-feeding arthropod pests. However, it has been shown that bees can be repelled from pesticide-treated crops using DEET and bee pheromonal compounds. Here, I report the toxicological and deterrent effects of bee pheromonal compounds as well as the deterrent effects of heterocyclic amines (HCAs) on bees. The goals of this study were to: 1) examine the toxicological effects of the pheromonal compounds on bees, 2) develop a laboratory testing protocol for evaluating the deterrent effects of pheromonal bee compounds and heterocyclic amines (HCAs) to bees, 3) evaluate the deterrent effects of HCAs to bees using high-tunnel and semi-field experimentations, and 4) characterize the olfactory responses of bees to the above compounds. 


\section{TABLE OF CONTENTS}

INTRODUCTION

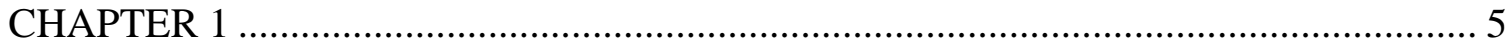

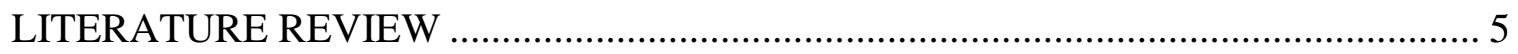

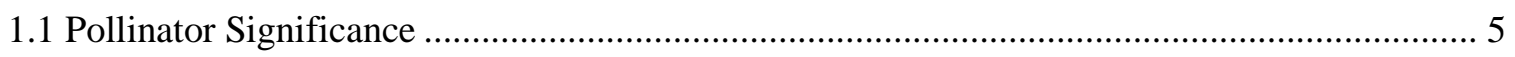

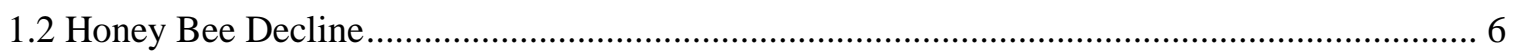

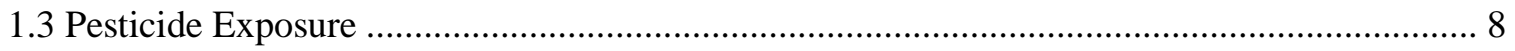

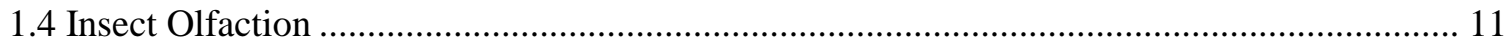

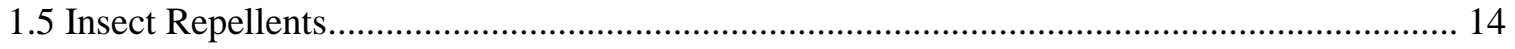

CHAPTER 2: EFFECTS OF HONEY BEE PHEROMONAL COMPOUNDS ON THE MORTALITY AND FEEDING BEHAVIOR OF HONEY BEES ................................. 21

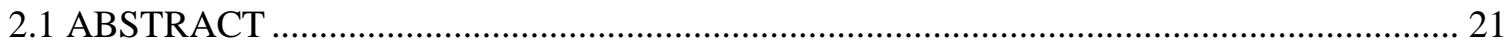

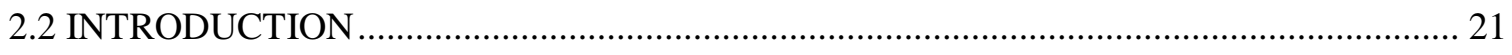

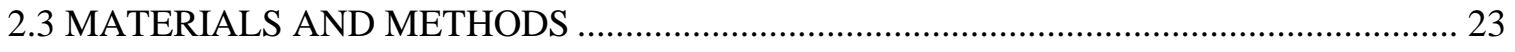

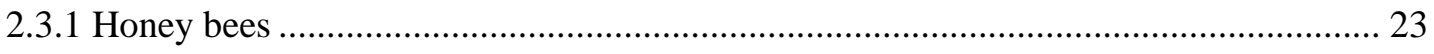

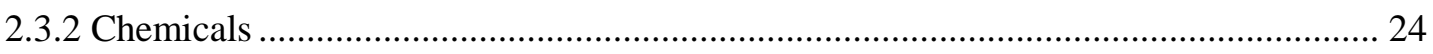

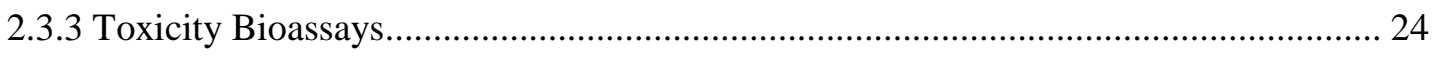

2.3.4 Feeding inhibition bioassays ......................................................................... 25

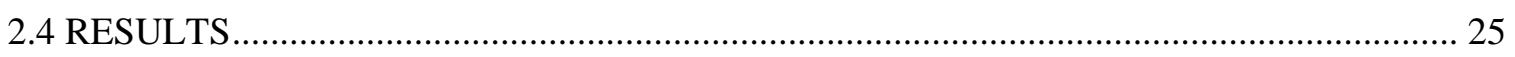

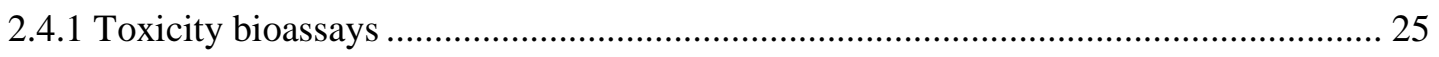

2.4.2 Feeding inhibition bioassays .......................................................................... 26

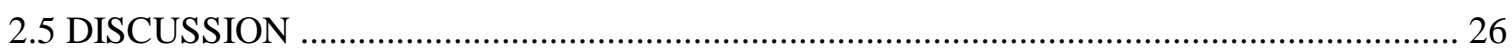

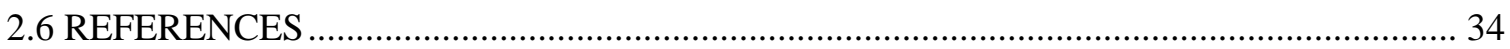

CHAPTER 3: VIDEO TRACKING PROTOCOL TO SCREEN DETERRENT

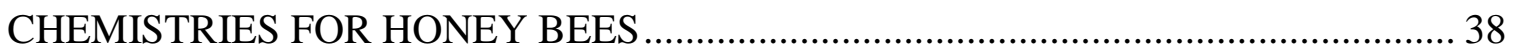

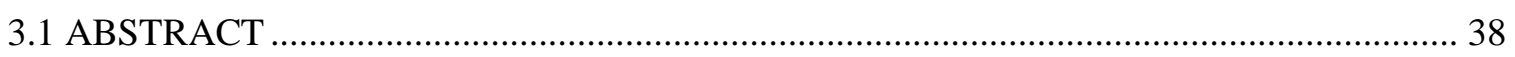

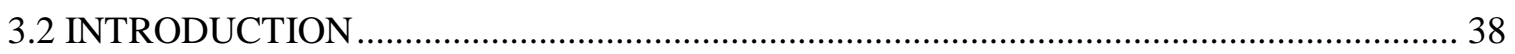

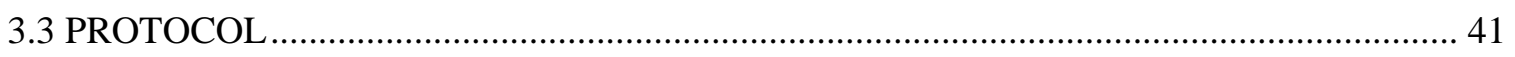

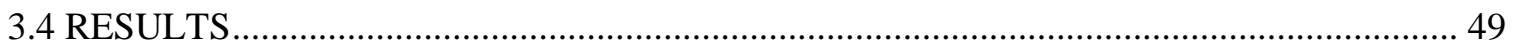

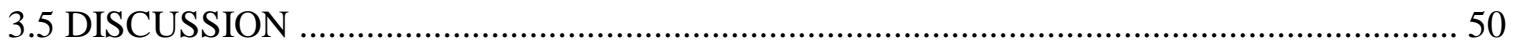

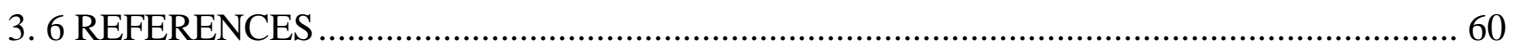

CHAPTER 4: SPATIAL EFFECTS OF NASONOV PHEROMONE AND ITS

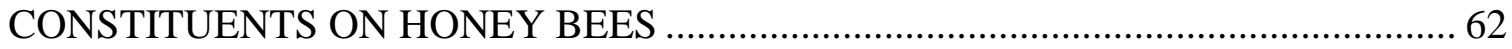

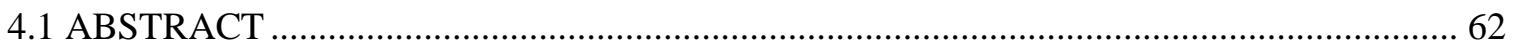

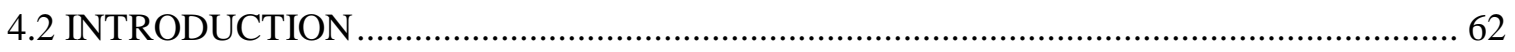

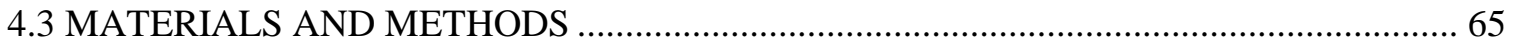




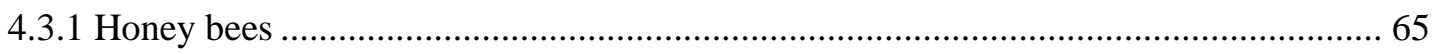

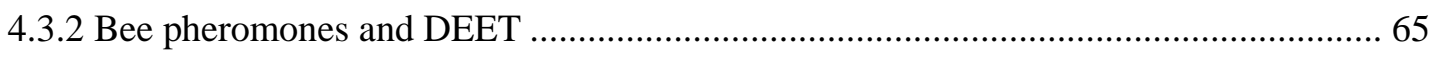

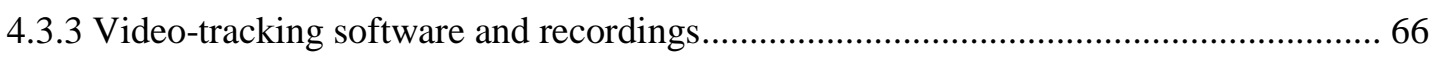

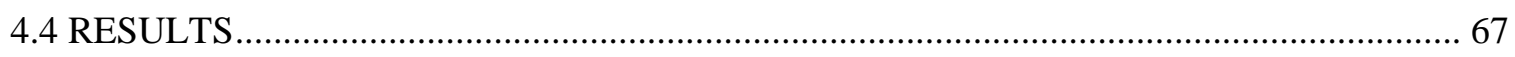

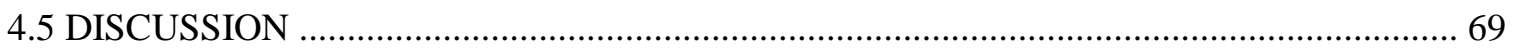

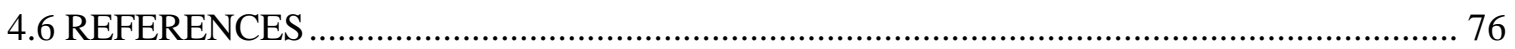

CHAPTER 5: EFFECT OF HETEROCYCLIC AMINES ON THE FEEDING

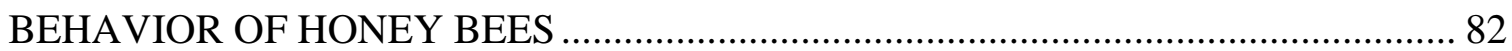

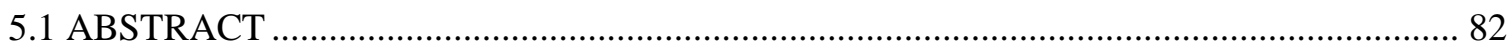

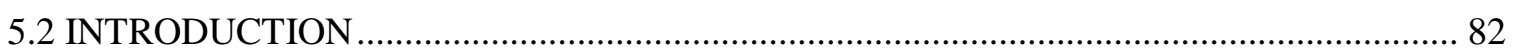

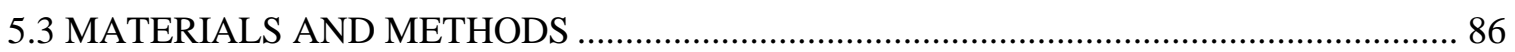

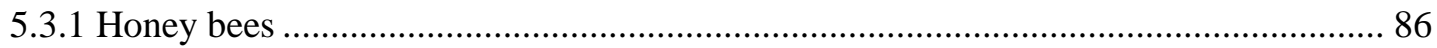

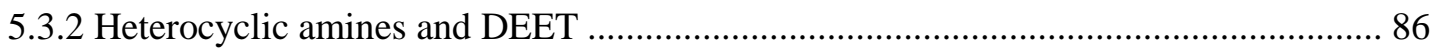

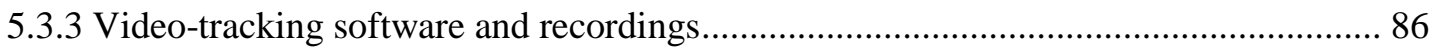

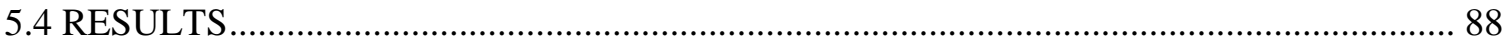

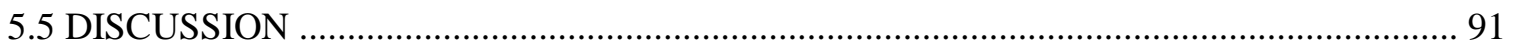

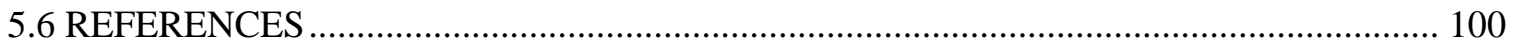

CHAPTER 6: EFFECT OF PIPERIDINE ON THE FORAGING BEHAVIOR OF

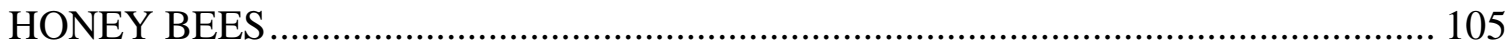

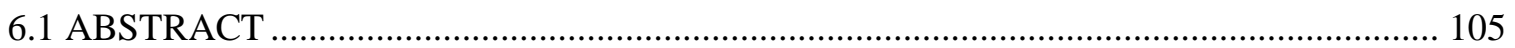

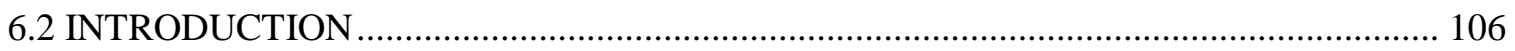

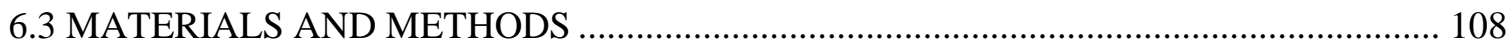

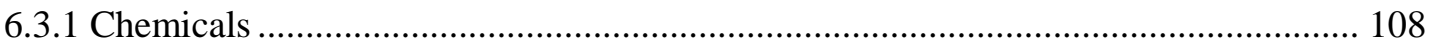

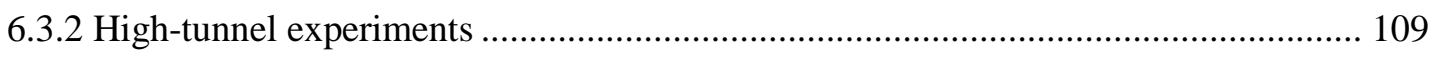

6.3.3 Field experiments with melon flowers and knapweed ........................................... 110

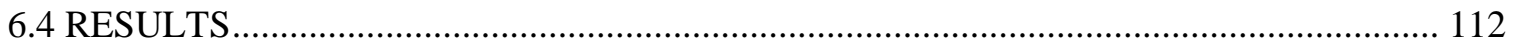

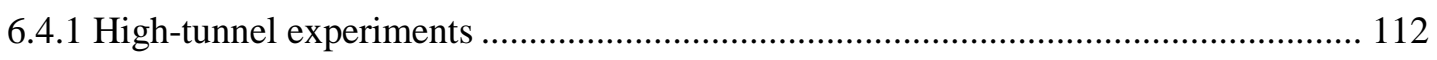

6.4.2 Field experiments with melon flowers and knapweed ........................................... 112

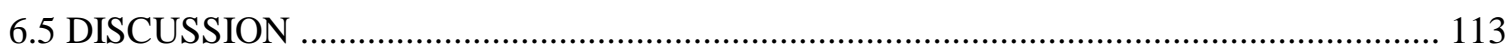

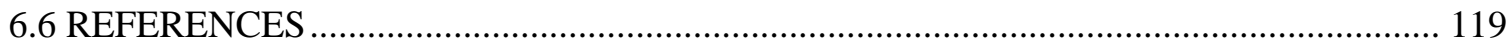

CHAPTER 7: ELECTROANTENNOGRAM RESPONSES OF HONEY BEE ANTENNAE TO PHEROMONAL AND HETEROCYCLIC AMINE COMPOUNDS

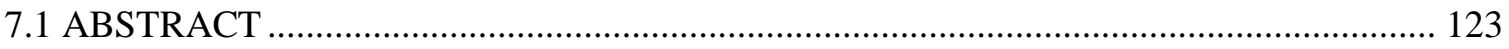

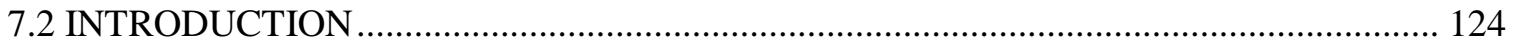

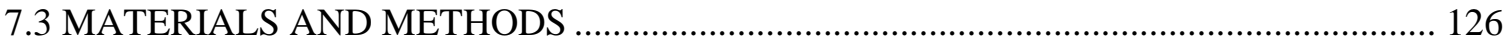




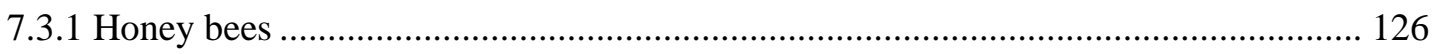

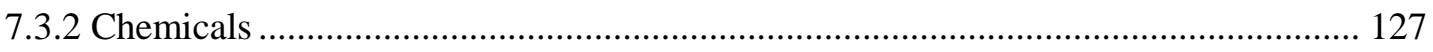

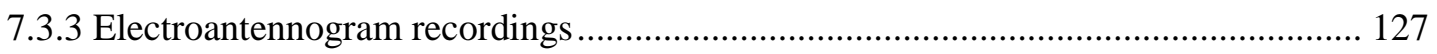

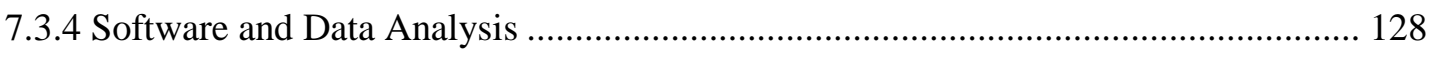

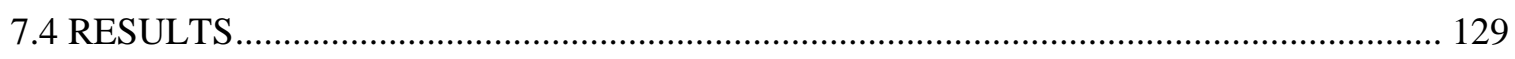

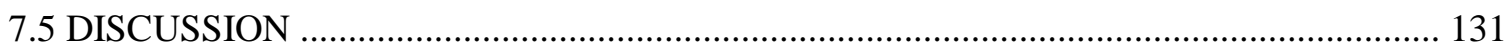

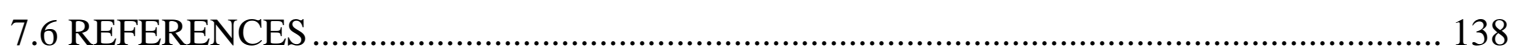

CHAPTER 8: CONCLUSIONS AND SUMMARY .................................................. 144

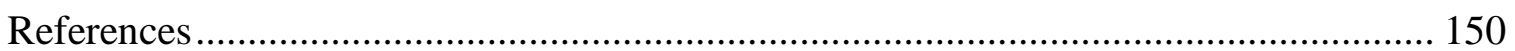




\section{INTRODUCTION}

The European honey bee, Apis mellifera L., generates hundreds of billions of dollars worldwide in honey production and pollination services (Gallai et al. 2009). In the United States and Europe, managed bee colony numbers have been declining as a result of multiple, interacting health stressors, including pesticide exposures (Aizen and Harder 2009, vanEngelsdorp and Meixner 2010, Smith et al. 2013). Bees encounter pesticides in a variety of ways, and the two most direct pathways from beekeeper pest management and agricultural pest management (Johnson et al. 2010, vanEngelsdorp and Meixner 2010, Smith et al. 2013). Pesticides can have a plethora of detrimental effects on bees ranging from acute toxicity to sublethal effects (vanEngelsdorp and Meixner 2010). A recent risk assessment study reported 161 pesticide residues have been identified in managed hives across the world (Sanchez-Bayo and Goka 2014).

Within the risk assessment study it was shown that insecticides comprised $52 \%$ of the residues, followed by fungicides at 25\%, herbicides at 17\%, and acaricides at 6\% (SanchezBayo and Goka 2014). Coumaphos and tau-fluvalinate, the active ingredients of the miticides Checkmite $+{ }^{\circledR}$ and Apistan ${ }^{\circledR}$, are the two most commonly found pesticides within managed bee colonies (Mullin et al. 2010, Sanchez-Bayo and Goka 2014, Garcia et al. 2017). Exposure to these miticides can have negative effects associated with olfactory learning, immune gene suppression, and decreased locomotor activity in bees (Taylor et al. 1987, Teeters et al. 2012, Williamson and Wright 2013, Cizelj et al. 2016). Several pesticides in the neonicotinoid class negatively affect immunocompetence, gland and 
respiratory development in bees, as well as reducing the survivability of queens exposed to other stressors (Hatjina et al. 2013, Brandt et al. 2016, Chaimanee et al. 2016, Christen et al. 2016, Dussaubat et al. 2016). Methods to reduce the interaction times of pollinators and pesticides are needed to help in reducing the stress on managed bee colonies. One method that warrants investigation is the use of repellents to keep foraging bees away from pesticide treated crops (Atkins et al. 1975, Free et al. 1985).

The focus of insect repellent discovery has primarily been in the area of personal protectants for biting arthropods to prevent disease transmission (Katz et al. 2008). Significant strides in long-lasting repellent development did not occur until World War II when the majority of military personnel were being exposed to disease endemic areas during combat (Moore and Debboun 2007). During this time DEET was discovered, and has since become the standard for arthropod repellent screens (Moore and Debboun 2007, Lawrence et al. 2014). However DEET's safety has been repeatedly questioned over the last 60 years, which has led to the investigations of alternative safer repellents (Diaz 2016). Several hundred compounds related to piperidine were subsequently screened for repellent attributes (Moore and Debboun 2007).

Analogs of piperidine have been shown to be highly effective in repelling ticks, mosquitoes, and chigger mites (Schreck et al. 1995, Solberg et al. 1995, Klun et al. 2001). Piperidine itself is a six-membered ring structure of five carbons and one nitrogen. It, along with several other heterocyclic amines, have been shown to repel mosquitoes from hosts by inducing hyposmia or anosmia, which ultimately inhibits olfaction by the mosquitoes 
(Bernier et al. 2012). The piperidine analog, picaridin, is recognized by the Environmental Protection Agency as a topical repellent along with several other compounds including a couple of plant-derived oils, citronella and catnip ("United States Environmental Protection Agency" 2017).

Prior to the development of the long-lasting repellents, essential oils were utilized; however, due to their volatility, the effects were of limited duration (Moore and Debboun 2007). Citronella and catnip have both been shown to repel mosquitoes and catnip has been found to be a better spatial repellent than DEET (Tawatsin et al. 2001, Bernier et al. 2005, Carroll and Loye 2006). Interestingly, pheromonal compounds within bees have been found to have repellent effects in bees and other organisms. The mandibular pheromone 2-heptanone has been shown to repel foragers from shrubs sprayed with it (Vallet et al. 1991). Geraniol, a component of the bee orienting pheromone Nasonov pheromone, has been found to be repellent to the parasitic mite Varroa jacobsoni (Hoppe and Ritter 1988). Additionally, the honey bee alarm pheromone isoamyl acetate has been shown to repel foragers from crops sprayed with it (Free et al. 1985).

However, an effective repellent for honey bees remains to be developed, and thus the natural compounds and heterocyclic amine chemistries provide a unique opportunity for the investigation into their repellent activity in honey bees. The focus of the proposed research was to test the hypothesis that bee pheromonal compounds and the heterocyclic amines induce repellent behavior, using a laboratory video tracking assay, semi-field and 
field assays, and electrophysiology techniques. This study examined the repellent efficacy of these compounds as well as the mode of detection utilized by the bees. 


\section{CHAPTER 1}

\section{LITERATURE REVIEW}

\subsection{Pollinator Significance}

The European honey bee, Apis mellifera L., was first domesticated by Egyptians before 2600 BCE. The practice was adopted by many cultures spreading through what became medieval Europe. Subsequently, European beekeepers spread beekeeping and the bees around the world. The original purpose of beekeeping was to provide honey as a sweetener (vanEngelsdorp and Meixner 2010). Today, honey is an international commodity with estimated global production values in the billions of dollars (http://faostat.fao.org/). However, it is the pollination services that managed honey bee colonies provide that make beekeeping necessary for modern day agriculture (vanEngelsdorp and Meixner 2010). Pollination plays a significant role in agriculture by increasing crop yield, seed production, and the quality of crops that humans and animals rely on for food. These provide improved nutritional benefits as well as the ability to improve the development of new crop varieties (Klein et al. 2007, Klatt et al. 2014). Evidence has shown that honey bees are especially important to certain monocultures and without their pollination services, crop yields can decrease by $90 \%$ (McGregor 1976, Southwick and Southwick 1992, Delaplane and Mayer 2000). Additionally, it has been observed that pollinator declines parallel reduced abundance of plant species that require pollinator services (Biesmeijer et al. 2006). The major global crops (cereals and sugarcane) are wind- or self-pollinated; however, there are several important fruits that are pollinated by animals. Animal pollination is primarily 
performed by insects, although there are a few commodity crops that are pollinated by vertebrates. Single crops that are pollinated by animals including insects represent $c a$. $35 \%$ of the global food production (Klein et al. 2007). The economic value of pollination is globally valued at more than $\$ 200$ billion USD (Gallai et al. 2009). The value of pollination in agriculture for the United States and Canada has been estimated to be more than $\$ 20$ billion USD. In the United States alone, the economic value of pollination is estimated to be $\$ 15$ billion USD (Calderone 2012). It is clear that pollination services are both economically and agriculturally important; however, high annual losses of managed honey bee colonies threaten the apiculture industry's ability to keep up with the increasing demand of pollination services. (vanEngelsdorp and Meixner 2010, Steinhauer et al. 2014, Lee, Steinhauer, Rennich, Wilson, Tarpy, Caron, Rose, Delaplane, Baylis, Lengerich, Pettis, Skinner, Wilkes, Sagili, and vanEngelsdorp 2015, Lee, Steinhauer, Rennich, Wilson, Tarpy, Caron, Rose, Delaplane, Baylis, Lengerich, Pettis, Skinner, Wilkes, Sagili, and VanEngelsdorp 2015, Seitz et al. 2016).

\subsection{Honey Bee Decline}

The global number of managed honey bee colonies has increased by approximately $45 \%$ since 1961. However, the United States and many European countries have experienced significant declines in managed honey bee colonies. From 1947-2008, the United States lost $61 \%$ of managed honey bee colonies dropping from 5.9 to 2.3 million, while Europe lost an estimated 27\% of their colonies from 1961-2007 (Aizen and Harder 2009, vanEngelsdorp and Meixner 2010). US beekeepers have attempted to mitigate this loss, and the number of managed bee colonies has seen a small increase back to 2.7 million 
honey producing colonies since 2015 (Seitz et al. 2016, USDA-NASS 2017). The European Union (EU) does not have an annual census comparable to the USDA National Agriculture Statistics Service and, thus, there is limited survival data for honey bee colonies in Europe. During 2012-2014, a study was undertaken to report on the annual colony losses from $17 \mathrm{EU}$ countries. The authors found that colony loss was highly variable across the 17 countries surveyed. Over the two-year study period, annual losses ranged from 2.6$36 \%$ and it was observed that high summer loss were associated with high winter loss (Laurent et al. 2016, Jacques et al. 2017). In 2014-2015, Seitz et al. (2016) reported annual honey bee mortality rates to range from $14-63 \%$ across the United States. Currently, beekeepers lose approximately 50\% of their colonies each year in the United States. These losses exceed the $19 \%$ that surveyed beekeepers deemed as "acceptable" in 2015 . To recover from these losses, beekeepers are required to split surviving colonies or purchase new colonies, which significantly increases their operational costs (Seitz et al. 2016). There is no single factor that can be linked to these annual losses, but rather multiple factors interacting with each other resulting in colony mortality (Potts et al. 2010, vanEngelsdorp and Meixner 2010). The most common factors reported by beekeepers in the United States are queen failure, starvation, Varroa destructor mite infestation, and weak overwintering colonies (Seitz et al. 2016). The varroa mite is a primary driver for several transmittable diseases in honey bees, including deformed wing virus, acute bee paralysis virus, Kashmir bee virus, and the Israeli acute paralysis virus. High varroa mite infestations and viral outbreaks can significantly increase colony mortality (Siede et al. 2008). There are other disease causing organisms that threaten honey bee health including the microsporidian Nosema ceranae, which has been reported to cause energetic stress and reduce the lifespan 
of infected honey bees (Mayack and Naug 2009). The bacterium Paenibacillus larvae, the causative agent for American foulbrood disease, has been associated with reduced productivity and economic losses of honey bee colonies valued in the millions of dollars (Eischen et al. 2005, vanEngelsdorp and Meixner 2010). Other stressors that should be taken into consideration include beekeeping practices, genetic variability of the colonies, weak queens, weather and climate, adequate bee forage, and pesticides (vanEngelsdorp and Meixner 2010).

\subsection{Pesticide Exposure}

Honey bee colony loss resulting from pesticide exposures is a highly debated topic due to the economic benefit pesticides have in agriculture, while at the same time evidence shows that they are detrimental to honey bee health (Goulson et al. 2015). Honey bees encounter pesticides in several ways. The two most direct pathways are from the crops that they forage on and from within the hive. Pesticide contact occurring outside of the hive is due to crop protection practices, while in-hive contact is due to beekeeper use of chemical control for the parasitic mites, bacteria, and microsporidia that infest bees (Johnson et al. 2010, vanEngelsdorp and Meixner 2010, Smith et al. 2013). When foraging, adult bees can pick up pesticides from various sources including nectar, pollen, guttation fluid, soil, residues on leaves from foliar sprays and dusts from treated seeds. These residues and forage are brought back to the hive and the remainder of the colony can be exposed through the processed nectar and pollen (Thompson 2010, Cresswell 2011, Fairbrother et al. 2014). 
There are a vast number of pesticides that are potential threats to honey bee health. Pesticide effects that have been seen in honey bees can range from acute toxicity to sublethal effects, which include paralysis, disorientation, and behavioral changes (vanEngelsdorp and Meixner 2010). Mullin et al. (2010) found 121 different pesticide residues within 887 hive samples received from 23 different states in the US and one province in Canada. The types of pesticides found within the hive samples included pyrethroids (13\%), organophosphates (13\%), carbamates (7\%), neonicotinoids (5\%), chlorinated cyclodienes (5\%), organochlorines (4\%), insect growth regulators $(3 \%)$, formamidines (2\%), several miticides (7\%), synergists $(2 \%)$, fungicides $(25 \%)$, and herbicides (14\%). A recent risk assessment by Sanchez-Bayo and Goka (2014) found that 161 pesticide residues have been identified in bee hives across the world. Insecticides comprised $52 \%$ of the residues. Fungicides were the second largest group found at 25\%, followed by herbicides at $17 \%$, and finally acaricides at $6 \%$. Analyses performed on the food stores of colonies found that 124 compounds were in pollen while 77 were found in honey (Sanchez-Bayo and Goka 2014).

Foraging workers are exposed to varying concentrations of pesticides as they must carry pollen and nectar back to the hive (Sanchez-Bayo and Goka 2014, Silvina et al. 2017). Interestingly, Silvina et al (2017) found that the amount of insecticide that is returned to a hive varies by compound. They suggested that it is possibly due to differences in volatility of compounds, with bees detecting higher volatile compounds and avoiding flowers sprayed with those compounds. However, this factor does not seem to provide enough deterrence to reduce the amount of pesticide residues found within bee food stores. 
The two most common pesticides found in bee colonies are coumaphos and tau-fluvalinate. These are the active ingredients within Checkmite $+{ }^{\circledR}$ and Apistan ${ }^{\circledR}$, the miticides used to control Varroa mite (Mullin et al. 2010, Sanchez-Bayo and Goka 2014, Garcia et al. 2017). tau-Fluvalinate is a pyrethroid class miticide that binds to voltage-gated sodium channels prolonging their inactivation, which eventually results in the loss of nerve excitability (Yu 2008). This class can be highly toxic to bees; however, cytochrome P450 monooxygenases are reported to metabolically detoxify tau-fluvalinate and reduce its toxicity to bees (Johnson et al. 2006). Coumaphos was registered in the U.S. in 1999 after tau-fluvalinate efficacy started to decrease due to Varroa mite development of resistance (Lodesani et al. 1995, Elzen et al. 1999, Johnson et al. 2010). It is an organophosphate class miticide that is bioactivated by cytochrome P450 enzymes into the more toxic metabolite coumaphos oxon. It then binds to and inhibits acetylcholinesterase resulting in hyperexcitation of nerve cells (Yu 2008). Both pesticides are highly lipophilic and can be absorbed into bee hive components such as wax (Bogdanov et al. 1997, Johnson et al. 2010, Mullin et al. 2010). Interactions between these two have been shown to have synergistic effects on each other increasing honey bee mortality when both are found within a colony (Johnson et al. 2009). Another class of pesticides that has come under increased scrutiny recently are the neonicotinoids. The most widely used insecticides in the world (Goulson 2013).

A recent study found that neonicotinoids were the most common pesticides studied in association with honey bees, ahead of coumaphos and tau-fluvalinate, over the last decade (Benuszak et al. 2017). Tison et al. (2016) found that sublethal exposures to the 
neonicotinoid thiacloprid had detrimental effects on honey bee navigation, motivation to forage, and social communication. Other studies have shown that neonicotinoids decrease the immunocompetence of honey bees increasing disease susceptibility (Brandt et al. 2016, Christen et al. 2016). Adult bee exposure to imidacloprid, during the first two weeks after emergence, negatively affects development of the hypopharyngeal glands and respiratory system (Hatjina et al. 2013). Imidacloprid has also been shown to decrease the survival of queen honey bees that have Nosema ceranae infections (Dussaubat et al. 2016). Additionally, it has been shown to reduce sperm viability in queens ultimately affecting the long term success of a colony (Chaimanee et al. 2016). The European Union became so concerned about the effects seen with neonicotinoids on pollinators that they placed a temporary ban on their use in 2013 to determine whether this class is having a significant effect on honey bee colony loss (Fairbrother et al. 2014, Benuszak et al. 2017).

It is likely that pesticides will continue to be used in the future, as achieving high yields in modern agriculture requires their use (vanEngelsdorp and Meixner 2010). In order to mitigate honey bee exposure to harmful compounds, new regulations and methods need to be developed and implemented to protect the agricultural benefit of pollinators (Mullin et al. 2010). One potential method, is to utilize repellents during pesticide spraying to keep pollinators away from the treated crops (Callbreath 1900, Atkins et al. 1975, Free et al. 1985). However, before discussing repellents, insect olfaction will be reviewed.

\subsection{Insect Olfaction}


Insect odorant detection begins at the antennae and requires a number of olfactory proteins, including odorant binding proteins (OBPs), odorant degrading enzymes (ODEs), odorant receptors (ORs), ionotropic receptors (IRs), and sensory neuron membrane proteins (SNMPs) (Leal 2013). ORs are the most well understood receptor proteins (Andersson et al. 2015). Odorants enter through pores located on the antennae and are picked up by OBPs. Odorants are transported through the sensillar lymph to an OR that is located on an odorant receptor neuron (ORN) (Leal 2005). The odorant-OBP complex activates the OR through two hypothesized modes of action. The first model, the odorant-OBP complex directly activates the OR (Laughlin et al. 2008), whereas, in the second model the complex releases the bound odorant that activates the OR. Additional odorant molecules are broken down via degradation through ODEs or collection by a molecular trap (Leal 2013). Once the ORN has been activated, action potentials travel down the axon to the antennal lobe where they terminate into glomeruli (Hildebrand and Shepherd 1997). Then, projection neurons transmit the stimulus to higher brain centers, such as the mushroom body and lateral protocerebrum, which results in a response to the odorant (Riffel and Hildebrand 2016). The types of receptors that are expressed on the ORNs have significant effects on the olfactory system, and adding receptors to the ORNs can elicit behavioral responses that are identical to the activation of the native receptors (Sakurai et al. 2011, Leal 2013).

The insect olfactory system is highly sensitive and specific for pheromonal odorants as well as odorants released by plants (Hansson et al. 1999, Larsson and Leal 1999, Nikonov et al. 2002). Honey bees use their olfactory system to locate flowers and for social communication. The honey bee genome contains $170 \mathrm{OR}$ genes compared to the 62 and 
72 OR genes in Drosophila melanogaster and Anopheles gambiae, respectively (Robertson and Wanner 2006). In contrast, the number of gustatory receptor genes (GRs) that the honey bee expresses 10 (Dunipace et al. 2001, Scott et al. 2001, Robertson and Wanner 2006) is significantly lower than the GRs expressed in D. melanogaster 68 (Robertson et al. 2003) and An. gambiae 76 (Hill et al. 2002). The disparity between the species and the quantity of the receptors may be due to the co-evolution of honey bees with the plants they forage on. Plants evolved mechanisms to attract bees for pollination services and, in return, the bees receive nectar and pollen used for food production in the hive. Within the hive the adult bees feed the larval bees, resulting in the larval bees not requiring mechanisms to locate food, nor detect defensive compounds within their food sources. Bees antennate objects that they encounter, including kin, as a form of chemoreception. It has been hypothesized that, due to the chemoreception function of the antennae, some of the ORs found within the antennae are functionally similar to contact gustatory receptors and, therefore, there is no need for those GRs (Robertson and Wanner 2006).

While ORs are necessary for odorant detection, OBPs are also a key component of the detection system and increase sensitivity of the olfactory system (Leal 2013). The OBPs play several roles within the odorant detection system. They solubilize ligands, assist in transport of hydrophobic molecules through the sensillar lymph, and affect the sensitivity of the sensory system (Leal 2013). Using electroantennogram techniques, it was reported that reducing the transcription of one major OBP gene in Culex quinquefasciatus can significantly decrease the response of the mosquito to known attractants (Pelletier et al. 2010). There are 21 genes encoding OBPs in the honey bee genome; whereas, the $A n$. 
gambiae and D. melanogaster genomes have over 70 and 50 OBP encoding genes, respectively. There are 9 out of 21 honey bee OBP genes found in the antennae, with the remaining OBP genes found in other tissues during development (Forêt and Maleszka 2006). The honey bee antennal OBPs were renamed and described as antennal specific proteins (ASPs) (Briand et al. 2000, Dani et al. 2010). ASP1 is reported to bind to queen pheromone, ASP2 binds plant odors and 2-heptanone, and ASP3c bind to brood pheromone components (Briand et al. 2002).

\subsection{Insect Repellents}

The definition of "repellent" has been a topic of debate. Dethier (1947) broadly defines a repellent to be a source that causes an avoidant reaction whereas Kennedy (1947) describes repellency as a source that affects the distribution of insects. Dethier et al. (1960) later provide a stricter definition of repellent as a chemical that elicits directed movement away from a source. However, this definition does not allow for the inclusion of sources that potentially mask detection capabilities of the hosts. Barton-Browne (1977) state that repellents are typically tested against chemical attractants and that they are rated based upon their ability to suppress an insects response to the attractants. Bernier et al. (2007) later describe a repellent as a chemical that prevents an insect from reaching a target that it would otherwise be attracted to if the chemical was not present (Bernier et al. 2007). There are two types of repellents depending on their mode of action. Spatial repellents work by repelling at a distance, while contact repellents require an insect to physically contact a chemical to elicit a repellent action (Dethier 1947, Gouck et al. 1967, Bissinger and Roe 2014). Repellent chemicals can potentially have both spatial or contact activity (Bissinger 
and Roe 2010). DEET has been shown to act as a better contact repellent at times and, at other times, it has been shown to be an effective spatial repellent (Khan and Maibach 1972, Bernier et al. 2005). The volatility and concentration of a chemical in its vapor phase can dictate whether the repellent has spatial or contact activity. Chemicals with high volatility will have a higher vapor phase concentration, which will be detectable at a distance. Low volatile chemicals have repellent activity when an insect contacts or is in close proximity to the chemical (Bernier et al. 2007).

Historically, insect repellents (IRs) have been used to prevent the spread of deadly diseases by arthropod vectors (Katz et al. 2008). Egyptians (484 BCE - 425 BCE) may have been the earliest to record the use of a repellent to keep mosquitoes away while they slept and fished (Charlwood 2003). Additionally, many traditional methods have been reported to be effective in reducing the landings and densities of biting arthropods (Moore and Debboun 2007). Many of these methods require burning plant material to release volatiles and create smoke that results in repellent activity (Silva 1991, Dulhunty et al. 2000). While effective in repelling arthropods, these methods potentially are hazardous to human health and, thus, new methods were needed (Smith and Mehta 2003, Moore and Debboun 2007). The development of long-lasting repellents did not begin until World War II when military troops had to fight in disease endemic areas. Prior personal repellents, such as essential oils, had limited duration (Moore and Debboun 2007). Dimethyl phthalate and Indalone ${ }^{\circledR}$ were reported to be effective repellents for protection against chigger mites (Madden et al. 1944). The popularity of these compounds was short-lived following the discovery of DEET (N,N-diethyl-3-methylbenzamide) (Moore and Debboun 2007). 
DEET is the most widely used and most efficacious IR to be developed in the past 60 years (Katz et al. 2008, Dickens and Bohbot 2013). Before World War II, and the introduction of DEET, four IRs were available for consumer use, including the oil of citronella, dialkyl phthalate, Indalone ${ }^{\circledR}$, and Rutgers 612 (Brown and Hebert 1997). A number of attributes are desired for a successful IR, such as (1) lasting effect upon a variety of arthropods; (2) non-irritating to the user when applied to skin or clothing; (3) odorless or pleasant odor; (4) no effect on clothing; (5) no oily appearance when applied to skin and able to withstand sweating, washing, and wiping by the user; (6) no effect upon commonly used plastics; and (7) chemical stability and affordability for widespread use (Brown and Hebert 1997). DEET has become an important IR and is considered to be the gold standard for topically applied arthropod repellent testing. It is recommended by the World Health Organization (WHO) and the United States Environmental Protection Agency (EPA) as a positive control for novel repellent screening (Moore and Debboun 2007, Lawrence et al. 2014). DEET has been successfully used for more than 60 years. However, due to concerns over long-term daily use the safety of DEET has been highly debated. This has led to the discovery of new and safer repellents (Fradin 1998, Diaz 2016).

The discovery of DEET has led to the study of several repellents. DEPA ( $N, N$-diethyl phenyl acetamide) was developed around the same time as DEET (McCabe et al. 1954). Two analogs of it were found to repel two species of mosquito better than DEET while also possessing low toxicity (Debboun and Wagman 2004). In 1976, Merk developed insect repellent 3535 (IR 3535) (Klier and Kuhlow 1976), with several studies reporting 
variable effects of IR 3535 compared to DEET (Rettich 1999, Barnard et al. 2002, Cilek et al. 2004). However, there are no reports of toxicity or adverse effects associated with IR 3535 (Moore and Debboun 2007). During the 1970s, hundreds of synthetic compounds related to piperidine were developed, and are currently being re-evaluated for their potential use as repellents (Moore and Debboun 2007).

Piperidine is a six-membered ring structure with five carbons and one nitrogen. It was isolated from black pepper in 1852 (Warnhoff 1998). Piperidine analogs have been found to repel several types of arthropods. The analog 1-(3-cyclohexen-1-ylcarbonyl)-2methylpiperidine was shown to repel the lone star tick, Amblyomma americanum, away from humans significantly longer than DEET and one of its stereoisomers has been reported as an effective repellent for mosquitoes and chigger mites (Solberg et al. 1995, Klun et al. 2001). Schreck et al. (1995) found that 1-(3-cyclohexenyl-carbonyl) piperidine provided a longer protection time against the lone star tick compared to DEET. The EPA currently recognizes seven active ingredients for topically applied insect repellents, including Picaridin, a piperidine compound shown to be a highly effective tick repellent (Carroll et al. 2008, "United States Environmental Protection Agency” 2017). Synthetic repellents are not the only option for personal protection against biting arthropods. The EPA also recognizes the two plant-derived oils (citronella and catnip) for topical application and protection against arthropod pests, ("United States Environmental Protection Agency" 2017). 
There are several essential oils reported to repel biting arthropods; however, these oils only provide short-term protection (Moore and Debboun 2007). Citronella oil is no exception due to its high volatility. This essential oil is effective at deterring mosquitoes for up to two hours, and is ineffective on other biting arthropods (Tawatsin et al. 2001, Carroll and Loye 2006). Catnip oil was shown to reduce the mean attraction level of mosquitoes, to an attractive blend in olfactometer bioassays, by $80 \%$ compared to DEET, which reduced the level by only $25 \%$ (Bernier et al. 2005). This indicates that catnip oil is a higher efficacy spatial repellent compared to DEET (Bernier et al. 2005).

Honey bees have a repertoire of pheromones that elicit repellent or attractive effects. 2Heptanone is a worker mandibular pheromone that is reported to be an alarm pheromone and can act as a repellent to honey bees visiting shrubs treated with the pheromone (Vallet et al. 1991). Another pheromone of interest is the alarm pheromone isoamyl acetate (Boch et al. 1962). Free et al. (1985) showed that isoamyl acetate, as well as 2-heptanone, repelled honey bee foragers when applied to oil-seed rape, field beans, and sunflower heads. Conversely, there are pheromones that are used as attractants as well. Nasonov is a pheromone that is comprised of six monoterpenes and $(E, E)$ - farnesol, with the main constituents geraniol, nerol, and citral (Boch and Shearer 1964, Shearer and Boch 1966, Free et al. 1985). It is regarded as a primary tool to attract honey bee swarms. Schmidt (1999) reported that Nasonov pheromone attracted five times as many swarms than control treatments or other tested compounds. The Nasonov pheromone constituents, geraniol and citral, have been found to reduce feeding and cause mortality in the freshwater snail, Planobis corneus by inducing octopamine-like responses in major ganglia associated with 
feeding (Price and Berry 2008). Octopamine is a biogenic monoamine that acts as a neurotransmitter in invertebrates (Roeder 1999) however, octopamine receptor antagonists were unable to block the effects of the oils suggesting that the oils do not activate or inhibit octopamine receptors (Price and Berry 2008). Hoppe and Ritter (1988) report geraniol as a repellent for the parasitic mite Varroa jacobsoni whereas Ellis and Baxendale (1997) found that citral, applied as a fumigant, is toxic to the tracheal mite, Acarapis woodi (Rennie). Nerol is the geometric isomer of geraniol and they both elicit responses in different projection neurons of Manduca sexta (Reisenman et al. 2004). Nerol also elicits an alarm response in the stingless bee Trigona fulviventris (Guerin) (Johnson and Wiemer 1982). Finally, laboratory studies have shown increased aggressive behavior between bees raised in the presence of floral oils and those raised in the absence of them, providing evidence that floral scents are used as nest-mate recognition odors (Bowden et al. 1998).

There are several compounds reported to manipulate bee behavior, resulting in an avoidance effect from a sprayed object or location. While field testing has been the primary method for testing these compounds, the involved methods can be labor intensive and time consuming. The development of laboratory-based methods to evaluate chemical deterrents for bees would significantly increase the rate of testing different chemistries. An evaluation of compounds that historically have been used to repel biting arthropods provides a standard approach to test known chemical deterrents against bees. In turn, the successful discovery of an effective chemical(s) that manipulate bee behavior and deter bees from a pesticide-treated area provides an alternative approach for protecting these important pollinators. 


\section{CHAPTER 2: EFFECTS OF HONEY BEE PHEROMONAL COMPOUNDS ON THE MORTALITY AND FEEDING BEHAVIOR OF HONEY BEES}

\subsection{ABSTRACT}

The economic value of the honey bee, Apis mellifera L., necessitates the development of protection practices that reduce the stress of pesticide exposure to them. Utilizing a repellent to mitigate interaction times between bees and crops sprayed with pesticides is one potential method to reduce this stress. The aim of this study was to evaluate the toxicity and behavioral effects of honey bee pheromonal compounds. This was achieved using a glass tube assay that utilized honey weigh boats placed atop a sized filter paper treated with a test compound. Results show that synthetic Nasonov pheromone, an aggregation and queen locating pheromone, caused mortality within honey bees twice as fast (LT $\mathrm{LT}_{50} 1-2 \mathrm{hr}$ ) as its constituents citral, nerol, and geraniol (LT T0s $_{3} 3-5 \mathrm{hr}$ ). Alarm pheromones isoamyl acetate and 2-heptanone were found to have insignificant effects on mortality $(<30 \%$ at the conclusion of the tests). Interestingly, each of the pheromonal compounds tested reduced consumption of honey from the honey weigh boats. Nasonov and its constituents were as effective as DEET (repellent standard) in reducing feeding behavior completely, while the two alarm pheromones reduced feeding by $47-80 \%$. This potentially indicates a repellent effect generated by the pheromonal compounds to bees in the described laboratory assay.

\subsection{INTRODUCTION}

The European honey bee, Apis mellifera L., is an economic and agriculturally important insect that annually generates ca. $\$ 3.2$ billion worth of honey (http://faostat.fao.org/) and provides pollination services valued at more than $\$ 200$ billion USD, globally (Gallai et al. 
2009). The global population of managed honey bee colonies has increased by approximately $45 \%$ since 1961 . However, for the past 60 years, the United States and many European countries have experienced significant declines in bee colonies. From 1947-2008, the United States lost $61 \%$ of bee colonies reducing colony numbers from 5.9 to 2.3 million, while Europe lost an estimated $275 \%$ of their bee colonies from 1961-2007 (Aizen and Harder 2009, vanEngelsdorp and Meixner 2010). In the United States, beekeepers lose an average of 50\% of their managed colonies each year. These losses are well above the $19 \%$ that surveyed beekeepers deemed as "acceptable" in 2015 . Recovery of these losses requires beekeepers to make splits from surviving colonies or purchase new colonies, both of which significantly increase their operational costs (Seitz et al. 2016). There are a number of factors that contribute to colony loss (Potts et al. 2010, vanEngelsdorp and Meixner 2010). The most common factors that have been reported by beekeepers in the United States are queen failure, starvation, varroa mite infestation, and weak colonies going into winter (Seitz et al. 2016). Additional stressors that should be taken into consideration include beekeeping practices, genetic variability of the colonies, poor or weak queens, weather and climate, adequate bee forage, and pesticides (vanEngelsdorp and Meixner 2010).

Bees encounter pesticides in several ways. The two most direct pathways are from the crops on which they forage and from within the hive. Pesticide contact occurs from pest management strategies used by beekeepers to control in-hive pests and from farmers controlling agricultural pests (Johnson et al. 2010, vanEngelsdorp and Meixner 2010, Smith et al. 2013). When foraging, adult bees pick up pesticide residues from treated plants 
and return to the hive while bringing the collected forage. The remainder of the colony can then be exposed to the residues through the processed nectar and pollen (Thompson 2010, Cresswell 2011, Fairbrother et al. 2014). A recent risk assessment identified 161 different pesticide residues within hives across the world, which consisted of insecticides (52\%), fungicides (25\%), herbicides (17\%), and acaricides (6\%) (Sanchez-Bayo and Goka 2014).

Pesticides can have several different effects on bees ranging from acute toxicity to sublethal effects (vanEngelsdorp and Meixner 2010). Studies have shown that pesticide exposures can cause reduced motor function, reduced immunocompetence, impairment of olfactory learning, the reduction of antioxidants, as well as negative effects on navigation and social communication (Taylor et al. 1987, Haarmann et al. 2002, Teeters et al. 2012, Williamson and Wright 2013, Tison et al. 2016, Williams 2016). There is an urgent need to mitigate bee exposure to pesticide sprays and residues (Mullin et al. 2010). A potential method suggested is the use of a chemical repellent within a pesticide mixture to keep bees away from treated crops (Callbreath 1900, Atkins et al. 1975, Free et al. 1985).

\subsection{MATERIALS AND METHODS}

\subsubsection{Honey bees}

Honey bees were maintained in hives at the Prices Fork Research Facility of Virginia Tech (Blacksburg, VA) using standard beekeeping practices. Worker bees were collected from frames located in the uppermost super of a colony to ensure that newly emerged and nurse bees were not collected in high quantities the evening before the bioassays. Bees were kept in an incubator set at $32{ }^{\circ} \mathrm{C}$ with a relative humidity of $c a .70 \%$ with access to a $25 \%$ sucrose-water (w/v) solution. 


\subsubsection{Chemicals}

Geraniol, nerol, citral, isoamyl acetate, 2-heptanone, and DEET (Fig. 1) were purchased from Sigma-Aldrich Chemical Co. (St. Louis, MO). Synthetic Nasonov pheromone was purchased from Dadant \& Sons Beekeeping Equipment (Chatham, VA).

\subsubsection{Toxicity Bioassays}

Bees were collected 1-d prior to each bioassay. Twenty individual bees were placed into separate containers and provided ad libitum $25 \%$ sucrose-water (w/v) solution overnight. The following day five weigh boats $(n=5)$ were filled with $1 \mathrm{~g}$ of honey and a wire screen was placed on top of each weigh boat. Filter papers $(5 \times 6 \mathrm{~cm})$ were impregnated with 400 $\mu \mathrm{l}$ of each compound at $8,20,60$, or $100 \%$ using acetone as a carrier solvent. Test compound treated papers along with control filter papers that were treated with $100 \%$ acetone were allowed to air dry for 5 minutes. The impregnated filter papers were each placed into one end of a glass tube arena $(n=5)$ measuring $60 \mathrm{~cm}$ in length with openings at each end measuring $10 \mathrm{~cm}$ in diameter. A weigh boat of honey was then placed on top of each of the impregnated filter papers (Fig. 2). Honey bees were anesthetized with $\mathrm{CO}_{2}$ and 10 individuals were placed into each of the glass containers. The containers were placed into an environmental chamber and the assay was run for 8 hours. Mortality was recorded at five time points 30, 60, 120, 240, and 480 minutes. The lethal time $\mathrm{LT}_{25}, \mathrm{LT}_{50}$, and $\mathrm{LT}_{75}$ were estimated using log-probit analysis in Polo Plus software (LeOra Software LLC El Cerrito, CA) to report compound efficacy. All concentrations were run in triplicate. 


\subsubsection{Feeding inhibition bioassays}

Honey bees were collected the day prior to screening and 20 individuals were placed into separate $32 \mathrm{oz}$ containers and fed a $25 \%$ sucrose solution overnight. The day of testing, weigh boats $(\mathrm{n}=18)$ were filled with honey equaling $\approx 1 \mathrm{~g}$, and a wire screen was placed on top of each boat. Squares of $5 \times 6 \mathrm{~cm}$ filter paper $(n=18)$ were treated with varying percentages of compound $(8,20,60$, and $100 \%)$ in acetone vehicle. The compound treated papers along with control filter papers, that had been were treated with $100 \%$ acetone, were allowed to air dry for 5 minutes. The treated filter papers were each placed into one end of a glass tube arena $(n=18)$ measuring $60 \mathrm{~cm}$ in length with openings at each end measuring $10 \mathrm{~cm}$ in diameter. A weigh boat of honey was then placed on top of each of the impregnated filter papers. Honey bees were anesthetized with $\mathrm{CO}_{2}$ and 20 individuals were placed into each of the glass containers. The containers were placed into an environmental chamber and the assay was run for 4 hours. Controls were run for 2 hours as mortality increased significantly for the tested compounds after this time point. At the conclusion of the assay, the weigh boats were removed from the glass containers and weighed again to determine the percentage of honey consumed over the 4 hours. All concentrations were run in triplicate. Data were analyzed using GraphPad Prism software (La Jolla, CA).

\subsection{RESULTS}

\subsubsection{Toxicity bioassays}


Nasonov pheromone was the most toxic compound at the 8,20 , and $60 \%$ concentrations with the $\mathrm{LT}_{50}$ ranging from 83-119 min compared to geraniol, citral, and nerol with $\mathrm{LT}_{50}$ ranges of 187-283 $\mathrm{min}, 225-309 \mathrm{~min}$, and 178-218 $\mathrm{min}$, respectively (Table 1). There were no significant differences in the toxicities of each compound at $100 \%$. There was a significant increase in bee mortality for those individuals exposed to geraniol, citral, and nerol for $2 \mathrm{~h}$; whereas, 2-heptanone, isoamyl acetate, and DEET were less toxic to the bees. Thirty percent mortality, or less, was observed with bees exposed to 2-heptanone, isoamyl acetate, and DEET for $8 \mathrm{~h}$ (data not shown).

\subsubsection{Feeding inhibition bioassays}

There was a significant increase in the feeding inhibition of bees exposed to Nasonov pheromone, geraniol, citral, nerol, 2-heptanone, and DEET for $4 \mathrm{hr}$ (Fig. 3). Bees exposed to the control treatment (acetone treated filter paper) consumed $0.38 \pm 0.05 \mathrm{~g}$ of honey in $2 \mathrm{hr}$ (Fig. 3A-G). There was little to no honey consumed by bees exposed to Nasonov pheromone, geraniol, citral, nerol, and DEET at 8-100\% (Fig. 3A-D, G). Bees exposed to 2-heptanone and isoamyl acetate at $8-100 \%$ were observed to consume ca. $74 \%$ and $50 \%$ less honey, respectively, compared to the bees in the control treatments (Fig. 3E-F).

\subsection{DISCUSSION}

The goal of this study was to develop a laboratory assay for screening compounds with repellent and attractant activity to bees. Nasonov pheromone was selected as a standard attractant in the glass tube assay, but it was later discovered that bees exposed to the compound in a closed arena were not attracted to the pheromone. In addition, Nasonov 
pheromone was not only acutely toxic to the bees, but was more toxic than the individual components geraniol, nerol, and citral at concentrations less than $100 \%$. This was an interesting finding, since Nasonov pheromone is a beekeeper-applied attractant for the collection of bee swarms in hive boxes (Schmidt 1999). Synthetic Nasonov pheromone is applied within a swarm box per manufacturer guidelines. The method typically involves proprietary slow release vials placed directly within a swarm box until a bee swarm accepts the box as a location to settle in. Additionally, it is probable that a mixture of geraniol, nerol, and citral (collectively Nasonov pheromone) may have a synergistic effect that increases the toxicity of the mixture compared to the individual components. Pesticides have been shown to have similar synergistic effects when administered simultaneously and, thereby, having higher toxicity compared to the individual parts (Keplinge and Deichman. 1967). The concentrations used within this study are also high compared to the Environmental Protection Agency's guidelines for acute toxicity in bees. The EPA recommends that pesticides have an $\mathrm{LD}_{50}$ value of less than $11 \mu \mathrm{g} / \mathrm{bee}$, which is $c a .110$ ppm (EPA 2016). The lowest concentration of compound used within this study was $8 \%$, converted to ppm is 80,000 ppm which is 727 times higher than the EPA guideline.

DEET, 2-heptanone, and isoamyl acetate were not acutely toxic to the bees, with less than $30 \%$ mortality observed at the tested concentrations. 2-Heptanone is reported to have low acute toxicity to the tomato fruit worm, Heliothis zea (Boddie) (Dimock et al. 1982), whereas DEET was reported to be acutely toxic to three mosquito species as well as house flies via topical treatments (Swale et al. 2014). Isoamyl acetate is reported to be acutely toxic to anaerobic bacteria (Playne and Smith 1983). 
Nasonov pheromone, geraniol, nerol, citral, 2-heptanone, isoamyl acetate, and DEET significantly inhibited the amount of honey consumed by the bees compared to the control treatments. Antifeedants are described as either eliciting repellent activity without the insect directly contacting the treated source (spatial) or eliciting feeding deterrence after the insect comes in contact with a treated surface (Koul et al. 2008). Geraniol and citral are reported to inhibit the feeding activity of snails (Price and Berry 2008), which is similar to the reduced feeding of bees exposed to the compounds. Nerol is a geometric isomer of geraniol, and was expected to inhibit the honey consumption of bees similar to that of geraniol. In addition, nerol is reported to be a highly effective spatial repellent for the German cockroach, Blattella germanica (Inazuka 1983). DEET is considered to be the most successful arthropod repellent (Katz et al. 2008, Dickens and Bohbot 2013) and isoamyl acetate along with 2-heptanone have been shown to repel foraging bees from oil seed rape and sunflower heads (Free et al. 1985). Thus, it was anticipated that DEET, isoamyl acetate, and 2-heptanone would act as repellents to reduce the honey consumption of bees. Dethier et al. (1960) strictly defines a repellent as a chemical that elicits a directed movement away from its source, thus further investigation is needed to determine if the behavior of the bees within the glass arenas follows this definition. However, Dethier's definition does not account for compounds that mask odorant detection by the host. In turn, Bernier et al. (2007) describe a repellent as a chemical that prevents an insect from reaching a target that it would otherwise be attracted to if the compound was not present. Based on this definition, and the observations of reduction in honey consumption, the compounds tested in this study did repel the bees away from a food source. Though additional studies 
are required to evaluate the movement and location of the bees to further validate that these compounds are preventing the bees from reaching the targeted food source. 

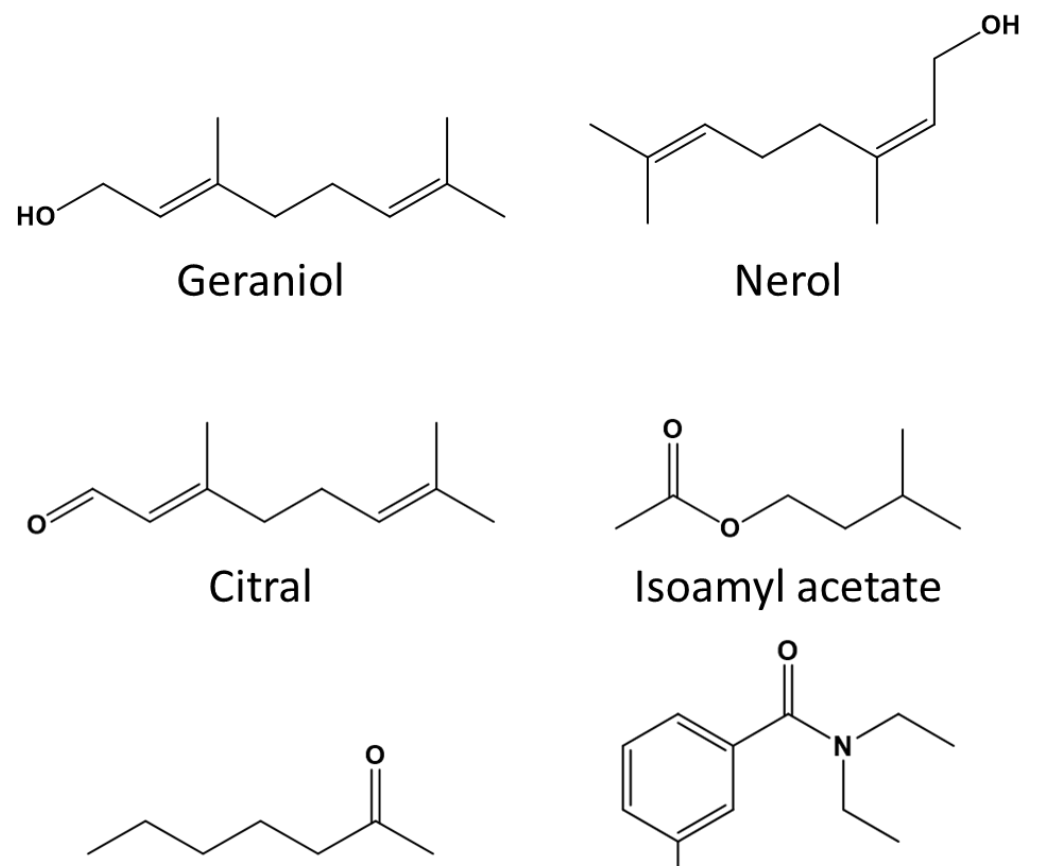

2-Heptanone

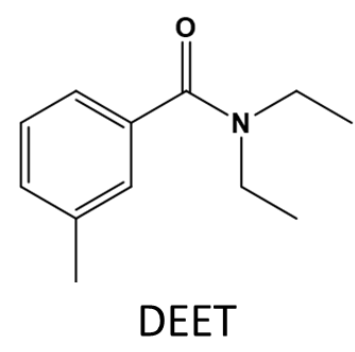

Figure 1. Chemical structures of the Nasonov pheromone components geraniol, nerol, and citral, the alarm pheromones isoamyl acetate and 2heptanone, and the arthropod repellent DEET ( $N, N$-diethyl-3methylbenzamide). 
Table 1. Toxicity of synthetic Nasonov pheromone, geraniol, citral and nerol to honey bees. Toxicity data are presented as $\mathrm{LT}_{25}, \mathrm{LT}_{50}, \mathrm{LT}_{75}$, and their $95 \%$ confidence intervals (CI) in minutes, or the time at which 25,50 , and $75 \%$ of the tested bees were dead, respectively in the bioassay. Each compound was impregnated onto a filter paper at the listed concentrations into $100 \%$ acetone (v/v). Each bioassay was replicated in triplicate. Log-probit analysis was used to estimate the endpoint times for each compound.

\begin{tabular}{|c|c|c|c|c|c|}
\hline Lethal Time & Concentration & Nasonov & Geraniol & Citral & Nerol \\
\hline \multirow[t]{4}{*}{$\begin{array}{l}\mathbf{L T}_{25} \\
(\mathrm{~min}, 95 \% \mathrm{CI})\end{array}$} & $8 \%$ & $69(49-88)$ & $160(95-223)$ & $165(123-210)$ & 0\%@120 min \\
\hline & $20 \%$ & $65(49-82)$ & $130(74-206)$ & $146(108-189)$ & $152(121-180)$ \\
\hline & $60 \%$ & $37(23-50)$ & $116(75-155)$ & $115(75-158)$ & $111(86-135)$ \\
\hline & $100 \%$ & $40(22-58)$ & $47(20-75)$ & $73(39-111)$ & $60(45-74)$ \\
\hline \multirow[t]{4}{*}{$\begin{array}{l}\text { LT50 } \\
(\text { min, 95\% CI) }\end{array}$} & $8 \%$ & $119(93-155)$ & $256(182-414)$ & $309(241-436)$ & $<70 \% @ 240$ min \\
\hline & $20 \%$ & $117(94-147)$ & $283(182-611)$ & $292(224-420)$ & $218(184-260)$ \\
\hline & $60 \%$ & $83(62-111)$ & $187(139-266)$ & $225(163-345)$ & $178(147-218)$ \\
\hline & $100 \%$ & $98(68-148)$ & $92(55-168)$ & $142(94-247)$ & $105(85-133)$ \\
\hline \multirow[t]{4}{*}{$\begin{array}{l}\mathrm{LT}_{75} \\
(\mathrm{~min}, 95 \% \mathrm{CI})\end{array}$} & $8 \%$ & $206(159-300)$ & $412(285-939)$ & $580(416-1027)$ & 100\%@480min \\
\hline & $20 \%$ & $209(164-289)$ & $616(347-2314)$ & $583(408-1066)$ & $314(263-403)$ \\
\hline & $60 \%$ & $189(164-289)$ & $304(221-525)$ & $440(297-901)$ & $285(231-382)$ \\
\hline & $100 \%$ & $241(158-502)$ & $185(112-518)$ & $278(176-712)$ & $187(147-261)$ \\
\hline
\end{tabular}

apearson's chi-square and the probability of $\mathrm{X}^{2}$. The probability of $>0.05$ indicates that the observed regression model is not significantly different from the expected model (i.e., a significant fit between the observed and expected regression models). $N=30$ for all concentrations except for $100 \%$ Nerol where $N=29$. 


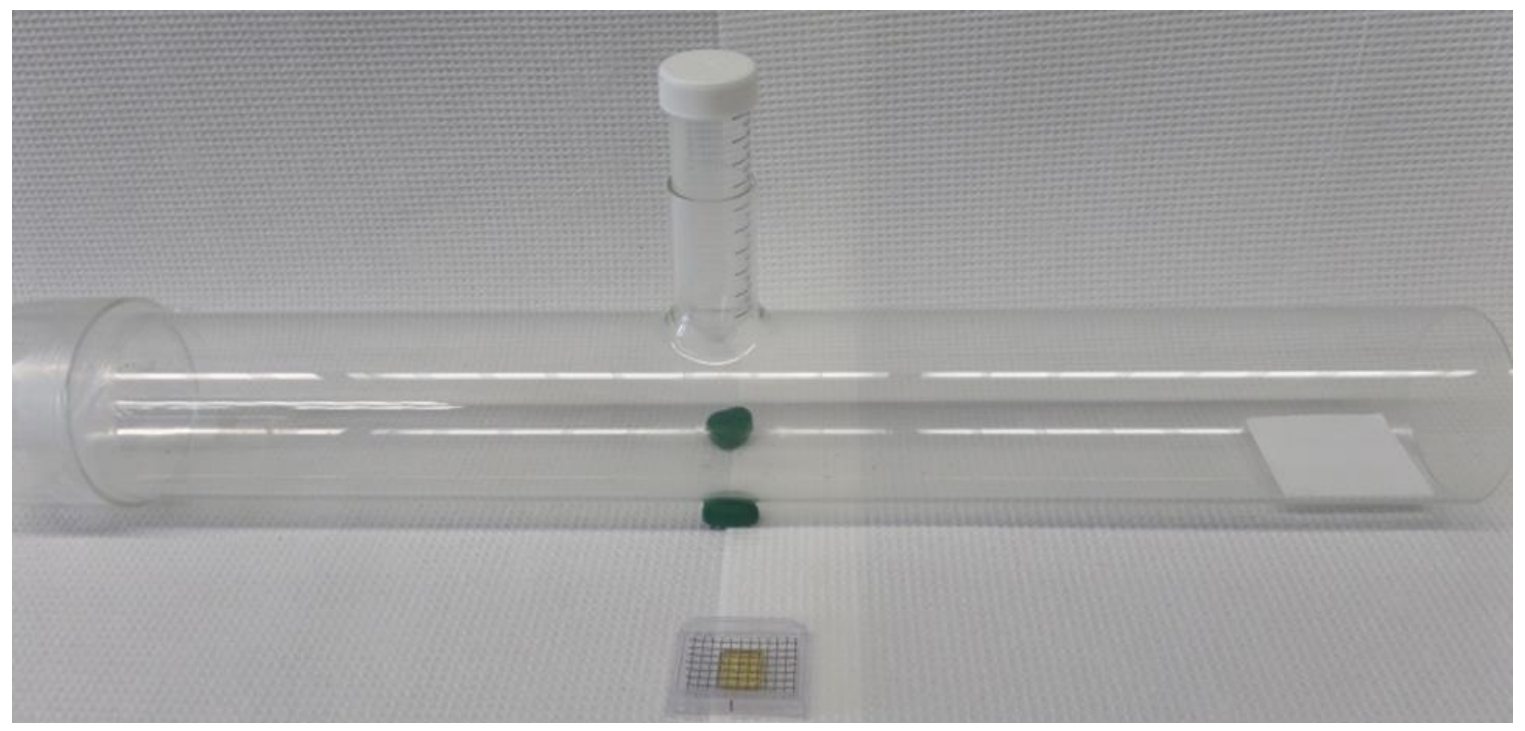

Figure 2. Toxicity and feeding inhibition glass tube arena with treated filter paper and feeding weigh boat. Arena is $60 \mathrm{~cm}$ long with a diameter of $10 \mathrm{~cm}$. Filter paper dimensions are $5 \times 6 \mathrm{~cm}$ and were treated with $400 \mu \mathrm{l}$ of acetone (control) or X\% treatment. The plastic weigh boat was filled with honey and a wire mesh was placed on top of the weigh boat. 

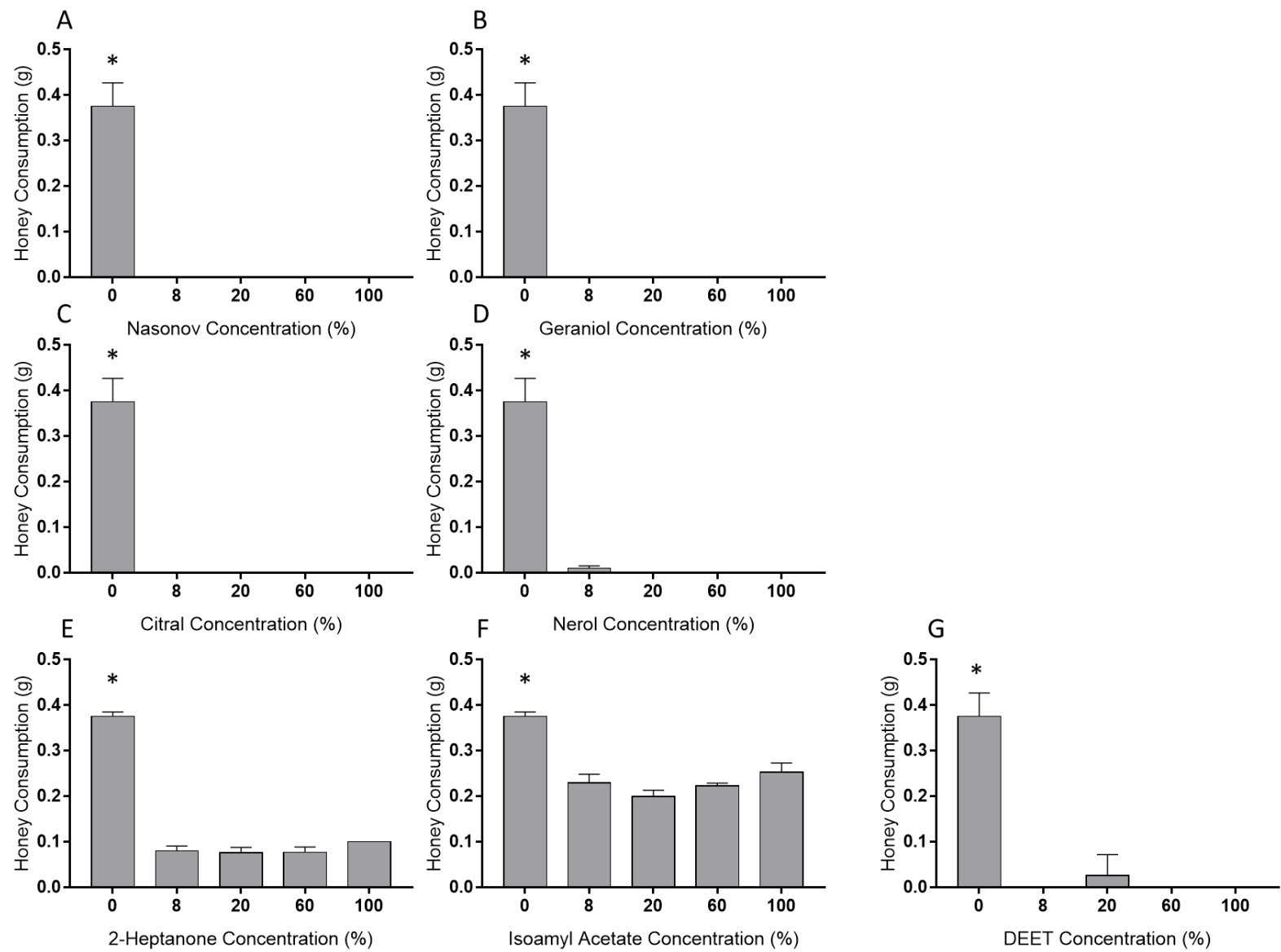

Figure 3. Effects of Nasonov pheromone, geraniol, citral, nerol, 2-heptanone, isoamyl acetate, and DEET on the honey consumption of honey bees. Honey bees were exposed to treated filter papers for $8 \mathrm{hrs}$ within a glass tube arena. Vertical bars indicate standard errors of the mean $(n=3)$. Significance was determined using an ANOVA $(P<0.0001)$ and indicated with asterisks. 


\subsection{REFERENCES}

Aizen, M. A., and L. D. Harder. 2009. The global stock of domesticated honey bees is growing slower than agricultural demand for pollination. Curr. Biol. 19: 915-918.

Atkins, W. M., R. L. MacDonald, T. P. McGovern, M. Beroza, and E. A. GreywoodHale. 1975. Repellent additives to reduce pesticide hazards to honeybees: laboratory Testing. J. Apic. Res. 14: 85-97.

Callbreath, J. 1900. The use of carbolic acid during spraying time. Glean. Bee Cult. 841.

Cresswell, J. E. 2011. A meta-analysis of experiments testing the effects of a neonicotinoid insecticide (imidacloprid) on honey bees. Ecotoxicology. 20: 149-157.

Dickens, J. C., and J. D. Bohbot. 2013. Mini review: Mode of action of mosquito repellents. Pestic. Biochem. Physiol. 106: 149-155.

Dimock, M. B., G. G. Kennedy, and W. G. Williams. 1982. F analogs of 2-tridecanone, a naturally occurring toxicant from a wild tomato. J. Chem. Ecol. 8: 837-842.

EPA. 2016. Guidance on exposure and effects: Testing for assessing risks to bees.

Fairbrother, A., J. Purdy, T. Anderson, and R. Fell. 2014. Risks of neonicotinoid insecticides to honeybees. Environ. Toxicol. Chem. 33: 719-731.

Free, J. B., J. A. Pickett, A. W. Ferguson, J. R. Simpkins, and M. C. Smith. 1985. Repelling foraging honeybees with alarm pheromones. J. Agric. Sci. 105: 255.

Gallai, N., J. M. Salles, J. Settele, and B. E. Vaissière. 2009. Economic valuation of the vulnerability of world agriculture confronted with pollinator decline. Ecol. Econ. 68: $810-821$.

Haarmann, T., M. Spivak, D. Weaver, B. Weaver, and T. Glenn. 2002. Effects of fluvalinate and coumaphos on queen honey bees (Hymenoptera: Apidae) in two 
commercial queen rearing operations. J. Econ. Entomol. 95: 28-35.

Inazuka, S. 1983. Monoterpenoids as repellents against the german cockroach (Blattella germanica L.). J. Pestic. Sci. 8: 293-299.

Johnson, R. M., M. D. Ellis, C. A. Mullin, and M. Frazier. 2010. Pesticides and honey bee toxicity - USA. Apidologie. 41: 312-331.

Katz, T. M., J. H. Miller, and A. A. Hebert. 2008. Insect repellents: historical perspectives and new developments. J. Am. Acad. Dermatol. 58: 865-871.

Keplinge, M., and W. Deichman. 1967. Acute toxicity of combinations of pesticides. Toxicol. Appl. Pharmacol. 10: 586-595.

Koul, O., S. Walia, and G. S. Dhaliwal. 2008. Essential oils as green pesticides: potential and constraints. Biopestic. Int. 4: 63-84.

Mullin, C. A., M. Frazier, J. L. Frazier, S. Ashcraft, R. Simonds, D. VanEngelsdorp, and J. S. Pettis. 2010. High Levels of miticides and agrochemicals in North American apiaries: implications for honey bee health. PLoS One. 5: e9754.

Playne, M. J., and B. R. Smith. 1983. Toxicity of organic extraction reagents to anaerobic bacteria. Biotechnol. Bioeng. 25: 1251-1265.

Potts, S. G., J. C. Biesmeijer, C. Kremen, P. Neumann, O. Schweiger, and W. E. Kunin. 2010. Global pollinator declines: trends, impacts and drivers. Trends Ecol. Evol. 25: 345-353.

Price, D. N., and M. S. Berry. 2008. Neurophysiological effects of naturally occurring defensive compounds on the freshwater snail Planorbis corneus: comparison with effects in insects. J. Chem. Ecol. 34: 994-1004.

Sanchez-Bayo, F., and K. Goka. 2014. Pesticide residues and bees - a risk assessment. 
PLoS One. 9: 1-16.

Schmidt, J. O. 1999. Attractant or pheromone: the case of Nasonov secretion and honeybee swarms. J. Chem. Ecol. 25: 2051-2056.

Seitz, N., K. S. Traynor, N. Steinhauer, K. Rennich, M. E. Wilson, J. D. Ellis, R. Rose, D. R. Tarpy, R. R. Sagili, D. M. Caron, K. S. Delaplane, J. Rangel, K. Lee, K. Baylis, J. T. Wilkes, J. A. Skinner, J. Pettis, and D. VanEngelsdorp. 2016. A national survey of managed honey bee 2014-2015 annual colony losses in the USA. J. Apic. Res. 54: 292-304.

Smith, K. M., E. H. Loh, M. K. Rostal, C. M. Zambrana-Torrelio, L. Mendiola, and P. Daszak. 2013. Pathogens, pests, and economics: drivers of honey bee colony declines and losses. Ecohealth. 10: 434-445.

Swale, D. R., B. Sun, F. Tong, and J. R. Bloomquist. 2014. Neurotoxicity and mode of action of N, N-diethyl-Meta-toluamide (DEET). PLoS One. 9: e103713.

Taylor, K. S., G. D. Waller, and L. A. Crowder. 1987. Impairment of a classical conditioned response of the honey bee (Apis mellifera L.) by sublethal doses of synthetic pyrethroid insecticides. Apidologie. 18: 243-252.

Teeters, B. S., R. M. Johnson, M. D. Ellis, and B. D. Siegfried. 2012. Using videotracking to assess sublethal effects of pesticides on honey bees (Apis mellifera L.). Environ. Toxicol. Chem. 31: 1349-1354.

Thompson, H. M. 2010. Risk assessment for honey bees and pesticides-recent developments and "new issues." Pest Manag. Sci. 66: 1157-1162.

Tison, L., M. L. Hahn, S. Holtz, A. Robner, U. Greggers, G. Bischoff, and R. Menzel. 2016. Honey bees' behavior is impaired by chronic exposure to the neonicotinoid 
thiacloprid in the field. Environ. Sci. Technol. 50: 7218-7227.

vanEngelsdorp, D., and M. D. Meixner. 2010. A historical review of managed honey bee populations in Europe and the United States and the factors that may affect them. J. Invertebr. Pathol. 103: S80-S95.

Williams, J. R. 2016. Biomarkers of oxidative stress in atrazine-treated honey bees: a laboratory and in-hive study. M.S. thesis. Virginia Polytechnic and State University, Virginia.

Williamson, S. M., and G. A. Wright. 2013. Exposure to multiple cholinergic pesticides impairs olfactory learning and memory in honeybees. J. Exp. Biol. 216: 1799-1807. 


\section{CHAPTER 3: VIDEO TRACKING PROTOCOL TO SCREEN DETERRENT CHEMISTRIES FOR HONEY BEES}

Larson, N. R., Anderson, T. D. Video Tracking Protocol to Screen Deterrent Chemistries for Honey Bees. J. Vis. Exp. (In Press), e55603, doi:10.3791/55603 (2017).

\subsection{ABSTRACT}

The European honey bee, Apis mellifera L., is an economically and agriculturally important pollinator that generates billions of dollars annually. Honey bee colony numbers have been declining in the United States and many European countries since 1947. A number of factors play a role in this decline including the unintentional exposure of honey bees to pesticides. The development of new methods and regulations are warranted to reduce pesticide exposures to these pollinators. One approach is the use of repellent chemistries that deter honey bees from a recently pesticide-treated crop. Here, we describe a protocol to discern the deterrence of honey bees exposed to select repellent chemistries. Honey bee foragers are collected and starved overnight in an incubator $15 \mathrm{~h}$ prior to testing. Individual honey bees are placed into Petri dishes that have either a sugar-agarose cube (control treatment) or sugar-agarose-compound cube (repellent treatment) placed into the middle of the dish. The Petri dish serves as the arena that is placed under a camera in a light box to record the honey bee locomotor activities using video tracking software. A total of 8 control and 8 repellent treatments were analyzed for a 10-min period with each treatment was duplicated with new honey bees. Here, we demonstrate that honey bees are deterred from the sugar-agarose cubes with a compound treatment whereas honey bees are attracted to the sugar-agarose cubes without an added compound.

\subsection{INTRODUCTION}


The European honey bee, Apis mellifera L., is an economic and agriculturally important insect that provides pollination services that are valued at more than $\$ 200$ billion globally (Gallai et al. 2009). In the United States and Europe, honey bee colony numbers have been declining. The United States has lost $c a .60 \%$ of managed honey bee colonies from 19472008 whereas Europe has lost ca. 27\% from 1961-2007 (Aizen and Harder 2009, vanEngelsdorp and Meixner 2010). There are a number of factors that might be responsible for the increased number of colony losses, including but not limited to, parasite infestations, pathogen infections, beekeeping practices, and pesticide use(vanEngelsdorp and Meixner 2010, Smith et al. 2013).

Honey bees may be exposed to pesticides via two main pathways. Pesticide exposure outside of the hive can occur when foraging individuals come into contact with crops that have been sprayed with chemicals for protection from pests. Pesticide exposure within the hive can occur when beekeepers utilize chemicals to control in-hive pests and pathogens, such as mites, bacteria, and microsporidia ${ }^{4}$. Pesticide residues have been identified within wax, pollen, and honey bee samples from 24 apiaries in the United States and Canada(Mullin et al. 2010, Li et al. 2015). Effects of pesticide contact to honey bees include acute toxicity as well as sublethal effects such as paralysis, disorientation, and behavioral and health changes (Gallai et al. 2009, Kakumanu et al. 2016). As modern agriculture requires the use of pesticides to maintain high crop yields, these chemicals will continue to be relied upon in the future(vanEngelsdorp and Meixner 2010). In order to better protect honey bees from pesticide exposures, there is a need for the development of new protocols and regulations(Mullin et al. 2010). One possible approach for protection 
is the use of repellents to reduce the exposure of honey bees to pesticides while foraging for food.

Insect repellents (IRs) have typically been used as personal bite protection measures against arthropod disease vectors (Katz et al. 2008). The most widely used and successful IR, developed more than 60 years ago, is DEET (Katz et al. 2008, Dickens and Bohbot 2013). It is considered to be the gold standard for insect repellent testing and is used by the World Health Organization and Environmental Protection Agency as a positive control for novel repellent screening (Lawrence et al. 2014). Additionally, DEET has been found to disperse honey bees from a threat to their colony (Collins et al. 1996). Current preferred attributes associated with personal IRs include: (1) lasting effect against a broad number of arthropods; (2) non-irritating to the user when applied to the skin or clothing; (3) odorless or pleasant odor; (4) no effect on clothing; (5) no oily appearance when applied to skin and to withstand sweating, washing, and wiping by the user; (6) no effect on commonly used plastics; and (7) chemically stable and affordable for widespread use (Brown and Hebert 1997). A repellent used for honey bees would only need a few of these attributes such as lasting effects, non-irritating to applicators, odorless or pleasant odor, chemically stable and affordable for widespread use, and non-toxic to honey bees. However, before exploring these attributes in depth, a method for screening compounds for repellency/deterrence in a high-throughput manner is needed. Here, we describe a protocol for a laboratory assay to screen compounds for the deterrence of honey bees, an important step in determining repellency. The following protocol is modified from a previous study describing a visual tracking method to assess the sublethal effects of 
pesticides on honey bees (Teeters et al. 2012). However, this protocol differs in that it is designed to measure the effects of candidate repellents that might deter honey bees from pesticide-treated crops. There are no recommended protocols for the laboratory testing of chemical deterrents for honey bees and, thus, this protocol provides a simple approach to screen such compounds.

\subsection{PROTOCOL}

1. Prepare Sugar-Agarose Cubes

1.1) Weigh out $8 \mathrm{~g}$ of sugar and place into a $50 \mathrm{~mL}$ Erlenmeyer flask.

1.2) Fill the Erlenmeyer flask with $20 \mathrm{~mL}$ of de-ionized water. Dissolve the sugar by swirling the flask.

1.3) Weigh $170 \mathrm{mg}$ of agarose and add it to the sugar solution.

1.4) Heat the sugar-agarose solution in a microwave on high for $25 \mathrm{~s}$. Dissolve the agarose into the sugar solution.

1.5) Allow the flask and sugar-agarose solution to cool.

Note: The flask should be cool to the touch, but do not allow the solution to solidify. 
1.5.1) To prepare a sugar-agarose cube for the control treatment, pour the semi-cooled sugar-agarose solution into a weigh boat mold.

Note: The weigh boat mold has the dimensions $1.5 \times 1.5 \times 0.3 \mathrm{~cm}$.

1.5.2) To prepare a sugar-agarose-compound cube for the repellent treatment, add the desired amount of compound to the semi-cooled solution (e.g., 1\% DEET in sugar-agarose solution $(\mathrm{v} / \mathrm{v}))$. Swirl the flask to mix in the compound and then pour the solution into a weigh boat mold.

Note: Eight control molds and eight test molds will be prepared at this point. Control molds do not contain repellent.

1.6) Cool the sugar-agarose cubes in the molds in a refrigerator for 5-10 min. Remove the solidified sugar-agarose cubes from the weigh boat molds and place them into a plastic container with a moistened paper towel.

1.7) Place the containers into a refrigerator for storage. The sugar-agarose cubes should be used within 7 days of preparation.

2. Programming the video tracking software and experimental setup 
2.1) Under Experiment Settings in the Experiment Explorer bar (in the tracking software, see table of materials), located on the left of the screen, make sure that the correct camera is selected and that the video recording is centered on the Petri dish arenas.

2.1.2) If the video recording needs to be centered, go into the settings of the camera and under the AOI controls and select the center $\mathrm{X}$ and center $\mathrm{Y}$ options.

2.2) Under Experiment Settings, change the number of arenas to 16. Under Tracked Features select Center-point detection.

2.3) Select Arena Settings to setup the arena desired for the assay.

2.3.1) Place the 16 Petri dishes in a $4 \times 4$ pattern on top of a light box, positioned under the camera (Figure 1).

Note: These dishes are used to create the recording arena and are empty.

2.4) In the Arena Settings, use the grab background button, located on the right-side tool panel, to take a picture of the 16 Petri dishes. This will be used as the template to setup the arena. 
2.5) Select "Create ellipse" from the tool bar at the top of the Arena Settings screen and create a circle that matches the diameter of one of the Petri dishes in the image grabbed. Place the Arena 1 marker into the circle (Figure 2).

2.6) Select "Zone Group 1" located under Arena 1 in the right-side tool panel. Select "Create Rectangle" from the tool bar at the top of the screen. Create a $30 \times 30$-pixel square using this and then position it at the center of the circle that was created in the previous step. Select "Add Zone" from the top tool bar and then click in the middle of the square. Move the Zone 1 marker so that it is in the square.

Note: The square created is the feeding zone and it is not a requirement that the feeding zone be exactly the same size as listed as long as it is slightly larger than the sugar-agarose cube.

2.7) Select "Arena 1" in the right-side tool panel and then click duplicate complete arena. From the drop-down menu select "All Other Arenas" and then click ok. Move the duplicated arena setups to the remaining Petri dishes in the grabbed image (Figure 3).

2.7.1) Select "Arena 1" and then select "Calibrate Scale" on the top tool bar. Draw the calibration line across the diameter of the Arena 1 Petri dish. Change the diameter measurement to $9 \mathrm{~cm}$ (diameter of the Petri dishes being used). Select "Validate Arena Settings" on the right-side tool panel. 
2.8) Select "Detection Settings" on the control bar on the left.

2.9) Select "Detection Settings 1" and then select "Grey Scaling" in the drop-down menu located on the right-side tool box. Under detection, set the range so that it is 0 to 83 (Figure $4 \mathrm{~A})$.

2.10) Right click "Detection Settings" and make a new setting named "Detection Settings 2". Make sure "Grey Scaling" is still selected, but do not change the other parameters.

Note: There will be large yellow circles in the video window over the arenas. This will aid in arranging the Petri dishes into the correct positions before recording (Figure 4B).

2.11) Select "Trial List" in the Experimental Explorer and click "Add Variable" in the top tool bar. Name the user defined variable as "Treatment". Select the "Predefined Values" drop down bar in the user-defined Treatment column and add C (control) and T (treatment) as Predefined Values. Select the "Add Trials" button located on the top tool bar. Add two trials, then for each arena select whether it is a control arena (C) or treatment arena (T) (Figure 5).

2.12) Select "Data Profile" under the Experiment Explorer panel. On the left-hand column select "Treatment" located under the "User-defined Independent Variables" heading. This will add a "Filter" box into the area with the flow chart. Select "C" in the pop up box and then place the newly created filter in between the "Start" box and "Result 1" box. The 
flowchart arrows should adjust so that they point from the start box to the filter and finally to the result 1 .

2.12.1) Repeat step 2.12, however this time select " $T$ " for the filter and then select "Result" under the "Common Elements" section. Move the newly created boxes into the flowchart area and connect the boxes with arrows (Figure 6).

2.13) Save the file.

3. Collect Honey Bee Individuals

3.1) Put on protective clothing and select a hive to collect honey bees (mostly foragers).

3.2) Remove the outer and inner cover of the hive. Use a hive tool to select a frame from the top hive body that does not contain brood. Gently lift the frame out of the hive box.

3.3) Inspect the frame for the honey bee queen. If she is not there, sweep the honey bee workers from the frame into a container for transport. Collect enough individuals to be able to run two complete trials for each compound to be tested (16 individuals are utilized per trial with controls).

Note: The collected individuals should primarily be foragers; however, there may be other aged individuals such as nurse bees included in the collection. 
3.4) Make a note of the time when the honey bees were removed. Transfer them into a $9 \times$ $7 \times 9 \mathrm{~cm}$ plastic box containing air holes and place them into an incubator at $32{ }^{\circ} \mathrm{C}$ and $70 \%$ relative humidity.

3.5) Starve the honey bees for $15 \mathrm{~h}$.

Note: This usually works best if the honey bees are collected the evening prior to being tested.

4. Conduct Visual Tracking Assay

4.1) Start up the visual tracking program and open the saved experimental file that was created for this assay.

4.2) Select "Detection Settings 2".

4.3) Place control sugar-agarose cubes into the centers of 8 of the 16 Petri dishes. Repeat this process with the sugar-agarose-compound (deterrent) cubes in the remaining 8 dishes.

4.4) Use forceps to remove a single honey bee from the plastic box and place it into one of the control Petri dishes. 
4.5) Repeat step 4.4 for the remaining 15 Petri dishes.

4.6) Place all of the dishes on to the light box and position them so that the arena detection areas (shown on the computer screen when detection setting 2 is selected) fit into each of the Petri dish arenas. Illuminate the light box from below by an array of LED lights set to the red spectrum. Surrounded the entire box and camera with a black plastic sheet to eliminate external light and reduce shadows within the arenas.

4.7) After the Petri dishes have been set, make sure "Detection Settings 1" is the selected setting.

Note: At this point the visual tracking software should be picking up only the honey bees within the Petri dish arenas.

4.8) Select "Acquisition" from the Experiment Explorer. Press the green "Start Trial" button located in the Acquisition Control pop up box on the right to begin recording.

4.9) Run the assay for 10 min and then click the red "Stop Trial" button on the Acquisition Control pop up box to stop recording.

4.10) Remove the individuals from the Petri dishes and place them into a separate container so that they are not tested twice. 
4.11) Repeat steps 4.4-4.10 for the second trial on the same compound.

Note: This method can be modified to increase the number of honey bees per treatment as needed by the user.

4.14) Select Statistics in the Experiment Explorer and then click calculate.

4.15) Export the data to a data manager software and then analyze the data for significance using a using a one-way analysis of variance with a Tukey's post-test, and an unpaired ttest using preferred statistical software program.

\subsection{RESULTS}

A video tracking protocol was developed to record the amount of time the honey bees spent in a target zone with either sugar-agarose (control treatment) or sugar-agarose-compound cube (deterrent treatment). The recorded time was analyzed using a statistical software program and the mean time spent \pm standard error in the target zone is reported as a bar graph. DEET, the gold standard for insect repellent/deterrent testing, was used in this protocol as a positive control. The honey bees provided with a sugar-agarose cube (negative control) spent $343 \pm 26 \mathrm{~s}$ in the target zone whereas the honey bees provided with a sugar-agarose-DEET (repellent) spent $16 \pm 4 \mathrm{~s}$ in the target zone (Fig. 7). DEET significantly reduced the amount of time spent by the honey bees in the target zone by $c a$. $95 \%$ compared to that of the control treatment. 
Compounds that were of interest to determine deterrence to honey bees were then screened through this protocol. Figure 8A represents a compound that does not deter honey bees from the food source in the target zone. The mean time spent by honey bees in the target zone in control dishes was $c a .352 \pm 60 \mathrm{~s}$, compared to $c a .282 \pm 43 \mathrm{~s}$ for honey bees within Petri dishes that had sugar-agarose cubes infused with compound A. Figure 8B represents a compound other than DEET having similar deterrence effects on the individual honey bees. Honey bees within in the control Petri dishes remained in the target zone for a mean time of $c a .493 \pm 31$ s, compared to $c a .23 \pm 3$ s for honey bees within Petri dishes containing a sugar-agarose cube infused with compound B. These results validate the use of this protocol for screening of chemical deterrents for honey bees. Prior to running this protocol with compounds of interest, it may be necessary to conduct a time-course trial to determine the amount of starvation time for the honey bees.

\subsection{DISCUSSION}

This visual tracking protocol provides a simple approach to screen chemical deterrents for honey bees in a relatively quick and easy manner. There are no recommended protocols for the laboratory testing of chemical deterrents for honey bees. Previous semi- and fullfield studies have examined honey bee repellents (Free et al. 1985, Vallet et al. 1991); however, the described protocols are time consuming, labor intensive, and require additional facility resources outside of a general laboratory. This protocol was designed as a pre-requisite evaluation of chemical deterrents prior to semi- or full-field testing of such compounds with honey bees. 
There are challenges when evaluating chemical deterrents for honey bees outside of the hive. For example, honey bees are social insects that that rely on pheromones within the hive that affect behavior (Vallet et al. 1991). This protocol requires the use of individuals that no longer receive pheromone cues, in addition to starvation. Starvation is required to standardize the feeding responses of individual honey bees. The starvation time was determined by a $24-\mathrm{h}$ time course study. It should be noted that starvation can have detrimental effects on the individual honey bees. For example, the honey bees become lethargic $18 \mathrm{~h}$ after collection from the hive. Based on these observations, the honey bees were starved for $15 \mathrm{~h}$ after collection from the hive.

The critical steps involved with this protocol to avoid unsuccessful screening include: (1) conducting the tests after at least $12 \mathrm{~h}$ of starvation; (2) avoid conducting tests after starvation exceeds 18-19 h, as this decreases honey bee vigor; (3) replace the control individuals for each trial; and (4) manage of external light and control shadows within the arenas. Additionally, the Petri dishes should be replaced before screening a new compound screen. Occasionally, a honey bee will defecate within the Petri dish during recording. This usually does not interfere with the recording or data collection. All Petri dishes should be washed thoroughly after each screen to remove sugar-agarose and compound residues as well as honey bee feces.

This protocol is primarily designed to screen compounds for deterrence to honey bees, but can be easily adapted to discern deterrence in other insect species. A major benefit of using the visual tracking software is that it makes a full video recording for the screening of each 
compound. If there is a need to review and analyze each recording, the investigator can select the file of interest and quickly conduct the screen again with the same or new parameters. The visual tracking software also has the capability to detect individual insects smaller than a honey bee. However, this may require a reduced field of vision for the camera field and fewer arenas to be recorded in a single screen. The strength of this protocol is the ability to screen compounds within minutes for deterrent effects in a laboratory setting. As such, time and money could be saved by reducing a compound library of interest to a select number of candidates for field testing. 


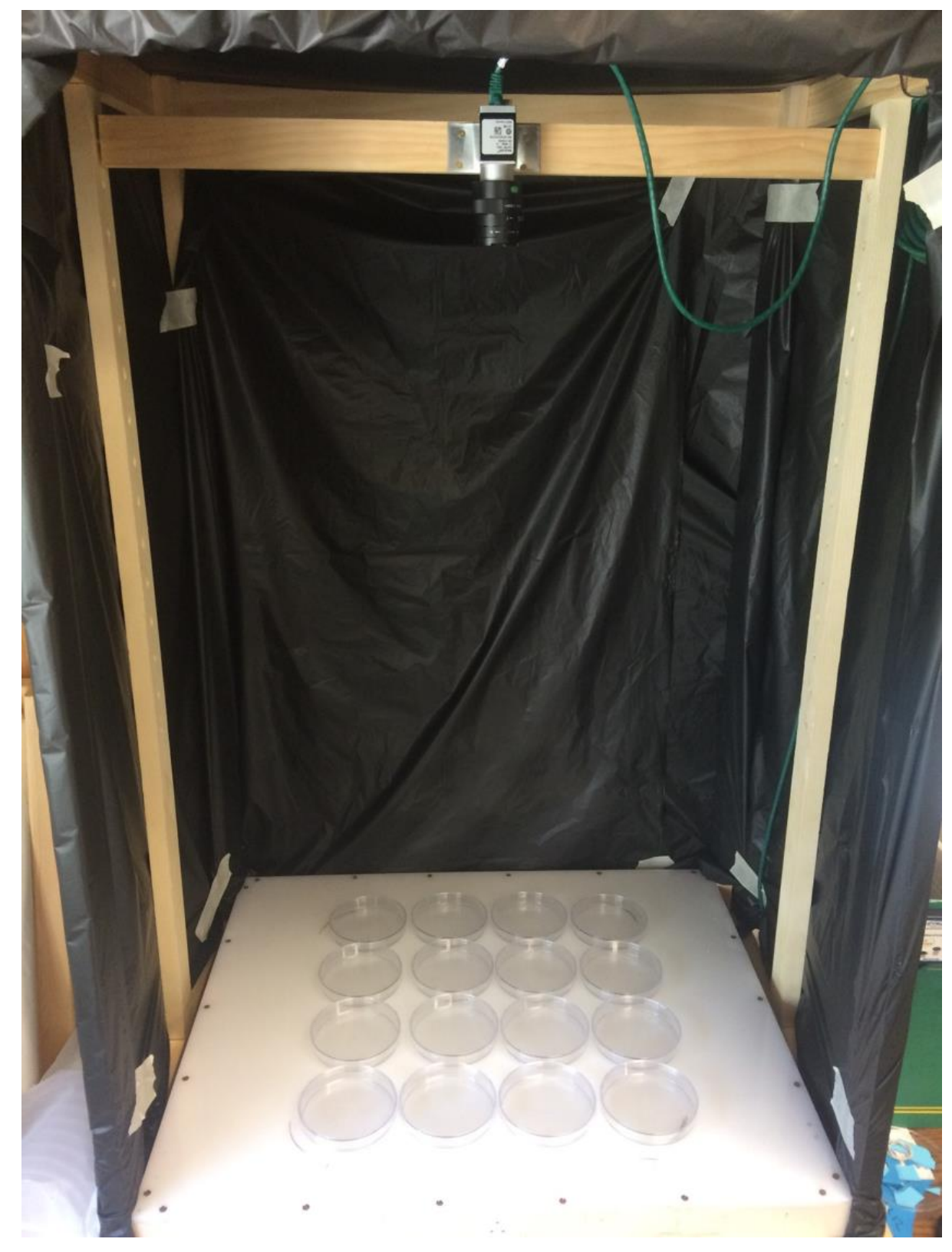

Figure 1: Petri dish arrangement on the light box. Petri dishes are arranged in a $4 \times 4$ block on top of the light box. This arrangement provides easy identification of the control and repellent treatments for the visual tracking protocol. 


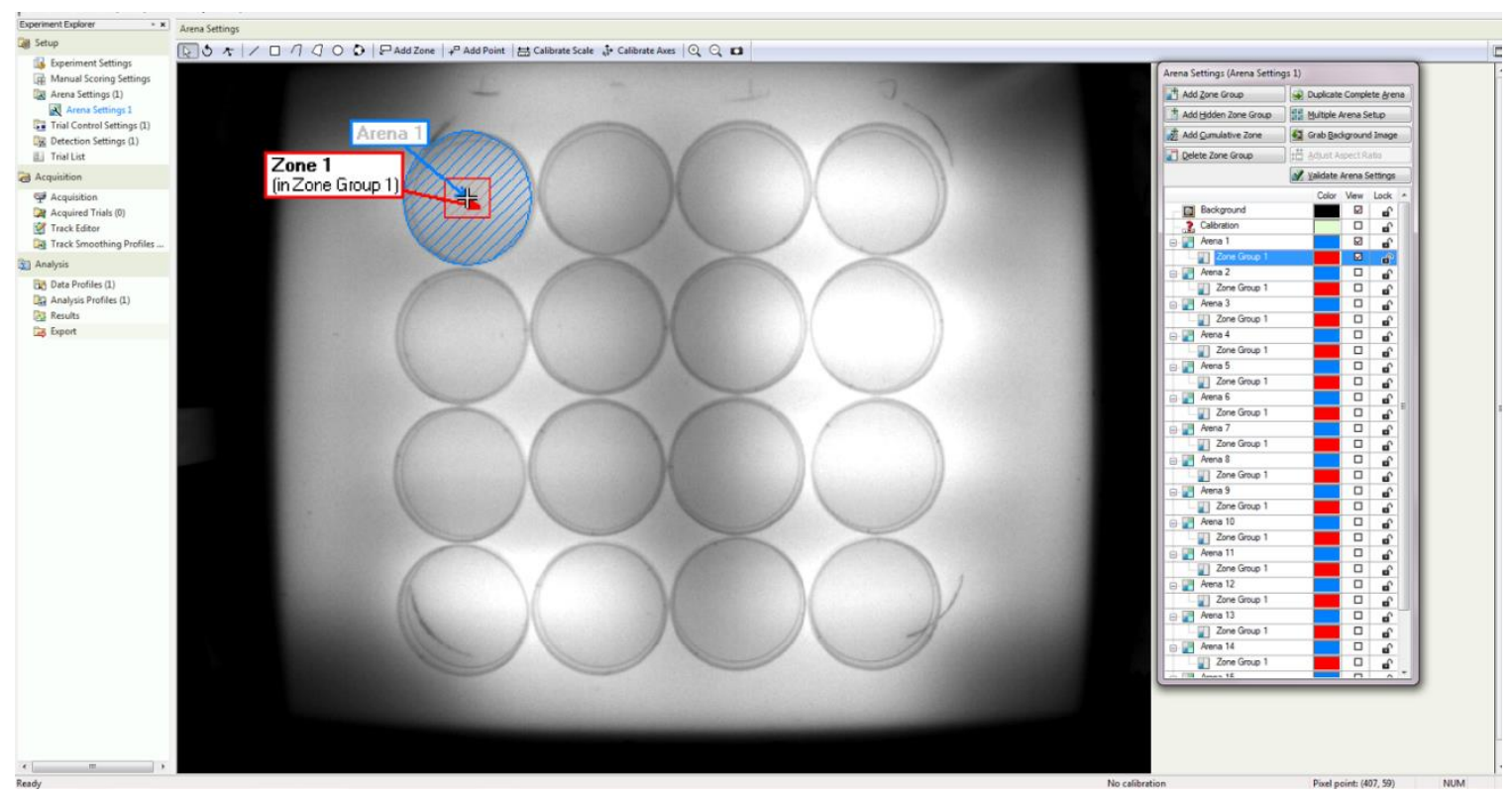

Figure 2: Screenshot of the Visual Tracking Software Arena Settings. The observation of diagonal stripes within the circle provides confirmation of the detection area in the circle. A Zone 1 marker is provided for each square and defines the target zone for each Petri dish. 


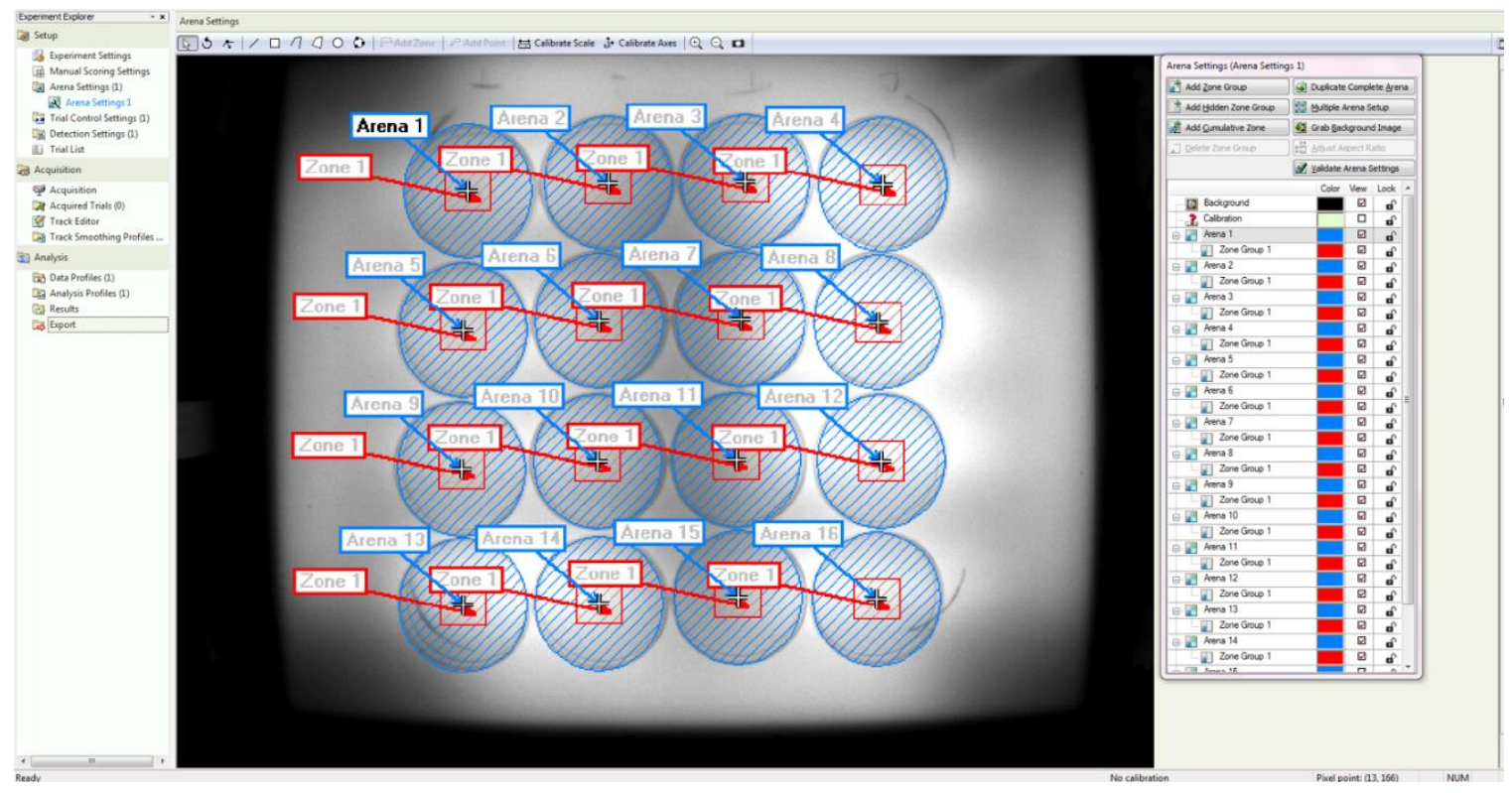

Figure 3: Screenshot of Completed Arena Settings. The completed arena settings should look like this example. 


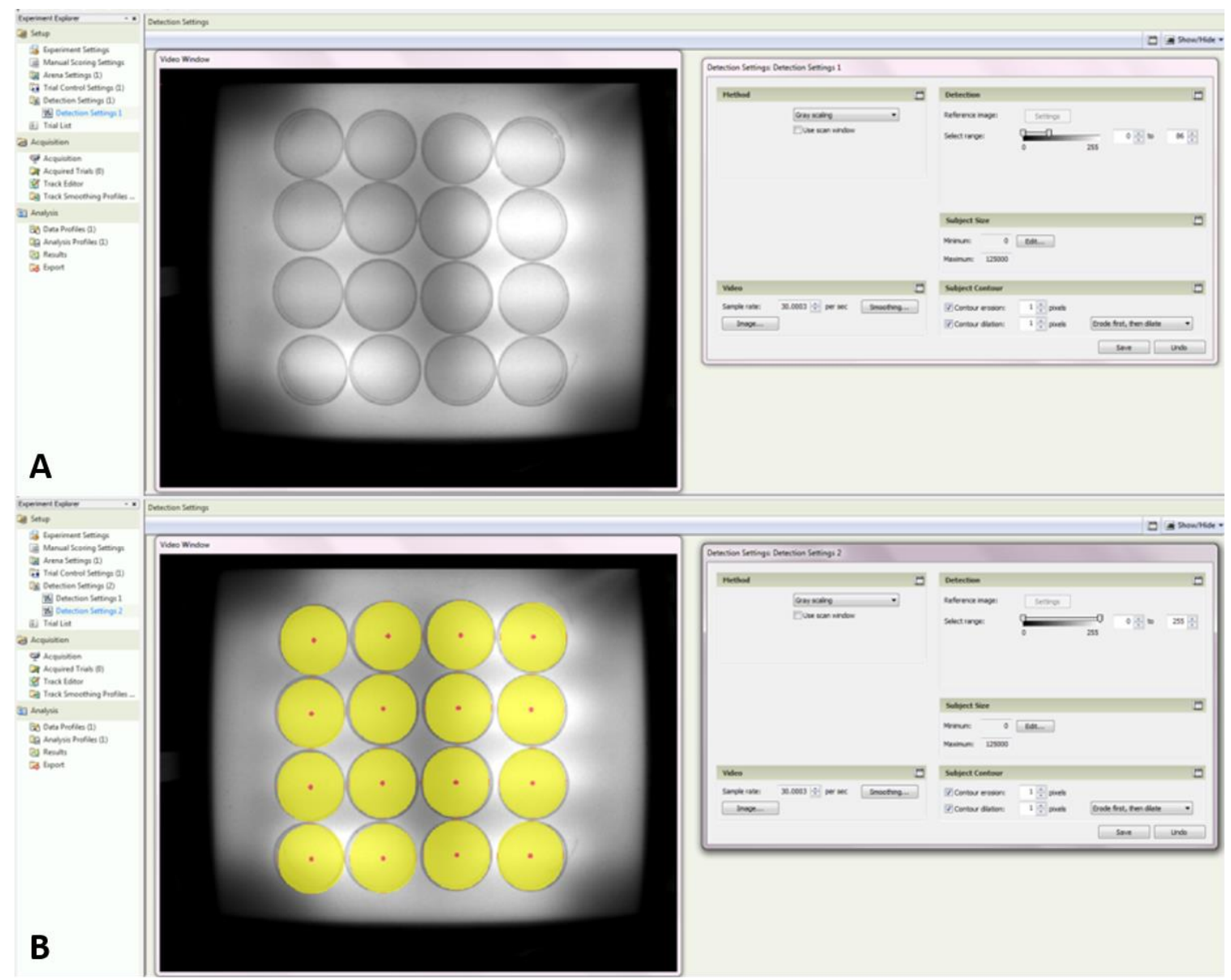

Figure 4: Screenshot of the Detection Settings 1 and 2. (A) Shows what the arena will look like with the grey scaling corrected for a honey bee subject in a petri dish. (B) Shows the arena detection area so that positioning of the petri dishes can be done between trials. 


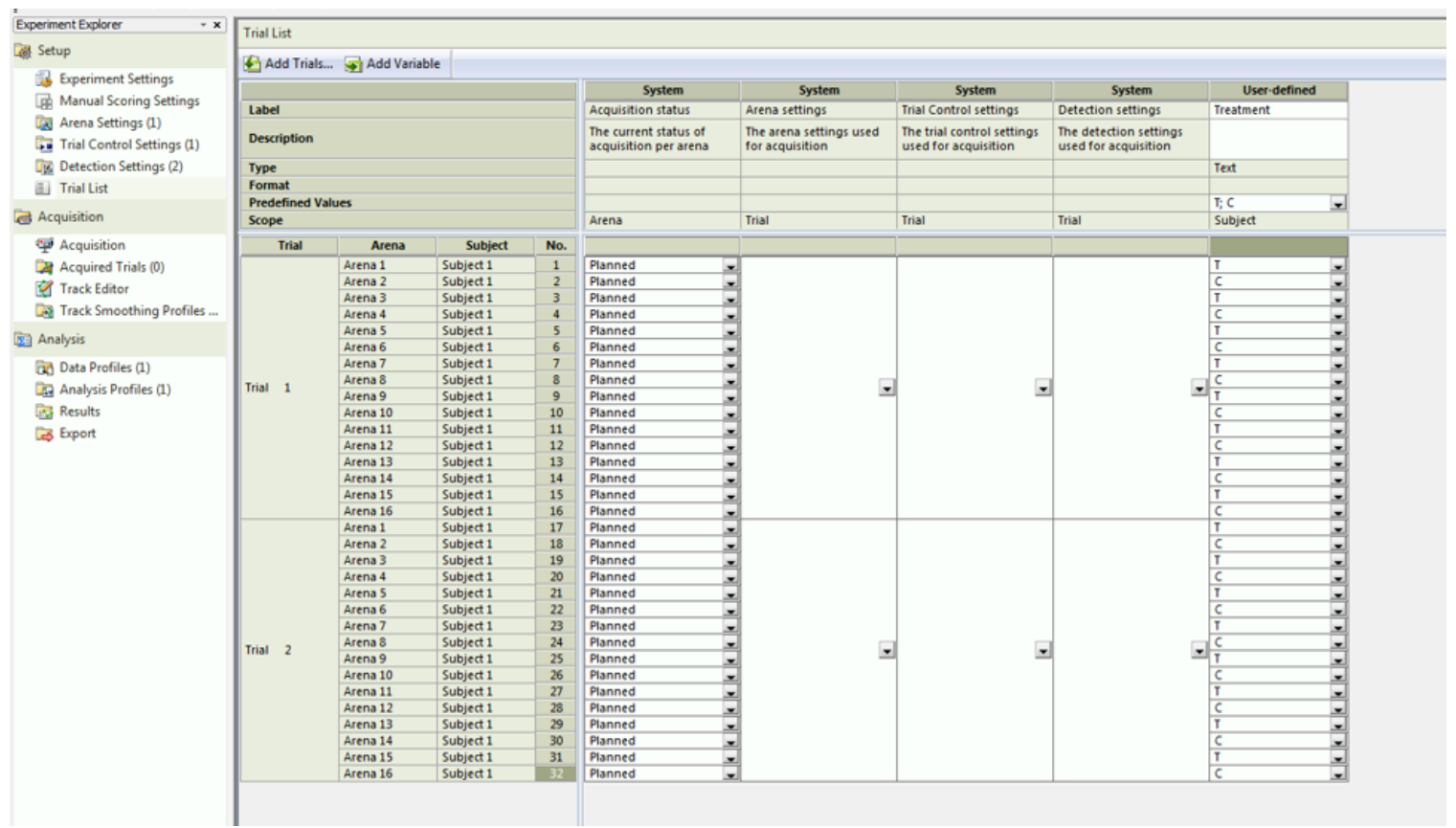

Figure 5: Screenshot of the Trial List. Labeling the arenas correctly is important here as the program uses the information here to separate the data to statistically analyze it.

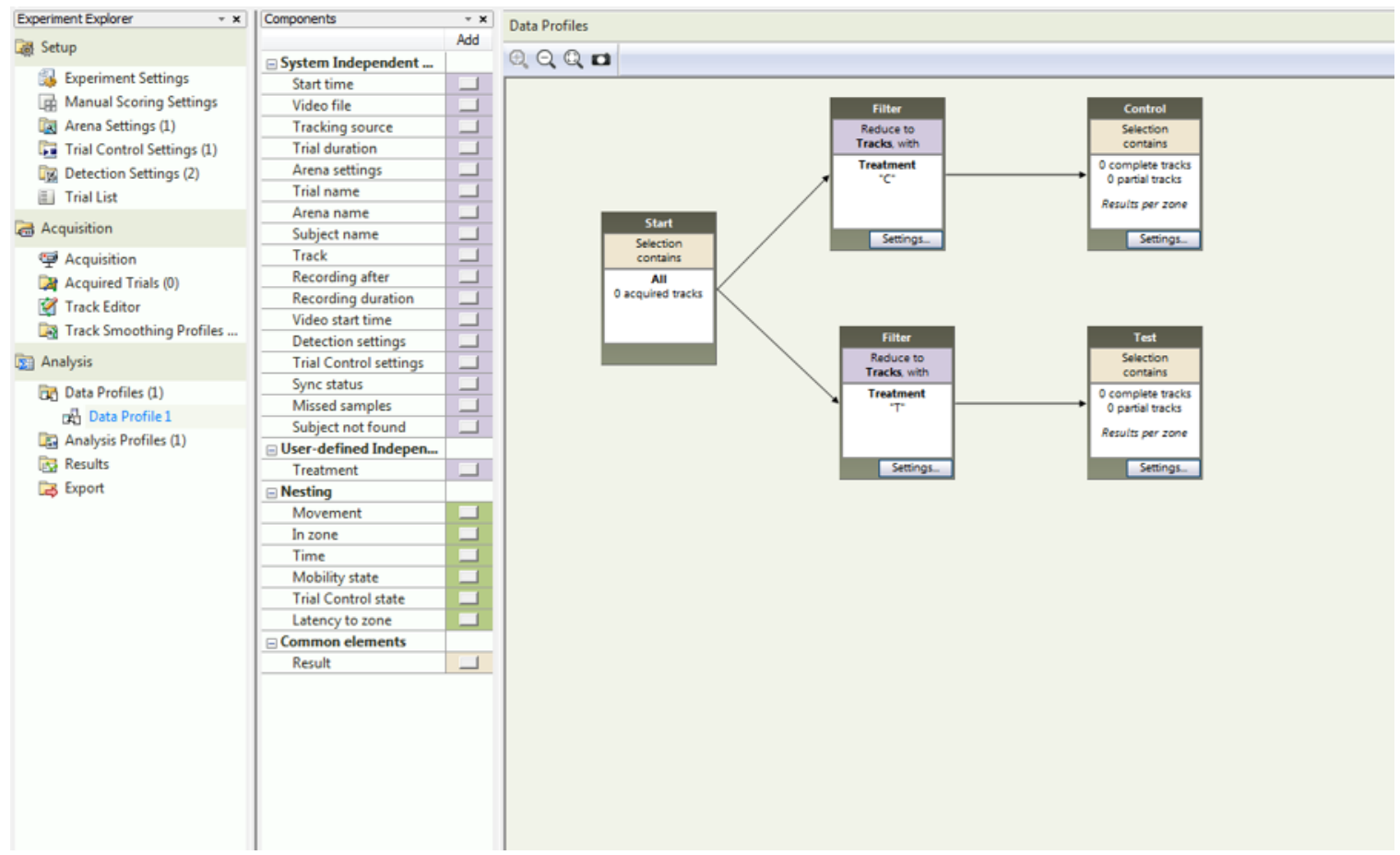

Figure 6: Screenshot of Data Profile. This shows how the flowchart should be set up to get the appropriate separation in the statistical analysis. 


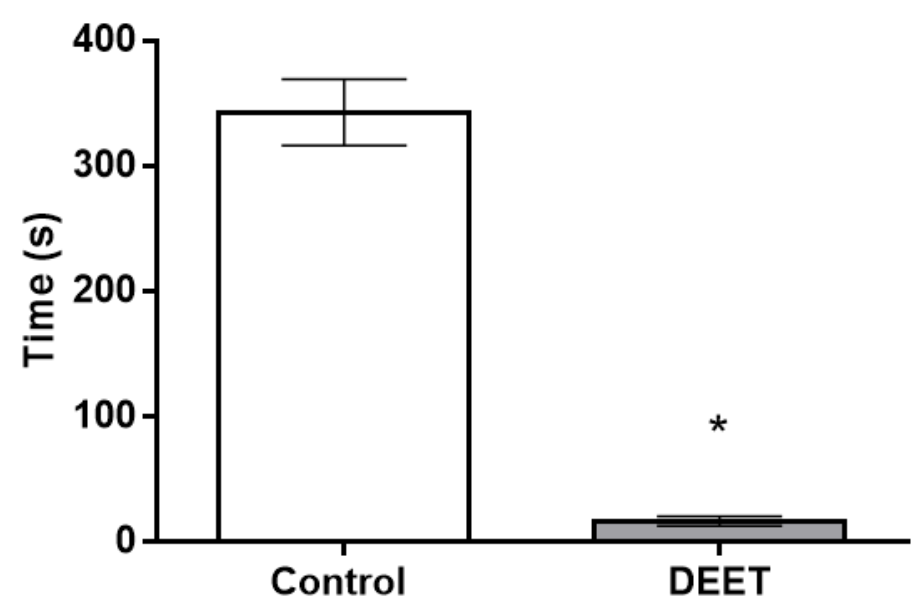

Figure 7: Example Results from the Visual Tracking Software Deterrence Protocol with Positive Control DEET. Honey bees are collected from a hive in the evening and removal time is recorded. They are then transferred into a plastic container containing air holes. The box is placed into an incubator set at $32{ }^{\circ} \mathrm{C}$ and held overnight for $15 \mathrm{~h}$. The following morning, individuals are placed into petri dishes containing either a control sugar-agarose cube or a DEETinfused sugar-agarose cube. The deterrence protocol described is then run. The results shown in this figure are typical for the repellency gold standard-DEET. From this figure, we see that the average amount of time a starved honey bee will spend in the feeding zone with a control cube (ca. $343 \mathrm{~s})$ is significantly greater $(\mathrm{P}<0.0001)$ than the average time an individual spends in the feeding zone with a cube impregnated with DEET ( $c a .16 \mathrm{~s}$ ). 

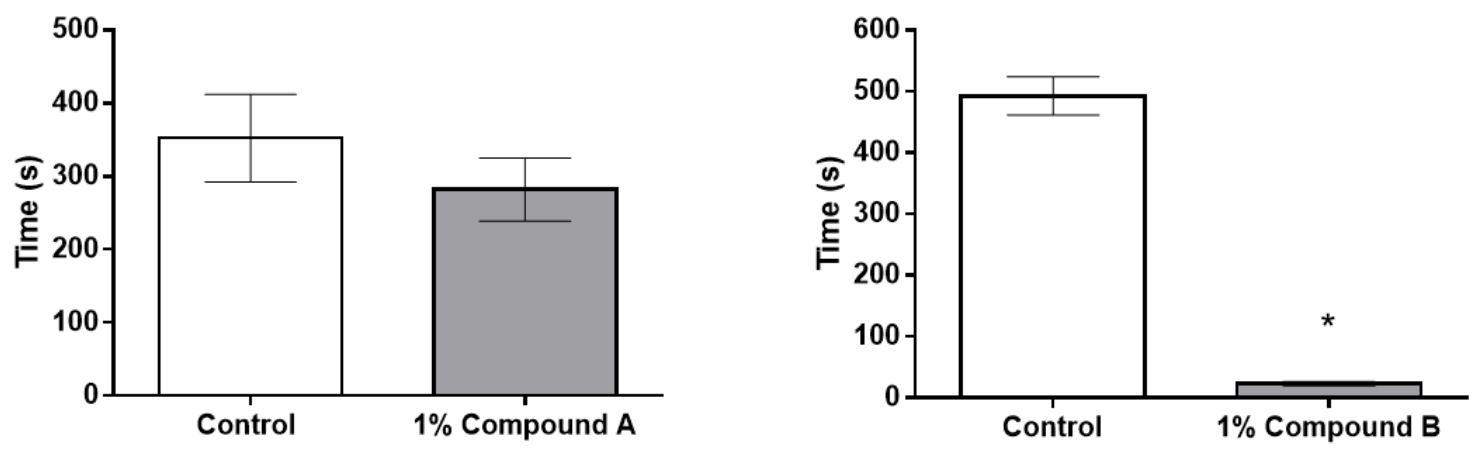

Figure 8: Example Results from Visual Tracking Software Tested Compounds. (A) Represents data that shows the mean time spent by individuals in the target zone in control dishes ( $c a .352 \mathrm{~s}$ ) is not significantly difference from the mean time spent in the target zone in tested dishes with compound A ( $c a .282 \mathrm{~s}$ ). (B) Represents data showing significant differences in mean time spent in the target zone between control cubes ( $c a .493 \mathrm{~s}$ ) and compound B-infused cubes (ca. $23 \mathrm{~s}$ ). An unpaired t-test was run to determine significance (P < 0.0001; DF 15). 


\section{6 REFERENCES}

Aizen, M. A., and L. D. Harder. 2009. The global stock of domesticated honey bees is growing slower than agricultural demand for pollination. Curr. Biol. 19: 915-918.

Brown, M., and A. A. Hebert. 1997. Insect repellents: An overview. J. Am. Acad. Dermatol. 36: 243-249.

Collins, A. M., W. L. Rubink, J. I. Cuadriello Aguilar, and R. L. Hellmich II. 1996. Use of insect repellents for dispersing defending honey bees (Hymenoptera: Apidae). J. Econ. Entomol. 89: 608-613.

Dickens, J. C., and J. D. Bohbot. 2013. Mini review: mode of action of mosquito repellents. Pestic. Biochem. Physiol. 106: 149-155.

Free, J. B., J. A. Pickett, A. W. Ferguson, J. R. Simpkins, and M. C. Smith. 1985. Repelling foraging honeybees with alarm pheromones. J. Agric. Sci. 105: 255.

Gallai, N., J. M. Salles, J. Settele, and B. E. Vaissière. 2009. Economic valuation of the vulnerability of world agriculture confronted with pollinator decline. Ecol. Econ. 68: $810-821$.

Kakumanu, M. L., A. M. Reeves, T. D. Anderson, R. R. Rodrigues, M. A. Williams, and M. A. Williams. 2016. Honey bee gut microbiome is sltered by in-hive pesticide exposures. Front. Microbiol. 7: 1-11.

Katz, T. M., J. H. Miller, and A. A. Hebert. 2008. Insect repellents: historical perspectives and new developments. J. Am. Acad. Dermatol. 58: 865-871.

Lawrence, K. L., N. L. Achee, U. R. Bernier, K. D. Mundal, and J. P. Benante. 2014. Field evaluations of topical arthropod repellents in North, Central, and South America. J. Med. Entomol. 51: 980-988. 
Li, Y., R. A. Kelley, T. D. Anderson, and M. J. Lydy. 2015. Talanta development and comparison of two multi-residue methods for the analysis of select pesticides in honey bees , pollen , and wax by gas chromatography - quadrupole mass spectrometry. Talanta. 140: 81-87.

Mullin, C. A., M. Frazier, J. L. Frazier, S. Ashcraft, R. Simonds, D. VanEngelsdorp, and J. S. Pettis. 2010. High levels of miticides and agrochemicals in North American apiaries: Implications for honey bee health. PLoS One. 5: e9754.

Smith, K. M., E. H. Loh, M. K. Rostal, C. M. Zambrana-Torrelio, L. Mendiola, and P. Daszak. 2013. Pathogens, pests, and economics: Drivers of honey bee colony declines and losses. Ecohealth. 10: 434-445.

Teeters, B. S., R. M. Johnson, M. D. Ellis, and B. D. Siegfried. 2012. Using videotracking to assess sublethal effects of pesticides on honey bees (Apis mellifera L.). Environ. Toxicol. Chem. 31: 1349-1354.

Vallet, A., P. Cassier, and Y. Lensky. 1991. Ontogeny of the fine structure of the honeybee (Apis mellifera L.) workers and the pheromonal activity of 2-heptanone. J. Insect Physiol. 37: 789-804.

vanEngelsdorp, D., and M. D. Meixner. 2010. A historical review of managed honey bee populations in Europe and the United States and the factors that may affect them. J. Invertebr. Pathol. 103: S80-S95. 


\section{CHAPTER 4: SPATIAL EFFECTS OF NASONOV PHEROMONE AND ITS CONSTITUENTS ON HONEY BEES}

\subsection{ABSTRACT}

There are several stressors that reduce the health of honey bee colonies and increase colony losses. This results in increased expenditures by beekeepers to recoup the losses that they face to maintain successful beekeeping operations. Pesticide exposures have been found to have several different effects on bees including reduced immunocompetence, negative effects on navigation, and reduced social communication. Pollination protection focuses on pesticide application guidelines. However, there is a need for a more active approach to protect foraging bees from pesticide applications. One approach is the use of a repellent in combination with a pesticide application. This study has found that honey bee pheromonal compounds elicit repellent like effects within a laboratory video-tracking assay. Individual bees were placed into Petri dishes that contained a sugar-agarose food cube. Control treatments were made just as sugar-agarose cubes and test cubes were treated with test compound. A $1 \%(\mathrm{v} / \mathrm{v})$ concentration of synthetic Nasonov pheromone, was shown to reduce the time an individual bee would spend within a feeding zone by $94 \%$ compared to untreated controls. The constituents that make up Nasonov pheromone: citral, nerol, and geraniol also reduced time spent within a feeding zone by up to $97 \%$ compared to controls. The alarm pheromone isoamyl acetate only reduced feeding by $48 \%$ compared to controls. Comparatively, the repellent standard DEET reduced feeding by $97 \%$.

\subsection{INTRODUCTION}

Managed honey bee colony operations within the United States and Europe have suffered significant losses since the 1940s. (Aizen and Harder 2009, vanEngelsdorp and Meixner 
2010). Beekeepers are attempting to manage these losses by splitting surviving colonies as well as purchasing new colonies each year, increasing their operational costs (Seitz et al. 2016).(Seitz et al. 2016, USDA-NASS 2017). This has slowed down the regional decline in the US; however, US beekeepers still lose approximately 50\% of their colonies each year, which exceeds the $19 \%$ that surveyed beekeepers deemed as "acceptable" in 2015 (Seitz et al. 2016, USDA-NASS 2017). . There are many factors associated with bee colony loss in the US. The most common factors reported by beekeepers are queen failure, starvation, varroa mite infestation, and weak overwintering colonies (Seitz et al. 2016). Although, there are other stressors that should be taken into consideration, including beekeeping practices, genetic variability of colonies, weak queens, weather and climate, adequate bee forage, and pesticides (vanEngelsdorp and Meixner 2010).

Bees encounter pesticides by several means. The two most direct pathways are from the grower-applied pesticides to crops visited by foraging bees and from beekeeper-applied pesticides in the hive for managing pests and pathogens (Johnson et al. 2010, vanEngelsdorp and Meixner 2010, Smith et al. 2013). When foraging, adult bees can pick up pesticides from various sources, including nectar, pollen, guttation fluid, soil, residues on leaves from foliar sprays, and dusts from treated seeds. Foraging bees can transport pesticide residues back to the hive where the remainder of the colony can be exposed to the pesticides via processed nectar and pollen (Thompson 2010, Cresswell 2011, Fairbrother et al. 2014). A recent risk assessment by Sanchez-Bayo and Goka (2014) identified 161 pesticide residues in bee hives across the world. This study reported 52\% 
of the detected residues to be insecticides whereas $25 \%, 17 \%$, and $6 \%$ of the residues detected in the hives were fungicides, herbicides, and acaricides, respectively.

Bee exposure to pesticides can have a variety of detrimental outcomes, including acute and sublethal effects (vanEngelsdorp and Meixner 2010). The two most common pesticides found in hives are coumaphos and tau-fluvalinate, the miticides used in Checkmite+ ${ }^{\circledR}$ and $\operatorname{Apistan}^{\circledR}$, respectively. These acaricides have been shown to affect olfactory learning, downregulate immune genes, and decrease locomotor activity in bees (Taylor et al. 1987, Mullin et al. 2010, Teeters et al. 2012, Williamson and Wright 2013, Sanchez-Bayo and Goka 2014, Cizelj et al. 2016, Garcia et al. 2017). A recent study found that chronic exposures to the neonicotinoid thiacloprid affected bee navigation, motivation to forage, and social communication (Tison et al. 2016). Neonicotinoid exposure has also been shown to decrease the immune system of bees and, in turn, increase their susceptibility to disease (Brandt et al. 2016, Christen et al. 2016). With the continued use of pesticides in agriculture, it is important that new regulations and practices be developed to ensure the protection of these important pollinators (Mullin et al. 2010, vanEngelsdorp and Meixner 2010). The development of a bee deterrent or repellent could provide one such method for protecting these pollinators from pesticide exposures (Atkins et al. 1975, Free et al. 1985).

Typically, insect repellents have been developed for personal bite protection against disease-transmitting arthropods, such as mosquitoes and ticks (Katz et al. 2008). However, there are studies that demonstrate natural compounds to repel bees (Free et al. 1985, Vallet 
et al. 1991). Interestingly, these compounds are alarm pheromones produced by bees (Boch et al. 1962, Shearer and Boch 1965). Nasonov pheromone consists of citral, geraniol and nerol that is produced by bees (Boch and Shearer 1964, Shearer and Boch 1966, Schmidt 1999). Nasonov pheromone has been found to elicit behavioral effects on other organisms, such as parasitic mites, freshwater snails, and stingless bees (Johnson and Wiemer 1982, Hoppe and Ritter 1988, Price and Berry 2008). Based on these findings, it was hypothesized that these pheromonal compounds will repel bees from treated food sources. This study provides a video-tracking experiment for bees exposed to sugar-agar cubes treated with Nasonov pheromone, citral, geraniol, nerol, and DEET. The data presented here provides evidence that these compounds significantly reduce the amount of time the bees visit the treated sugar-agar cubes compared to the untreated cubes.

\subsection{MATERIALS AND METHODS}

\subsubsection{Honey bees}

Bees were maintained in an apiary at Prices Fork Research Facility of Virginia Tech (Blacksburg, VA) using standard beekeeping practices. Worker bees were collected from frames located in the uppermost super of a colony to ensure that a large number of newly emerged and nurse bees were not collected prior to testing the repellent compounds. The collected worker bees were maintained in an incubator at $32{ }^{\circ} \mathrm{C}$ with $c a .70 \%$ relative humidity and starved overnight prior to the compound exposures and video-tracking recordings.

\subsubsection{Bee pheromones and DEET}


Synthetic Nasonov Pheromone was purchased from Dadant \& Sons Beekeeping Equipment (Chatham, VA). Geraniol, citral, nerol, isoamyl acetate, and agarose were purchased from Sigma-Aldrich Chemical Co. (St. Louis, MO). OFF! Deep Woods® Sportsmen was used at the source for DEET (98\% active ingredient; $N, N$-Diethyl-metatoluamide).

\subsubsection{Video-tracking software and recordings}

A Basler acA-1300-60gm camera and EthoVision ${ }^{\circledR}$ XT video recording software was used for the recordings (Noldus, Leesburg, VA). A light box was used to illuminate the assay arena with Cryon LED lights (Model HTP904E, Chatsworth, CA) set to the red spectrum to avoid bee light bias. The light box was and camera system was covered with a black plastic sheet to eliminate ambient light.

A video-tracking protocol described by Larson and Anderson (2017) was used to evaluate the effects of heterocyclic amines (HCAs) and DEET on the feeding behavior of bees. Briefly, sugar-agarose cubes were prepared by dissolving $8 \mathrm{~g}$ of sugar into $20 \mathrm{ml}$ deionized $\mathrm{H}_{2} \mathrm{O}$ followed by the addition of $170 \mathrm{mg}$ agarose. The sugar-agarose mixture was heated and the solution was poured into $1.5 \times 1.5 \times 0.3 \mathrm{~cm}$ molds. The pheromones and DEET were added to the sugar-agarose solution, at the desired concentrations, prior to pouring the solution into the molds. A total of 8 control sugar-agarose cubes (no pheromones or DEET) and 8 compound pheromones or DEET sugar-agarose cubes were placed into the centers of $9 \mathrm{~cm}$ diameter Petri dishes (i.e., feeding zone). The Petri dishes were positioned on top of the light box in a $4 \times 4$ pattern and individual bees were placed into each of the 
Petri dishes. The EthoVision ${ }^{\circledR}$ video-tracking recordings were conducted with bees exposed to the control and compound sugar-agarose cubes for $10 \mathrm{~min}$ followed by another set of recordings with new bees exposed to the same cubes for another 10 min. Each videotracking recording was conducted in duplicate. The time spent by the bees in the feeding zones was calculated by the EthoVision ${ }^{\circledR}$ video-tracking software and the data were statistically analyzed using a one-way ANOVA with a Tukey's post hoc test to determine significantly different means in GraphPad Prism (La Jolla, CA) software.

\subsection{RESULTS}

The amount of time spent by bees in the feeding zones containing sugar-agarose cubes treated with pheromonal compounds and DEET at $0.1 \%(\mathrm{v} / \mathrm{v})$ was observed as isoamyl acetate $($ more time spent $)<$ nasonov pheromone $=$ DEET $=$ citral $<$ geraniol $<$ nerol $($ less time spent) (Fig. 1A). Bees were recorded in the feeding zone of control sugar-agarose cubes for $512 \pm 10 \mathrm{sec}$ whereas bees exposed to the DEET treated sugar-agarose cubes were in the feeding zone for $166 \pm 45 \mathrm{sec}$ (Fig. 1A). Nerol reduced the time spent by bees in the feeding zone greater than that spent by bees in the control, isoamyl acetate, Nasonov pheromone, and DEET treatment. Bees exposed to nerol-treated cubes at $0.1 \%(\mathrm{v} / \mathrm{v})$ spent $18 \pm 3 \mathrm{sec}$ in the feeding zone (Fig. 1A). This reduction was not significantly different from that observed with geraniol and citral. Geraniol and citral reduced the time spent by bees; however, the reduction in time was not significantly different from that of Nasonov and DEET. Bees exposed to $0.1 \%$ (v/v) Nasonov, DEET, geraniol, and citral spent $175 \pm$ $49 \mathrm{sec}, 166 \pm 45 \mathrm{sec}, 64 \pm 14 \mathrm{sec}$, and $130 \pm 32 \mathrm{sec}$, respectively (Fig. 1A). Bees exposed 
to isoamyl acetate at $0.1 \%(\mathrm{v} / \mathrm{v})$ spent $417 \pm 38 \mathrm{sec}$ in the feeding zone, which was not significantly different from control treatment (Fig. 1A).

The amount of time spent by bees in the feeding zone containing sugar-agarose cubes treated with pheromonal compounds and DEET at $1 \%(\mathrm{v} / \mathrm{v})$ was observed as isoamyl acetate $($ more time spent $)<$ nasonov pheromone $=$ geraniol $=$ citral $=$ nerol $=$ DEET $($ less time spent) (Fig. 1B). Bees were recorded in the feeding zone of the untreated sugar agarose cubes (control treatment) for $507 \pm 10 \mathrm{sec}$, whereas bees exposed to DEET-treated sugar-agarose cubes were in the feeding zone for $16 \pm 4 \mathrm{sec}$ (Fig. 1B). The pheromonal compounds reduced the amount of time spent in the feeding zone by $94 \%$ compared to the control treatment. Bees exposed to Nasonov-, geraniol-, citral-, and nerol-treated sugaragarose cubes spent $32 \pm 6 \mathrm{sec}, 27 \pm 4 \mathrm{sec}, 23 \pm 3$, and $19 \pm 2 \mathrm{sec}$, respectively, and were not significantly different from the feeding time spent by bees exposed to DEET-treated sugar-agarose cubes (Fig. 1B). Bees exposed to isoamyl acetate-treated sugar-agarose cubes spent $265 \pm 48 \mathrm{sec}$ in the feeding zone, which was significantly different than the time spent by bees in the feeding zones of the control treatment (Fig. 1B).

The amount of time spent by bees in the feeding zone when exposed to $0.1 \%(\mathrm{v} / \mathrm{v})$ Nasonov, citral, isoamyl acetate, and DEET was significantly different from the time spent in the feeding zone when bees were exposed to $1 \%(\mathrm{v} / \mathrm{v})$ of those compounds (Fig. 2A, B, E, and F). The reduction in time when increasing the concentration of Nasonov, citral, isoamyl acetate, and DEET by 10 -fold was $89 \%, 88 \%, 36 \%$, and $90 \%$, respectively (Fig. 2A, B, E, and F). Increasing geraniol and nerol concentrations by 10 -fold did not significantly reduce 
the amount of time spent by bees exposed to the treated sugar-agarose cubes (Fig. 2C, and D).

\subsection{DISCUSSION}

It has been demonstrated that bees provide a significant benefit to the agricultural economy by improving crop yields by as much as $90 \%$ (McGregor 1976, Southwick and Southwick 1992, Delaplane and Mayer 2000). Pesticide exposure threatens the health of bee colonies; however, pesticide use will continue to be needed to meet the dietary needs of the world's population (vanEngelsdorp and Meixner 2010). The use of a repellent to mitigate the interaction time between foraging bees and a pesticide-treated crop has been suggested before, although, most studies on repellent discovery have been for the purpose of reducing disease transmission by biting arthropods (Atkins et al. 1975, Free et al. 1985, Katz et al. 2008). This laboratory study evaluated the efficacy of bee pheromonal compounds to deter bee foragers from a food source using video-tracking experiments. Here, it was observed that the pheromonal compounds were as effective as DEET in deterring bees from a treated food source.

This study provides evidence that Nasonov, citral, geraniol, and nerol can manipulate bee behavior by deterring the bees from a treated food source. This is not only an interesting finding, but also a surprising one, since Nasonov is used by bees as an orientation pheromone (Schmidt 1999). It is also used by beekeepers as a bee swarm attractant in boxes. Schmidt (1999) reports that bees are attracted to Nasonov five times more than other attractants used for bee swarms. Nasonov is a blend of six monoterpenes and $(E, E)$ 
- farnesol, with a number of them found to elicit behavioral changes in other organisms (Pickett et al. 1980). Nasonov consists of three main compounds: citral, geraniol, and nerol (Boch and Shearer 1964, Shearer and Boch 1966). Geraniol and citral have been shown to reduce feeding in the freshwater snail, Planobis corneus L., and geraniol has repellent activity on the parasitic mite, Varroa jacobsoni (Hoppe and Ritter 1988, Price and Berry 2008). Nerol has been found to be an alarm pheromone in the stingless bee, Trigona fulviventris (Guerin), (Johnson and Wiemer 1982) and significantly reduces the time spent by individual bees in a feeding zone. Isoamyl acetate, one of the active components of the honey bee alarm pheromone meant to attract defenders to a threat (Boch et al. 1962), was also found to reduce the amount of time that a honey bee will spend in the feeding zone in our petri dish arenas. Isoamyl acetate was also previously shown to repel foraging bees and bumble bees away from sprayed crops (Free et al. 1985). There are reports that isoamyl acetate has repellent activity at concentrations above $c a .200 \mu \mathrm{g} / \mu \mathrm{l}$ (Boch et al. 1970); however, the concentration used in this study was $c a .9 \mu \mathrm{g} / \mu \mathrm{l}$. The stationary position of the sugar-agar cube treated isoamyl acetate might have affected the attractant properties of the compound to the bees, since Free (1961) reports that the alarm pheromone is not enough to elicit a defensive behavior from the bees, but require movement of the source.

In this study, DEET was used as a positive control because it is considered to be the gold standard in arthropod repellent testing and is recommended by the WHO and EPA for evaluating repellent compounds (Lawrence et al. 2014). Additionally, it has been shown to disperse bees that are defending their colony when disturbed (Collins et al. 1996). Interestingly, DEET was found not to elicit an olfactory response using a proboscis 
extension reflex assay (Abramson et al. 2010). DEET at 1\% (v/v) reduced the amount of time an individual bee would spend in the feeding zone by 32 -fold compared to control treatment. When compared to the other compounds, Nasonov, citral, geraniol, and nerol are as effective as DEET for deterring bees from a food source. However, when the concentration of these compounds is reduced by 10 -fold, the effectiveness of all the compounds, except geraniol and nerol, is reduced. Whether a compound is a deterrent or not will depend on the concentration threshold needed for the bee to detect the compound and for that compound to elicit a response of the bee (Bernier et al. 2007). The reduction in concentration of the compounds in the food cubes would subsequently reduce the amount of detection made by the bees resulting in a less deterrent effect. Interestingly, an electroantennogram recording study found that citral elicited the strongest response in bee antennae, followed by geraniol and nerol (Williams et al. 1982). In this study, it is possible that detection of citral is still occurring at the same level as geraniol and nerol, but the response elicited is changed from deterrence to acceptance. Butler and Calam (1969) found that citral was more attractive to bees in a field assay compared to geraniol and nerol.

Overall, this study provides evidence that bee pheromonal compounds can act as deterrents for bees. The tracking method used provides an excellent technique to determine whether a compound is eliciting a deterrent effect in bees; however, repellency is a more desirable endpoint. Repellency is strictly defined as "directed movement away from a source" (Dethier et al. 1960). Following this definition, the described method is unable to discern repellency. However, with this strict definition, compounds that mask a target from an insect are excluded from being considered repellents. This led to an expanded definition 
put forth by Bernier et al. (2007) stating that "any compound that prevents an insect from reaching an intended target, that it would otherwise be attracted to if the compound were not there" is repellent. Using this expanded definition, the effects observed from pheromone treated sugar-agarose cubes suggest that the compounds are inducing repellency. This study provides evidence that pheromonal and other naturally occurring compounds can be further investigated for repellent effects in pollinators. Through use of structure-activity relationships, to drive the screening process of candidate chemistries, a highly effective compound could be discovered, and ultimately used in conjunction with a pesticide application for the protection of pollinators. 


\section{Compounds $0.1 \%$}

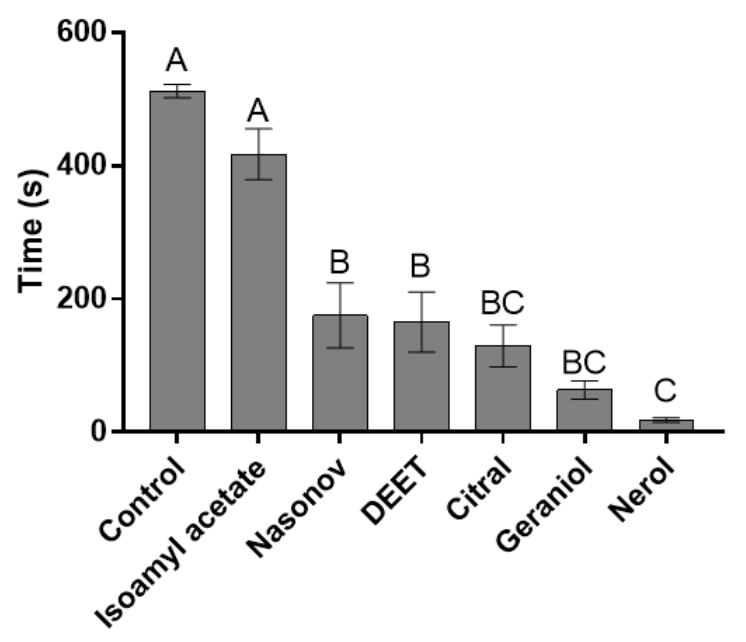

Compounds $1 \%$

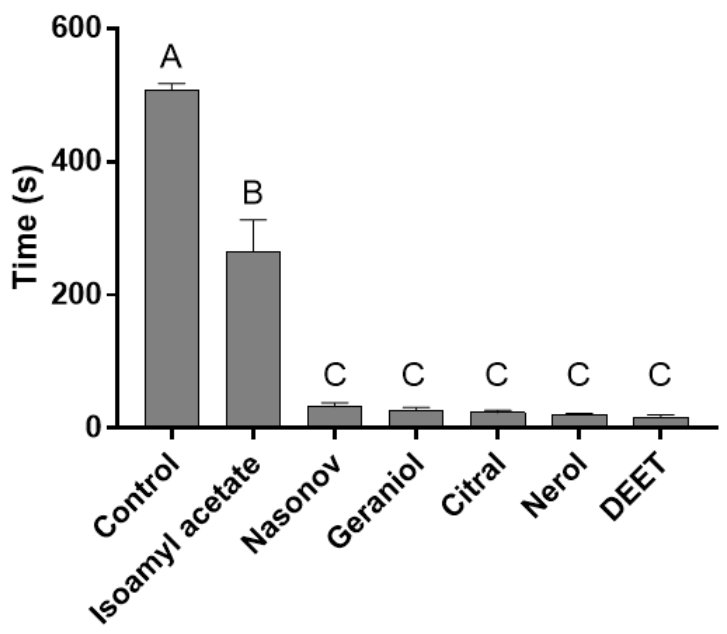

Figure 1. Efficacy of pheromonal compounds at 0.1 and $1 \%$ (v/v). Utilizing a video tracking protocol individual bees were screened in Petri dishes for behavior changes when exposed to sugaragarose cubes treated with pheromones $(0.1 \%$ or $1 \% \mathrm{v} / \mathrm{v})$. Controls were sugar-agarose cubes with no treatment. DEET treated sugar-agarose cubes were screened as a positive control. A) Compounds at $0.1 \%(\mathrm{v} / \mathrm{v})$. B) Compounds at $1 \%(\mathrm{v} / \mathrm{v})$. Vertical bars represent mean \pm SE. Different letters above bars indicate significant difference between means. One-way ANOVA with a Tukey's post hoc test $(\mathrm{P}<0.0001)$. 

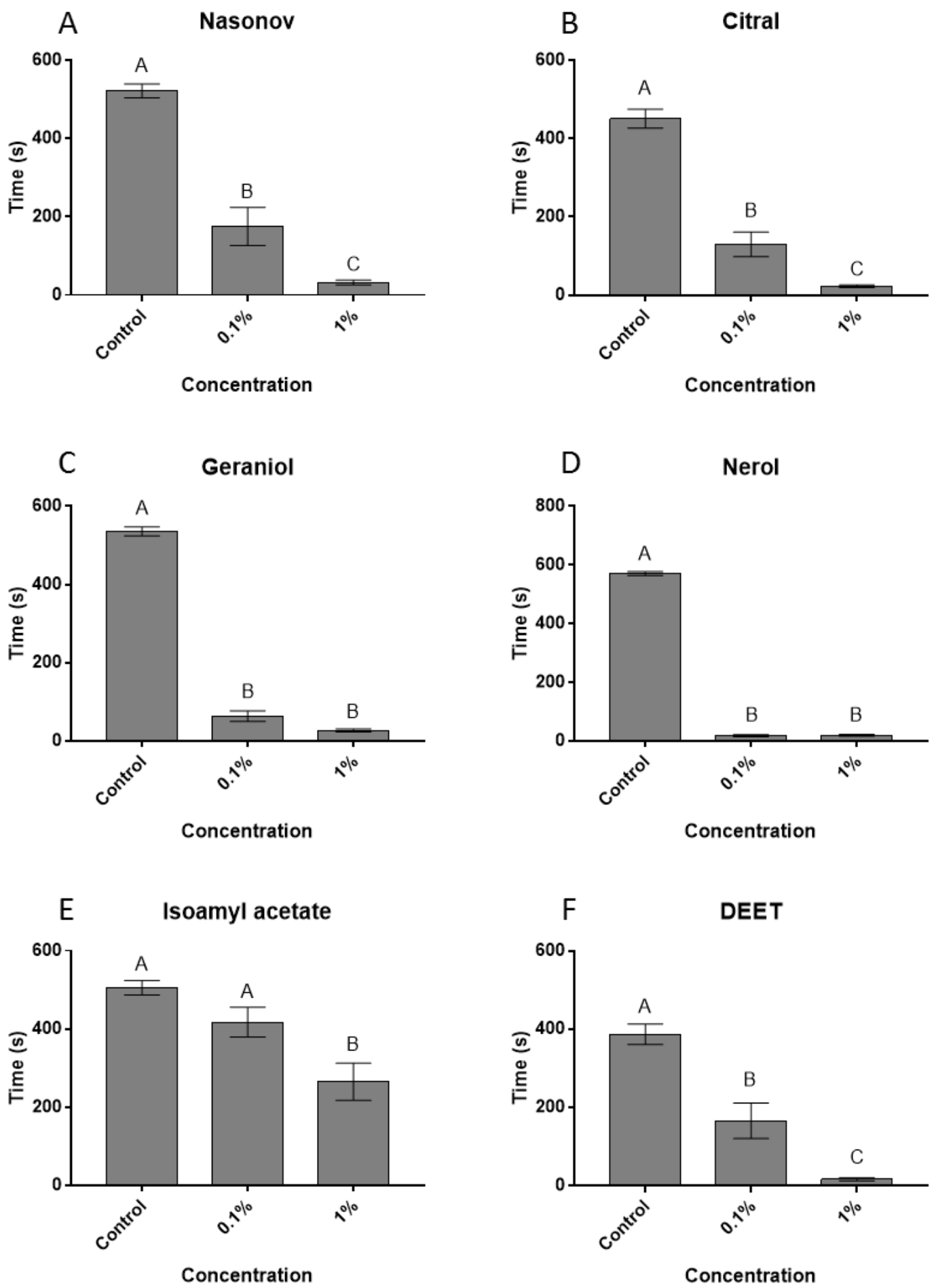
Figure 2. Effect of pheromones on the feeding behavior of honey bees. A video tracking protocol was used for individual bees in Petri dishes to monitor behavior changes when exposed to sugar-agarose cubes treated with pheromones $(0.1 \%$ or $1 \% \mathrm{v} / \mathrm{v})$. Controls were sugar-agarose cubes with no treatment. DEET treated sugar-agarose cubes were screened as a positive control. A) Nasonov pheromone. B) Citral. C) Geraniol. D) Nerol. E) Isoamyl acetate. F) DEET. Vertical bars represent mean \pm SE. Different letters above bars indicate significant difference between means. One-way ANOVA with a Tukey's post hoc test $(\mathrm{P}<0.01)$. 


\subsection{REFERENCES}

Abramson, C. I., T. Giray, T. A. Mixson, S. L. Nolf, H. Wells, A. Kence, and M. Kence. 2010. Proboscis conditioning experiments with honeybees, Apis mellifera caucasica, with butyric acid and DEET mixture as conditioned and unconditioned stimuli. J. Insect Sci. 10: 122.

Aizen, M. A., and L. D. Harder. 2009. The global stock of domesticated honey bees is growing slower than agricultural demand for pollination. Curr. Biol. 19: 915-918.

Atkins, E. L., R. L. Macdonald, and E. A. Greywood-Hale. 1975. Repellent additives to reduce pesticide hazards to honey bees : Field tests. Environ. Entomol. 4: 207-210.

Bernier, U. R., D. L. Kline, and K. H. Posey. 2007. Human emanations and related natural compounds that inhibit mosquito host-finding abilities, pp. 77-100. In Debboun, M., Frances, S.P., Strickman, D. (eds.), Insect Repellents Princ. Methods, Uses. CRC Press, Boca Raton.

Boch, R., and D. A. Shearer. 1964. Identification of nerolic and geranic acids in the Nassanoff pheromone of the honey bee. Nature. 202: 320-321.

Boch, R., D. A. Shearer, and A. Petrasovits. 1970. Efficacies of two alarm substances of the honey bee. J. Insect Physiol. 16: 17-24.

Boch, R., D. A. Shearer, and B. C. Stone. 1962. Identification of iso-aamyl acetate as an active component in the sting pheromone of the honey bee. Nature. 195: 1018-1020.

Brandt, A., A. Gorenflo, R. Siede, M. Meixner, and R. Büchler. 2016. The neonicotinoids thiacloprid, imidacloprid, and clothianidin affect the 
immunocompetence of honey bees (Apis mellifera L.). J. Insect Physiol. 86: 40-47.

Butler, C. G., and D. H. Calam. 1969. Pheromones of the honey bee-the secretion of the Nassanoff gland of the worker. J. Insect Physiol. 15: 237-244.

Christen, V., F. Mittner, and K. Fent. 2016. Molecular effects of neonicotinoids in honey bees (Apis mellifera). Environ. Sci. Technol. 50: 4071-4081.

Cizelj, I., G. Glavan, J. Božič, I. Oven, V. Mrak, and M. Narat. 2016. Prochloraz and coumaphos induce different gene expression patterns in three developmental stages of the Carniolan honey bee (Apis mellifera carnica Pollmann). Pestic. Biochem. Physiol. 128: 68-75.

Collins, A. M., W. L. Rubink, J. I. Cuadriello Aguilar, and R. L. Hellmich Ii. 1996. Use of insect repellents for dispersing defending honey bees (Hymenoptera: Apidae). J. Econ. Entomol. 89: 608-613.

Cresswell, J. E. 2011. A meta-analysis of experiments testing the effects of a neonicotinoid insecticide (imidacloprid) on honey bees. Ecotoxicology. 20: 149-157.

Delaplane, K. S., and D. F. Mayer. 2000. Crop pollination by bees. CABI, New York.

Dethier, V. G., B. L. Browne, and C. N. Smith. 1960. The designation of chemicals in terms of the responses they elicit from insects. J. Econ. Entomol. 53: 134-136.

Fairbrother, A., J. Purdy, T. Anderson, and R. Fell. 2014. Risks of neonicotinoid insecticides to honeybees. Environ. Toxicol. Chem. 33: 719-731.

Free, J. B., J. A. Pickett, A. W. Ferguson, J. R. Simpkins, and M. C. Smith. 1985. Repelling foraging honeybees with alarm pheromones. J. Agric. Sci. 105: 255. 
Garcia, M. D. G., S. U. Duque, A. B. L. Fernandez, A. Sosa, and A. R. FernandezAlba. 2017. Multiresidue method for trace pesticide analysis in honeybee wax comb by GC-QqQ-MS. Talanta. 163: 54-64.

Hoppe, H., and W. Ritter. 1988. The influence of the Nasonov pheromone on the recognition of house bees and foragers by Varroa jacobsoni. Apidologie. 19: 165172.

Johnson, L. K., and D. F. Wiemer. 1982. Nerol: An alarm substance of the stingless bee, Trigona fulviventris (Hymenoptera: Apidae). J. Chem. Ecol. 8: 1167-1181.

Johnson, R. M., M. D. Ellis, C. A. Mullin, and M. Frazier. 2010. Pesticides and honey bee toxicity - USA. Apidologie. 41: 312-331.

Katz, T. M., J. H. Miller, and A. A. Hebert. 2008. Insect repellents: historical perspectives and new developments. J. Am. Acad. Dermatol. 58: 865-871.

Larson, N., and T. Anderson. 2017. Video tracking protocol to screen deterrent chemistries for honey bees. J. Vis. Exp. (In Press): e55603.

Lawrence, K. L., N. L. Achee, U. R. Bernier, K. D. Mundal, and J. P. Benante. 2014. Field evaluations of topical arthropod repellents in North, Central, and South America. J. Med. Entomol. 51: 980-988.

McGregor, S. E. 1976. Insect pollination of cultivated crop plants: Agriculture handbook, USDA. USDA-ARS, Washington D.C.

Mullin, C. A., M. Frazier, J. L. Frazier, S. Ashcraft, R. Simonds, D. VanEngelsdorp, and J. S. Pettis. 2010. High Levels of miticides and agrochemicals in North American 
apiaries: Implications for honey bee health. PLoS One. 5: e9754.

Pickett, J. A., I. H. Williams, A. P. Martin, and M. C. Smith. 1980. Nasonov pheromone of the honey bee, Apis mellifera L. (Hymenoptera: Apidae) Part I. Chemical Characterization. J. Chem. Ecol. 6: 425-434.

Price, D. N., and M. S. Berry. 2008. Neurophysiological effects of naturally occurring defensive compounds on the freshwater snail Planorbis corneus: Comparison with effects in insects. J. Chem. Ecol. 34: 994-1004.

Sanchez-Bayo, F., and K. Goka. 2014. Pesticide residues and bees - A risk assessment. PLoS One. 9: 1-16.

Schmidt, J. O. 1999. Attractant or pheromone: The case of Nasonov secretion and honeybee swarms. J. Chem. Ecol. 25: 2051-2056.

Seitz, N., K. S. Traynor, N. Steinhauer, K. Rennich, M. E. Wilson, J. D. Ellis, R. Rose, D. R. Tarpy, R. R. Sagili, D. M. Caron, K. S. Delaplane, J. Rangel, K. Lee, K. Baylis, J. T. Wilkes, J. A. Skinner, J. Pettis, and D. VanEngelsdorp. 2016. A national survey of managed honey bee 2014-2015 annual colony losses in the USA. J. Apic. Res. 54: 292-304.

Shearer, D. A., and R. Boch. 1965. 2-Heptanone in the mandibular gland secretion of the honey-bee. Nature. 202: 320-32.

Shearer, D. A., and R. Boch. 1966. Citral in the Nassanoff pheromone. J. Insect Physiol. 12: $1513-1521$

Smith, K. M., E. H. Loh, M. K. Rostal, C. M. Zambrana-Torrelio, L. Mendiola, and 
P. Daszak. 2013. Pathogens, pests, and economics: Drivers of honey bee colony declines and losses. Ecohealth. 10: 434-445.

Southwick, E. E., and L. Southwick. 1992. Estimating the economic value of honey bees (Hymenoptera: Apidae) as agricultural pollinators in the United States. J. Econ. Entomol. 85: 621-633.

Taylor, K. S., G. D. Waller, and L. A. Crowder. 1987. Impairment of a classical conditioned response of the honey bee (Apis mellifera L.) by sublethal doses of synthetic pyrethroid insecticides. Apidologie. 18: 243-252.

Teeters, B. S., R. M. Johnson, M. D. Ellis, and B. D. Siegfried. 2012. Using videotracking to assess sublethal effects of pesticides on honey bees (Apis mellifera L.). Environ. Toxicol. Chem. 31: 1349-1354.

Thompson, H. M. 2010. Risk assessment for honey bees and pesticides-recent developments and "new issues." Pest Manag. Sci. 66: 1157-1162.

Tison, L., M. L. Hahn, S. Holtz, A. Robner, U. Greggers, G. Bischoff, and R. Menzel. 2016. Honey bees' behavior is impaired by chronic exposure to the neonicotinoid thiacloprid in the field. Environ. Sci. Technol. 50: 7218-7227.

(USDA-NASS) United States Department of Agriculture National Agriculture Statistics Service. 2017. Honey. USDA, Wahington, DC.

Vallet, A., P. Cassier, and Y. Lensky. 1991. Ontogeny of the fine structure of the honeybee (Apis mellifera L.) workers and the pheromonal activity of 2-heptanone. J. Insect Physiol. 37: 789-804. 
vanEngelsdorp, D., and M. D. Meixner. 2010. A historical review of managed honey bee populations in Europe and the United States and the factors that may affect them. J. Invertebr. Pathol. 103: S80-S95.

Williams, I., J. Pickett, and A. Martin. 1982. Nasonov pheromone of the honey bee, Apis mellifera L. (Hymenoptera: Apidae). Comp. Gen. Pharmacol. 8: 567-574.

Williamson, S. M., and G. A. Wright. 2013. Exposure to multiple cholinergic pesticides impairs olfactory learning and memory in honeybees. J. Exp. Biol. 216: 1799-1807. 


\section{CHAPTER 5: EFFECT OF HETEROCYCLIC AMINES ON THE FEEDING BEHA VIOR OF HONEY BEES}

\subsection{ABSTRACT}

Pesticides are a risk to honey bee colony health. Reduction in the vitality of colonies increases the probability that beekeeping operations will lose significant numbers of managed hives. This in turn increases the beekeeper's operational costs. Reducing the interaction time between foraging bees and pesticide treated crops will potentially minimize the number and amount of pesticide residue being brought back to their colonies. One way to reduce interaction times is to use repellents to keep foraging bees away from the treated crops. A group of compounds called heterocyclic amines (HCAs) have been screened in this study for their effect on feeding behavior in bees. Individual bees were placed into Petri dishes that contained control and treated sugar-agarose cubes. Videotracking software was used to monitor the positions of the bees for $10 \mathrm{~min}$ in relation to a feeding zone. It was found that $15 \mathrm{HCAs}$ reduced the time spent in the feeding zone by bees by up to $94 \%$ compared to controls. Piperidine was the most efficacious HCA reducing the time spent by bees to $31 \mathrm{sec}$ compared to controls that spent $505 \mathrm{sec}$ within feeding zones. Altering the structures of piperidine, pyrrole, piperazine, and pyrrolidine had varying effects on the effectiveness of the compounds. Adding functional groups to these ring structures was shown to increase effectiveness or decrease effectiveness. The gold standard in arthropod repellent testing, DEET, was shown to reduce the time spent in the feeding zones by $97 \%$ to just 16 sec.

\subsection{INTRODUCTION}


Pesticide exposure can have deleterious effects on honey bee colonies (Mullin et al. 2010, vanEngelsdorp and Meixner 2010). A recent risk assessment by Sanchez-Bayo and Goka (2014) found that 161 pesticide residues have been identified in bee hives across the world. Insecticides comprised $52 \%$ of the residues. Fungicides were the second largest group found at $25 \%$, followed by herbicides at $17 \%$, and finally acaricides at $6 \%$. Pesticide exposure in bees result in several different effects that range from acute toxicity to sublethal effects including paralysis, disorientation, and behavioral changes (vanEngelsdorp and Meixner 2010).

The two most common pesticides found in bee colonies are coumaphos and tau-fluvalinate, the active miticide ingredients in Checkmite $+{ }^{\circledR}$ and Apistan ${ }^{\circledR}$ that are used to control the Varroa destructor (Anderson and Trueman) mite (Mullin et al. 2010, Sanchez-Bayo and Goka 2014, Garcia et al. 2017). Coumaphos and tau-fluvalinate have been found to impair olfactory learning (Taylor et al. 1987, Williamson and Wright 2013). Coumaphos has additionally been found to downregulate immune genes in prepupal bees (Cizelj et al. 2016). Teeters et al. (2012) found that tau-fluvalinate decreased locomotor activity in bees. It has also been shown to reduce the size of queens (Haarmann et al. 2002). More recently, neonicotinoids have come under increased scrutiny since becoming the most widely used insecticides in the world (Goulson 2013). Studies have found that neonicotinoids have detrimental effects on bee navigation, motivation to forage, and social communication (Tison et al. 2016). They additionally negatively affect bee immunocompetence, gland and respiratory system development, reduce the survival of queens with Nosema infection, and reduce sperm viability in queens (Hatjina et al. 2013, Brandt et al. 2016, Chaimanee et al. 
2016, Christen et al. 2016, Dussaubat et al. 2016). The fungicide chlorothalonil has been shown to alter the compilation of bee gut flora, potentially altering functionality resulting in decreased vigor (Kakumanu et al. 2016). Fungicides have also been found to increase bee susceptibility to Nosema infection (Pettis et al. 2013) and synergize the toxicity of taufluvalinate and coumaphos (Johnson et al. 2013, Pettis et al. 2013). The herbicide atrazine has been found to induce oxidative stress in bees through the reduction of antioxidant activity (Williams 2016). It is likely that pesticides will continue to be used in the future, as achieving high yields in modern agriculture requires their use (vanEngelsdorp and Meixner 2010). In order to mitigate honey bee exposure to harmful compounds, new regulations and methods need to be developed and implemented to protect the agricultural benefit of pollinators (Mullin et al. 2010). One potential method, that has been alluded to since 1900 , is to provide protection by utilizing repellents during pesticide spraying to keep pollinators away from the treated crops (Callbreath 1900, Atkins et al. 1975, Free et al. 1985).

Historically, insect repellents (IRs) have been used to prevent the spread of deadly diseases by arthropod vectors (Katz et al. 2008). DEET is the most widely used and most efficacious IR to be developed in the past 60 years (Katz et al. 2008, Dickens and Bohbot 2013). Before World War II, and the introduction of DEET, four IRs were available for consumer use, including citronella oil, dialkyl phthalate, Indalone ${ }^{\circledR}$, and Rutgers 612 (Brown and Hebert 1997). DEET has become an important IR and is the gold standard for topically applied arthropod repellent testing. It is recommended by the World Health Organization (WHO) and the United States Environmental Protection Agency (EPA) as a positive 
control for novel repellent screening (Moore and Debboun 2007, Lawrence et al. 2014). However, the safety of DEET is a highly debated topic, which has led to the discovery of safer repellents (Diaz 2016). During the 1970s, hundreds of synthetic compounds related to piperidine were developed, and are currently being re-evaluated for their potential use as repellents (Moore and Debboun 2007).

Piperidine is a heterocyclic amine (HCA) consisting of a six-membered ring structure with five carbons and one nitrogen. It was isolated from black pepper in 1852 (Warnhoff 1998). Piperidine analogs have been found to repel several types of arthropods. The analog 1-(3cyclohexen-1-ylcarbonyl)-2-methylpiperidine was shown to repel the lone star tick, Amblyomma americanum, away from humans significantly longer than DEET, and one of its stereoisomers has been reported as an effective repellent for mosquitoes and chigger mites (Solberg et al. 1995, Klun et al. 2001). Schreck et al. (1995) found that 1-(3cyclohexenyl-carbonyl) piperidine provided a longer protection time against the lone star tick compared to DEET. The EPA currently recognizes seven active ingredients for topically applied insect repellents, including Picaridin, a piperidine compound shown to be a highly effective tick repellent (Carroll et al. 2008, "United States Environmental Protection Agency" 2017). Bernier et al. (2012) report the use of HCAs as a novel method for repelling mosquitoes. These reported HCAs were observed to cause hyposmic or anosmic effects with the mosquitoes and, thus, inhibit their ability to locate a host. Based on the above findings, I hypothesized that exposure to HCAs within a food source would effectively repel bees from that food source and, ultimately, provide a method of protection from pesticide-treated crops. In this study, we impregnated sugar-agarose cubes with 
piperidine and 27 additional HCAs (final concentrations of $1 \%(\mathrm{v} / \mathrm{v})$ ) and utilizing a video tracking protocol recorded the position of bees in Petri dish arenas over a 10-min period. It was observed that bees spent less time on food cubes infused with HCAs compared to control treatments suggesting that these compounds are deterring bees from the food source or possibly masking the food source from the bees.

\subsection{MATERIALS AND METHODS}

\subsubsection{Honey bees}

Bees were maintained in an apiary at Virginia Tech's Prices Fork Research Facility (Blacksburg, VA) using standard beekeeping practices. Bee workers were collected from frames located in the uppermost super of a colony to ensure that a large number of newly emerged and nurse bees were not collected prior to testing the repellent compounds. The collected worker bees were maintained in an incubator at $32{ }^{\circ} \mathrm{C}$ with $c a .70 \%$ relative

humidity and starved overnight prior to the compound exposures and video-tracking recordings.

\subsubsection{Heterocyclic amines and DEET}

The heterocyclic amines (Figs. 1 and 2) were purchased from Sigma-Aldrich Chemical Co. (St. Louis, MO). OFF! Deep Woods® Sportsmen was used at the source for DEET (98\% active ingredient; $N, N$-Diethyl-meta-toluamide) and was purchased from a local store.

\subsubsection{Video-tracking software and recordings}


A Basler acA-1300-60gm camera and EthoVision ${ }^{\circledR}$ XT video recording software was used for the recordings (Noldus, Leesburg, VA). A light box was used to illuminate the assay arena with Cryon LED lights (Model HTP904E, Chatsworth, CA) set to the red spectrum to avoid bee light bias. The light box was and camera system was covered with a black plastic sheet to eliminate ambient light.

A video-tracking protocol described by Larson and Anderson (2017) was used to evaluate the effects of the HCAs and DEET on the feeding behavior of bees. Briefly, sugar-agarose cubes were prepared by dissolving $8 \mathrm{~g}$ of sugar into $20 \mathrm{ml}$ deionized $\mathrm{H}_{2} \mathrm{O}$ followed by the addition of $170 \mathrm{mg}$ agarose. The sugar-agarose mixture was heated and the solution was poured into $1.5 \times 1.5 \times 0.3 \mathrm{~cm}$ molds. The HCAs and DEET were added to the sugaragarose solution, at the desired concentrations, prior to pouring the solution into the molds. A total of 8 control sugar-agarose cubes (no HCA or DEET) and 8 compound HCA or DEET sugar-agarose cubes were placed into the centers of $9 \mathrm{~cm}$ diameter Petri dishes (i.e., feeding zone). The Petri dishes were positioned on top of the light box in a 4 x 4 pattern and individual bees were placed into each of the Petri dishes. The EthoVision® videotracking recordings were conducted with bees exposed to the control and compound sugaragarose cubes for 10 min followed by another set of recordings with new bees exposed to the same cubes for another $10 \mathrm{~min}$. Each video-tracking recording was conducted in duplicate. The time spent by the bees in the feeding zones was calculated by the EthoVision $®$ video-tracking software and the data were statistically analyzed using a oneway ANOVA with a Tukey's post hoc test to determine significantly different means in GraphPad Prism (La Jolla, CA) software. 


\subsection{RESULTS}

The amount of time spent by bees in the feeding zones with HCA and DEET treated sugaragarose cubes was observed as piperazine $($ more time spent $)=$ pyrrolidine $>$ pyrrole $=$ piperidine $=$ DEET (less time spent) $($ Fig. 3A). Bees were recorded in the feeding zone of the control sugar-agarose cubes for $505 \pm 5 \mathrm{sec}$ whereas bees exposed to the DEET treated sugar-agarose cubes were in the feeding zone for $16 \pm 4 \mathrm{sec}$ (Fig. 3A). Piperidine and pyrrole reduced the time bees spent in the feeding zone greater than that of the other HCAs. Bees exposed to piperidine treated cubes were in the feeding zone for $31 \pm 10 \mathrm{sec}$ and bees exposed to pyrrole treated cubes spent $74 \pm 12 \mathrm{sec}$ in the feeding zone (Fig. 3A). Pyrrolidine and piperazine had a lesser effect at reducing the time spent by bees in the feeding zone. Bees exposed to pyrrolidine treated cubes were in the feeding zone for 243 $\pm 35 \mathrm{sec}$ whereas bees exposed to piperazine treated cubes spent $262 \pm 45 \mathrm{sec}$ in the feeding zone (Fig. 3A).

Altering the structure of pyrrole reduces the observed time spent in the feeding zone by the bees (Fig. 3B). The amount of time spent by bees in the feeding zones with substituted pyrrole treated sugar-agarose cubes was observed as pyrrole at 2-methyl-2-imidazoline (more time spent) $>N$-methylpyrrole $>$ imidazole $>$ methyl imidazole $=$ pyrrole $>$ DEET (less time spent) (Fig. 3B). Bees exposed to methyl imidazole treated cubes spent $101 \pm$ $17 \mathrm{sec}$ in the feeding zone, which was $27 \mathrm{sec}$ longer than the feeding zone time spent by bees exposed to pyrrole treated cubes (Fig. 3B). Similarly, bees exposed to imidazole treated cubes were present in the feeding zone for $187 \pm 45 \mathrm{sec}$, which is $113 \mathrm{sec}$ longer 
than that of bees exposed to pyrrole treated cubes (Fig. 3B). However, there is no significant difference between the time bees spent in the feeding zone with methyl imidazole treated cubes compared to those with imidazole treated cubes (Fig. 3B). Interestingly, $N$-methylpyrrole significantly increases the feeding zone time spent by the bees compared to bees exposed to pyrrole treated cubes. Bees exposed to $N$-methylpyrrole treated cubes spent $282 \pm 43 \mathrm{~s}$ in the feeding zone (Fig. 3B). The feeding zone time spent by bees exposed to 2-methyl-2-imidazoline treated cubes increased to $425 \pm 45 \mathrm{sec}$, which is comparable to bees provided untreated sugar-agarose cubes (Fig. 3B).

Altering the structure of piperidine also decreases the observed time spent in the feeding zone by the bees (Fig. 3C). The amount of time spent by bees in the feeding zones with substituted piperidine treated sugar-agarose cubes was observed as 4-methylmorpholine (more time spent) $>$ thiomorpholine $>4$-methylpiperidine $=1$-methylpiperidine $=$ piperidine $=$ DEET (less time spent) $($ Fig. 3C). Bees exposed to 1-methylpiperidine, 4methylpiperidine, and thiomorpholine treated cubes spent $42 \pm 8 \mathrm{sec}, 62 \pm 12 \mathrm{sec}$, and 124 $\pm 14 \mathrm{sec}$ in the feeding zone, respectively, and were not significantly different from the feeding zone time spent by bees exposed to either piperidine or DEET treated cubes (Fig. 3C). In addition, 4-methylmorpholine was the least effective substituted piperidine. Bees exposed to 4-methylmorpholine treated cubes were present in the feeding zone for $301 \pm$ $33 \mathrm{sec}$, which is $270 \mathrm{sec}$ longer than bees exposed to piperidine treated agarose cubes (Fig. $3 \mathrm{C})$. 
The amount of time spent by bees in the feeding zones with substituted pyrrolidine treated sugar-agarose cubes was observed as 1-methylpyrrolidine (more time spent) > 1ethylpyrrolidine $=1$-methylpyrrolidine $=$ DEET (less time spent) $($ Fig. 3D). Bees exposed to 1-ethylpyrrolidine and 1-methylpyrrolidine treated cubes spent $73 \pm 14 \mathrm{sec}$ and $76 \pm 19$ sec in the feeding zone, respectively, which was significantly less than bees exposed to pyrrolidine treated cubes, but not bees exposed to DEET treated cubes (Fig. 3D).

The amount of time spent in the feeding zones with substituted piperazine treated sugaragarose cubes was observed as 2,6-dimethylpiperazine (more time spent) > 1-amino-4methylpiperazine $>$ 1,4-bis $(2$-hydroxyethyl $)$ piperazine $=$ piperazine $=1$-methylpiperazine $=2$-methylpiperazine $>1$-methylhomopiperzine $>1$-boc-piperazine $>$ ethyl 1 piperazinecarboxylate $>$ trans-2,5-diemthylpiperazine $=1-(2$-pyridyl $)$ piperazine $=1-4-$ dimethylpiperazine $>1$-acetylpiperazine $=1$-ethylpiperazine $=1-(2$-methoxyphenyl $)$ piperazine $=$ phenylpiperazine $>$ DEET (less time spent) $($ Fig. 4A). Bees exposed to phenylpiperazine, 1-(2-methoxyphenyl) piperazine, 1-ethylpiperazine, and 1acetylpiperazine treated cubes spent $54 \pm 9 \mathrm{sec}, 59 \pm 12 \mathrm{sec}, 67 \pm 18 \mathrm{sec}$, and $92 \pm 19 \mathrm{sec}$ in the feeding zone, respectively, which was significantly less than bees exposed to piperazine treated cubes, but not bees exposed to DEET treated cubes (Fig. 4A). Bees exposed to 1-4-dimethylpiperazine, 1-(2-pyridyl) piperazine, trans-2,5diemthylpiperazine, and ethyl 1-piperazinecarboxylate treated cubes spent $103 \pm 25 \mathrm{sec}$, $111 \pm 13 \mathrm{sec}, 115 \pm 32 \mathrm{sec}$, and $156 \pm 23 \mathrm{sec}$ in the feeding zone, respectively, which was not significantly different from bees exposed to piperazine or DEET treated cubes (Fig. 4A). Bees exposed to 1-boc-piperazine, 1-methylhomopiperzine, 2-methylpiperazine, and 
1-methylpiperazine treated cubes spent $174 \pm 32 \mathrm{sec}, 236 \pm 57 \mathrm{sec}, 253 \pm 38 \mathrm{sec}$, and 254 $\pm 36 \mathrm{sec}$ in the feeding zone, respectively, which was not significantly different from bees exposed to piperazine treated cubes, but was significantly greater than bees exposed to DEET treated cubes (Fig. 4A). Bees exposed to 1,4-bis(2-hydroxyethyl) piperazine, 1amino-4-methylpiperazine, and 2,6-dimethylpiperazine treated cubes spent $265 \pm 37 \mathrm{sec}$, $281 \pm 45 \mathrm{sec}$, and $334 \pm 46 \mathrm{sec}$ in the feeding zone, respectively, which was significantly greater than bees exposed to DEET treated cubes, but not significantly different from piperazine treated cubes (Fig. 4A).

In total, there were $15 \mathrm{HCAs}$ that were as effective as DEET in deterring bees from food cubes (Fig. 4B). There was at least one compound from each parental HCA structure that had effects that were not significantly different from DEET. The strongest of these compounds was piperidine with an average time of $31 \pm 10 \mathrm{sec}$ while the weakest was also a piperidine analog, thiomorpholine, with an average time of $124 \pm 14 \mathrm{sec}$.

\subsection{DISCUSSION}

The use of a repellent in parallel with pesticide applications has the potential to reduce pollinator exposure times to pesticide exposures (Atkins et al. 1975, Free et al. 1985). The majority of insect repellency studies have been focused on personal bite protection from disease vectoring arthropods (Katz et al. 2008). However, there have been a few studies reporting that pollinators can be repelled from areas that are sprayed with a chemical

compound (Atkins et al. 1975, Free et al. 1985, Collins et al. 1996). The present study evaluates the spatial effect of HCAs on bees relative to a food source using a video tracking 
protocol. Here, it was observed that HCA treated sugar-agarose cubes deters bees from a food source similar to that observed with DEET.

DEET is the gold standard for insect repellent testing (Lawrence et al. 2014) and was used as a positive control for this study. A total of 7 piperazines, 4 piperidines, 2 pyrrolidines, and 2 pyrroles were observed to provide effective deterrence of bees from a food source comparable to DEET. This is not surprising because several of the piperidine compounds have been shown to be highly effective at repelling mosquitoes, chigger mites, and ticks (Schreck et al. 1995, Solberg et al. 1995, Klun et al. 2001). The EPA additionally recognizes the piperidine analog Picaridin as personal repellent with similar efficacy as DEET for repelling mosquitoes (Frances et al. 2004, "United States Environmental Protection Agency" 2017).

The efficacy of the HCA compounds was dependent on the substitutions that were placed onto the parent ring structure. For pyrrolidine, efficacy was significantly increased with the additions of a methyl or ethyl group to the ring structure. Additions to the ring structure of piperazine also increased the parent compound efficacy. Both pyrrole and piperidine lose efficacy when substitutions are added to the ring structures. Taking these changes into consideration can aid in development of more effective repellent compounds. In pesticide development and discovery, there are several strategies that are used to increase the efficacy of lead compounds. One method that would be useful here would be to develop a structure activity relationship. This allows for the comparison of the molecular structure of the compounds to the physio-chemical and biological responses (Das 2016). With a 
comparative analysis of these compounds, a more effective repellent could be found. Additionally, efficacy could be improved by increasing the concentration of the compounds in the sugar-agarose cubes or changing the food source in to which the compounds are infused. The volatility of compounds can have a significant effect on the detection of it by insects (Bernier et al. 2007). Compounds with high vapor pressure will have lower volatility resulting in a reduced concentration of compound within the headspace of the area sprayed, while reducing the detectability of the compound at a distance. (Bernier et al. 2007). Increasing the concentration of the compounds or changing the food source could increase the concentration of the compounds within the head space of the sprayed area, which could increase efficacy.

Overall, this study provides evidence that the HCAs deter individual bees from food sources. The method that is utilized here to record bee behavior is an excellent way to determine if a compound has a deterrent effect. However, this method lacks the ability to clearly discern repellency as the Petri dish arena is too small to view movement away from the chemical source. A strict definition of repellency states that an insect will make direct movement away from the chemical source (Dethier et al. 1960). Bernier et al. (2007) described repellency as a compound that will prevent an insect from reaching a target that it would otherwise find if the compound were not present. Using this definition, an argument could be made that these compounds are repelling bees from the food cubes. However, the question of how the bees are detecting these compounds remains. The success of the HCAs warrant further study into the detection method used and production of structure activity relationships to develop more effective repellents. 


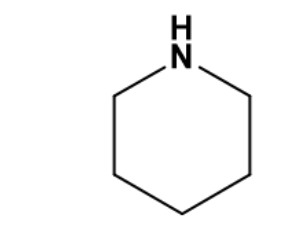

Piperidine

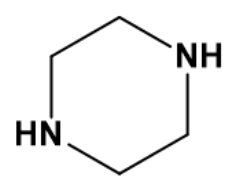

Piperazine

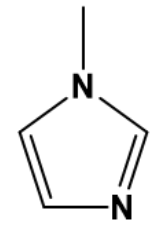

Methylimidazole

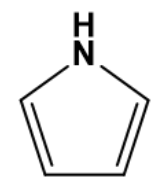

Pyrrole

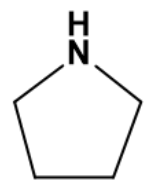

Pyrrolidine<smiles>Cn1cccc1</smiles>

N-methylpyrrole

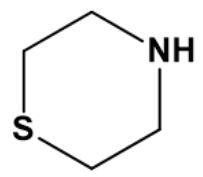

Thiomorpholine

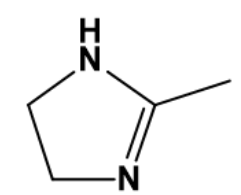

2-Methyl-2-Imidazoline

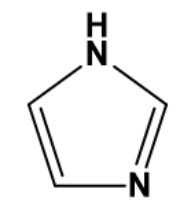

Imidazole<smiles>CC1CCNCC1</smiles>

4-Methylpiperidine

\section{4-Methylmorpholine}

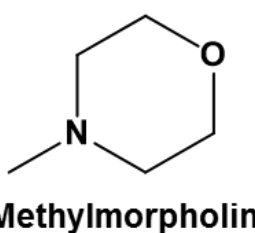

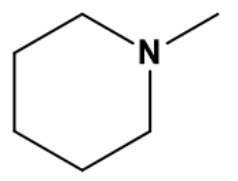

1-Methylpiperidine<smiles>CCN(CC)C(=O)c1cccc(C)c1</smiles>

$N, N$-diethyl-3-methylbenzamide

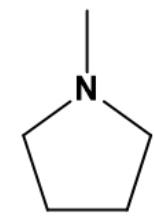

1-Methylpyrrolidine

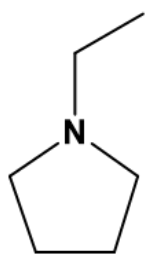

1-Ethylpyrrolidine

Figure 1. Chemical structures of the heterocyclic amines and DEET. Parent compounds: piperidine, piperazine, pyrrole, and pyrrolidine. Substituted pyrroles: $\mathrm{N}$-methylpyrrole, methylimidazole, 2-methyl-2-imidazoline, and imidazole. Substituted piperidines: 4-methylpiperidine, thiomorpholine, 4-methylmorpholine, and 1-methylpiperidine. Substituted pyrrolidines: 1-methylpyrroldine and 1ethylpyrrolidine. DEET: $N, N$-diethyl-3-methylbenzamide 


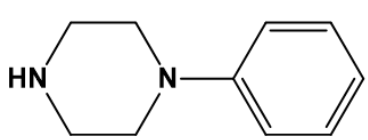

1-Phenylpiperazine

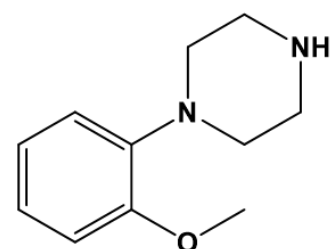

(2-Methoxyphenyl)piperazine

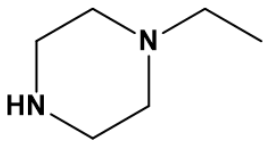

1-Ethylpiperazine<smiles>CC(=O)N1CCNCC1</smiles>

1-Acetylpiperazine

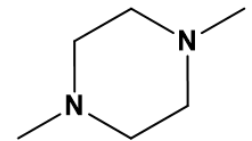

1,4-Dimethylpiperazine

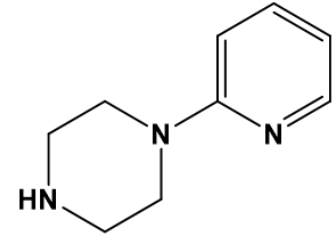

1-(2-Pyridyl)piperazine

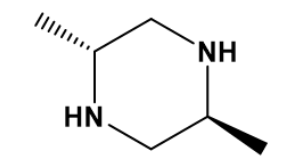

Trans-2,5-dimethylpiperazine

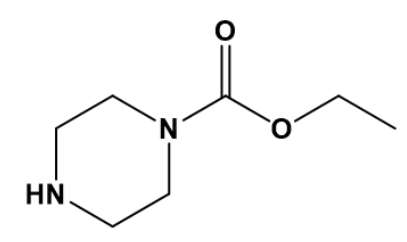

Ethyl 1-piperazinecarboxylate

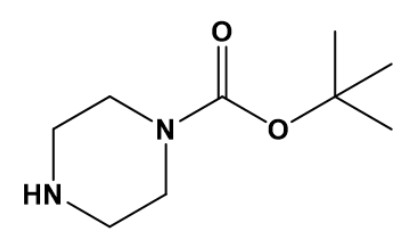

1-Boc-piperazine

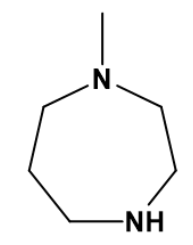

1-Methylhomopiperazine

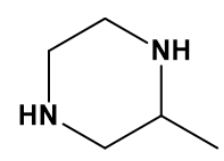

2-Methylpiperazine

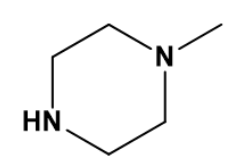

1-Methylpiperazine

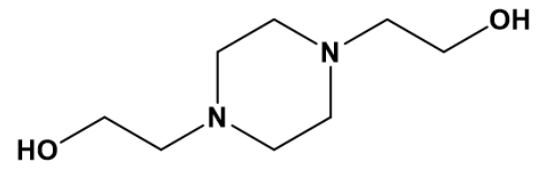

1,4-Bis(2-hydroxyethyl)piperazine

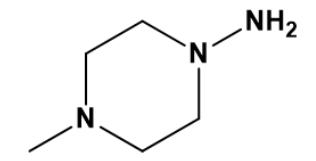

1-Amino-4-methylpiperazine

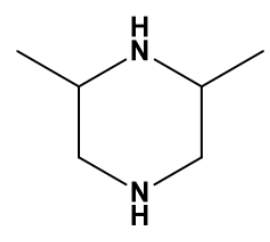

2,6-Dimethylpiperazine

Figure 2. Chemical structure of substituted piperazines 

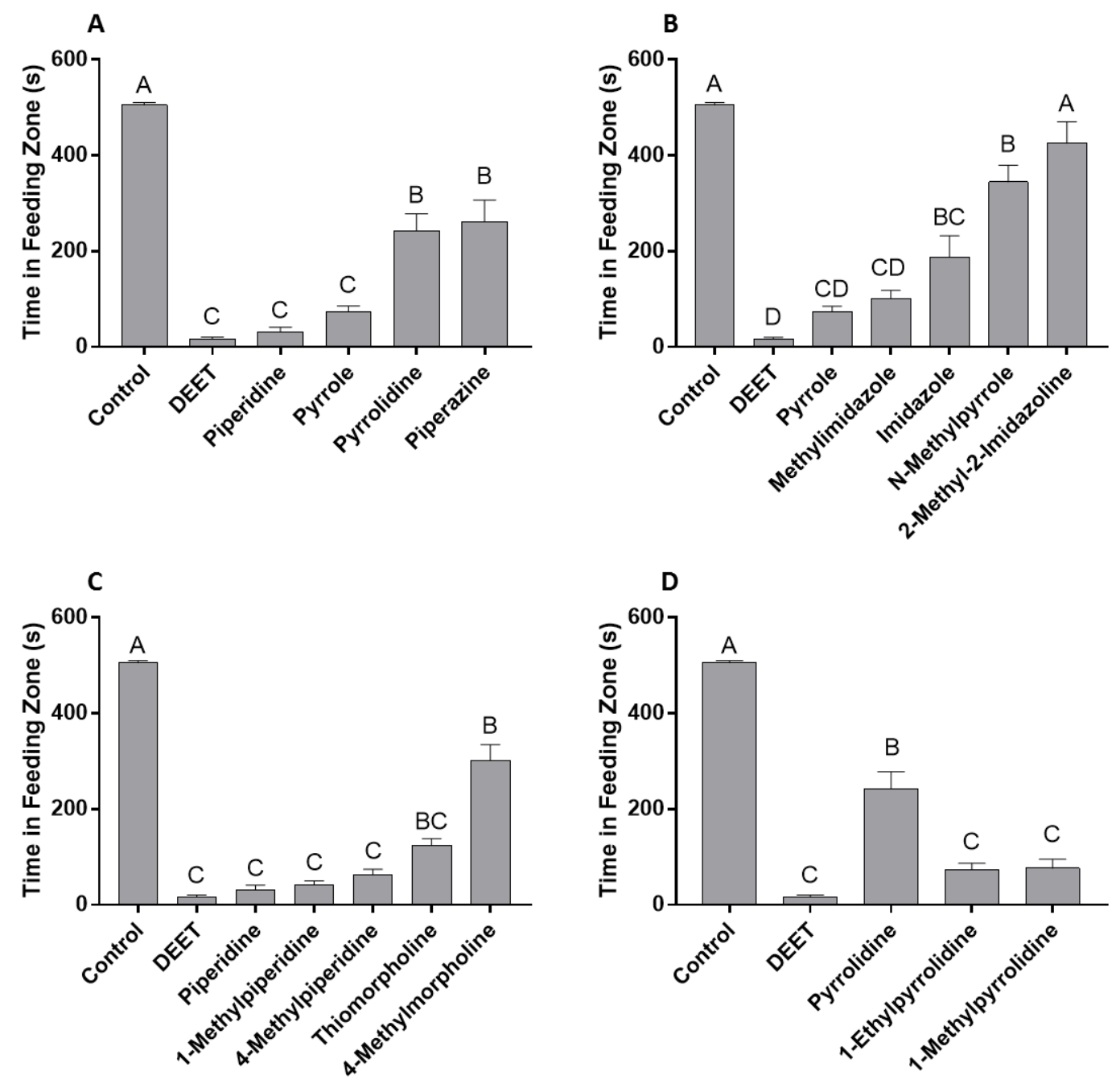

Figure 3. Effect of heterocyclic amines (HCAs) on the feeding behavior of honey bees. Utilizing a video tracking protocol individual bees were screened in Petri dishes for behavior changes when exposed to sugar-agarose cubes treated with HCAs (1\% v/v). Controls were sugar-agarose cubes with no treatment. DEET treated sugar-agarose cubes were screened as a positive control. A) HCA parent compounds. B) Substituted pyrroles. C) Substituted piperidines. D) Substituted pyrrolidines. Vertical bars represent mean \pm SE. Different letters above bars indicate significant difference between means. One-way ANOVA with a Tukey's post hoc test $(\mathrm{P}<0.05)$. 

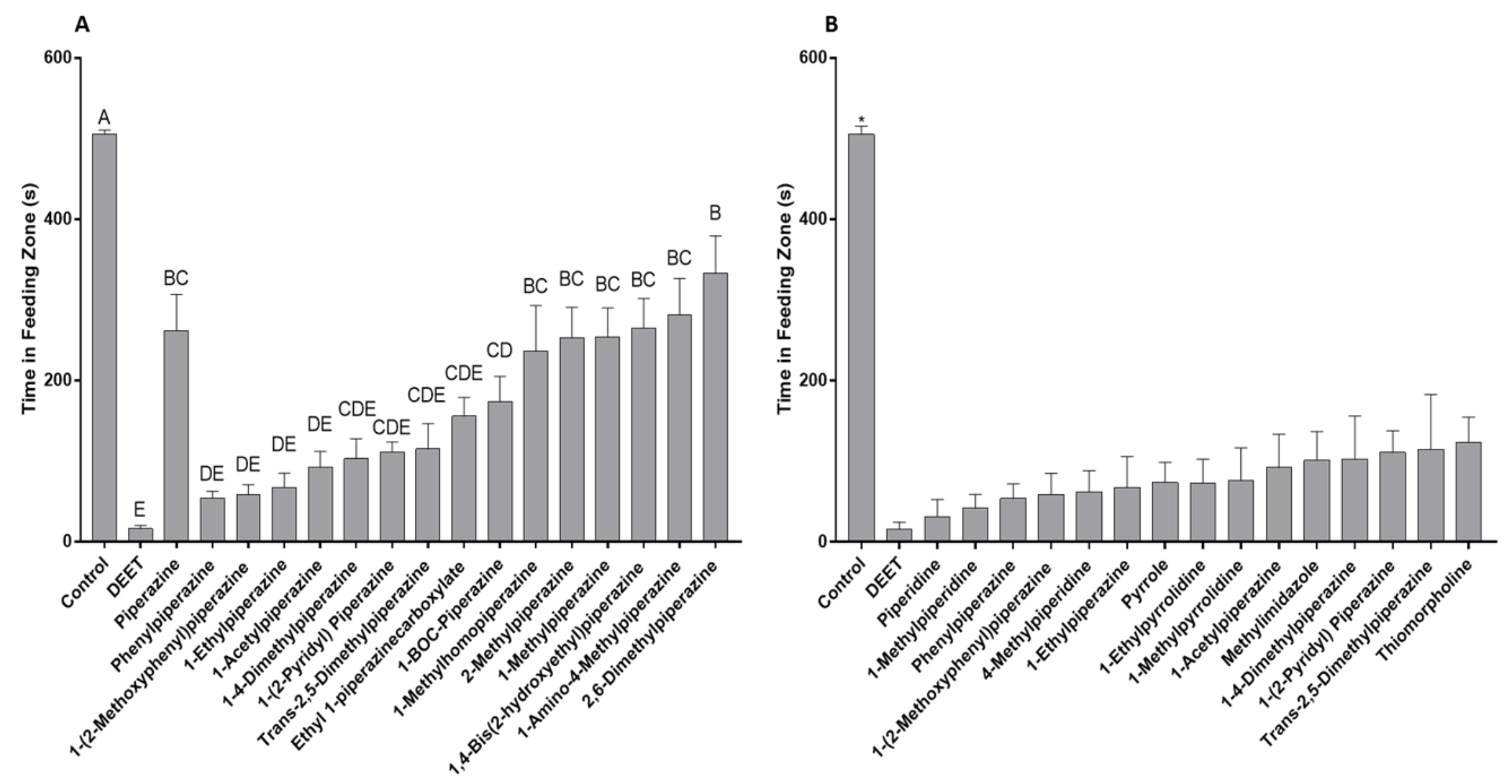
Figure 4. Effect of substituted piperazines on the feeding behavior of honey bees and compounds that are not significantly different from DEET results. Utilizing a video tracking protocol individual bees were screened in Petri dishes for behavior changes when exposed to sugar-agarose cubes treated with HCAs $(1 \% \mathrm{v} / \mathrm{v})$. Controls were sugar-agarose cubes with no treatment. DEET treated sugaragarose cubes were screened as a positive control. A) Substituted piperazines. B) Combined graph of HCAs that had deterrent effects similar to DEET. Vertical bars represent mean \pm SE. Different letters above bars indicate significant difference between means. One-way ANOVA with a Tukey's post hoc test $(\mathrm{P}<0.0001)$. 


\subsection{REFERENCES}

United States Environmental Protection Agency. 2017. https://www.epa.gov/insectrepellents/skin-applied-repellent-ingredients.

Atkins, E. L., R. L. Macdonald, and E. A. Greywood-Hale. 1975. Repellent additives to reduce pesticide hazards to honey bees : Field tests. Environ. Entomol. 4: 207-210.

Bernier, U. R., D. L. Kline, and K. H. Posey. 2007. Human emanations and related natural compounds that inhibit mosquito host-finding abilities, pp. 77-100. In Debboun, M., Frances, S.P., Strickman, D. (eds.), Insect Repellents Princ. Methods, Uses. CRC Press, Boca Raton.

Bernier, U. R., K. Posey, D. L. Kline, D. Barnard, and K. Chauhan; The United States of America as represented by the Secretary of Agriculture. 2012 Jun 26. Methods and compositions for repelling arthropods. U.S. patent 8,207,157B2

Brandt, A., A. Gorenflo, R. Siede, M. Meixner, and R. Büchler. 2016. The neonicotinoids thiacloprid, imidacloprid, and clothianidin affect the immunocompetence of honey bees (Apis mellifera L.). J. Insect Physiol. 86: 40-47.

Brown, M., and A. A. Hebert. 1997. Insect repellents: An overview. J. Am. Acad. Dermatol. 36: 243-249.

Callbreath, J. 1900. The use of carbolic acid during spraying time. Glean. Bee Cult. 841.

Carroll, J. F., J. P. Benante, J. A. Klun, C. E. White, M. Debboun, J. M. Pound, and W. Dheranetra. 2008. Twelve-hour duration testing of cream formulations of three repellents against Amblyomma americanum. Med. Vet. Entomol. 22: 144-151.

Chaimanee, V., J. D. Evans, Y. Chen, C. Jackson, and J. S. Pettis. 2016. Sperm viability and gene expression in honey bee queens (Apis mellifera) following exposure to the 
neonicotinoid insecticide imidacloprid and the organophosphate acaricide coumaphos. J. Insect Physiol. 89: 1-8.

Christen, V., F. Mittner, and K. Fent. 2016. Molecular effects of neonicotinoids in honey bees (Apis mellifera). Environ. Sci. Technol. 50: 4071-4081.

Cizelj, I., G. Glavan, J. Božič, I. Oven, V. Mrak, and M. Narat. 2016. Prochloraz and coumaphos induce different gene expression patterns in three developmental stages of the Carniolan honey bee (Apis mellifera carnica Pollmann). Pestic. Biochem. Physiol. 128: 68-75.

Collins, A. M., W. L. Rubink, J. I. Cuadriello Aguilar, and R. L. Hellmich II. 1996. Use of insect repellents for dispersing defending honey bees (Hymenoptera: Apidae). J. Econ. Entomol. 89: 608-613.

Das, S. K. 2016. Screening of bioactive compounds for development of new pesticides : a mini review. Univers. J. Agric. Res. 4: 15-20.

Dethier, V. G., B. L. Browne, and C. N. Smith. 1960. The designation of chemicals in terms of the responses they elicit from insects. J. Econ. Entomol. 53: 134-136.

Diaz, J. H. 2016. Chemical and plant-based insect repellents: efficacy, safety, and toxicity. Wilderness Environ. Med. 27: 153-163.

Dickens, J. C., and J. D. Bohbot. 2013. Mini review: mode of action of mosquito repellents. Pestic. Biochem. Physiol. 106: 149-155.

Dussaubat, C., A. Maisonnasse, D. Crauser, and S. Tchamitchian. 2016. Combined neonicotinoid pesticide and parasite stress alter honeybee queens' physiology and survival. Sci. Rep. 6: 314330.

Frances, S., D. Waterson, N. Beebe, and R. Cooper. 2004. Field evaluation of repellent 
formulations containing deet and picaridin against mosquitoes in Northern Territroy, Australia. J. Med. Entomol. 41: 414-417.

Free, J. B., J. A. Pickett, A. W. Ferguson, J. R. Simpkins, and M. C. Smith. 1985. Repelling foraging honeybees with alarm pheromones. J. Agric. Sci. 105: 255.

Garcia, M. D. G., S. U. Duque, A. B. L. Fernandez, A. Sosa, and A. R. FernandezAlba. 2017. Multiresidue method for trace pesticide analysis in honeybee wax comb by GC-QqQ-MS. Talanta. 163: 54-64.

Goulson, D. 2013. An overview of the environmental risks posed by neonicotinoid insecticides. J. Appl. Ecol. 50: 977-987.

Haarmann, T., M. Spivak, D. Weaver, B. Weaver, and T. Glenn. 2002. Effects of fluvalinate and coumaphos on queen honey bees (Hymenoptera: Apidae) in two commercial queen rearing operations. J. Econ. Entomol. 95: 28-35.

Hatjina, F., C. Papaefthimiou, L. Charistos, T. Dogaroglu, M. Bouga, C. Emmanouil, and G. Arnold. 2013. Sublethal doses of imidacloprid decreased size of hypopharyngeal glands and respiratory rhythm of honeybees in vivo. Apidologie. 44: $467-480$.

Johnson, R. M., L. Dahlgren, B. D. Siegfried, and M. D. Ellis. 2013. Acaricide, fungicide and drug interactions in honey bees (Apis mellifera). PLoS One. 8.

\section{Kakumanu, M. L., A. M. Reeves, T. D. Anderson, R. R. Rodrigues, M. A. Williams,} and M. A. Williams. 2016. Honey bee gut microbiome is sltered by in-hive pesticide exposures. Front. Microbiol. 7: 1-11.

Katz, T. M., J. H. Miller, and A. A. Hebert. 2008. Insect repellents: historical perspectives and new developments. J. Am. Acad. Dermatol. 58: 865-871. 
Klun, J. A., W. F. Schmidt, and M. Debboun. 2001. Stereochemical effects in an insect repellent. J. Med. Entomol. 38: 809-812.

Larson, N., and T. Anderson. 2017. Video tracking protocol to screen deterrent chemistries for honey bees. J. Vis. Exp. (In Press): e55603.

Lawrence, K. L., N. L. Achee, U. R. Bernier, K. D. Mundal, and J. P. Benante. 2014. Field evaluations of topical arthropod repellents in North, Central, and South America. J. Med. Entomol. 51: 980-988.

Moore, S. J., and M. Debboun. 2007. History of insect repellents, pp. 3-29. In Debboun, M., Frances, S.P., Strickman, D. (eds.), Insect Repellents Princ. Methods, Uses. CRC Press, Boca Raton.

Mullin, C. A., M. Frazier, J. L. Frazier, S. Ashcraft, R. Simonds, D. VanEngelsdorp, and J. S. Pettis. 2010. High levels of miticides and agrochemicals in North American apiaries: implications for honey bee health. PLoS One. 5: e9754.

Pettis, J. S., E. M. Lichtenberg, M. Andree, J. Stitzinger, R. Rose, and D. VanEngelsdorp. 2013. Crop pollination exposes honey bees to pesticides which alters their susceptibility to the gut pathogen Nosema ceranae. PLoS One. 8.

Sanchez-Bayo, F., and K. Goka. 2014. Pesticide residues and bees - A risk assessment. PLoS One. 9: 1-16.

Schreck, C. E., D. Fish, and T. P. McGovern. 1995. Activity of repellents applied to skin for protection against Amblyomma americanum and Ixodes scapularis ticks (Acari: Ixodidae). J. Am. Mosq. Control Assoc. 11: 136-40.

Solberg, V. B., T. A. Klein, K. R. McPherson, B. A. Bradford, J. R. Burge, and R. A. Wirtz. 1995. Field evaluation of deet and a piperidine repellent (A13- 37220) against 
Amblyomma americanum (Acari: Ixodidae). J. Med. Entomol. 32: 870-875.

Taylor, K. S., G. D. Waller, and L. A. Crowder. 1987. Impairment of a classical conditioned response of the honey bee (Apis mellifera L.) by sublethal doses of synthetic pyrethroid insecticides. Apidologie. 18: 243-252.

Teeters, B. S., R. M. Johnson, M. D. Ellis, and B. D. Siegfried. 2012. Using videotracking to assess sublethal effects of pesticides on honey bees (Apis mellifera L.). Environ. Toxicol. Chem. 31: 1349-1354.

Tison, L., M. L. Hahn, S. Holtz, A. Robner, U. Greggers, G. Bischoff, and R. Menzel. 2016. Honey bees' behavior is impaired by chronic exposure to the neonicotinoid thiacloprid in the field. Environ. Sci. Technol. 50: 7218-7227.

vanEngelsdorp, D., and M. D. Meixner. 2010. A historical review of managed honey bee populations in Europe and the United States and the factors that may affect them. J. Invertebr. Pathol. 103: S80-S95.

Warnhoff, E. W. 1998. When piperidine was a structural problem. Bull. Hist. Chem. 22: 29-34.

Williams, J. R. 2016. Biomarkers of oxidative stress in atrazine-treated honey bees: A laboratory and in-hive study. M.S. thesis, Virginia Polytechnic and State University, Virginia.

Williamson, S. M., and G. A. Wright. 2013. Exposure to multiple cholinergic pesticides impairs olfactory learning and memory in honeybees. J. Exp. Biol. 216: 1799-1807. 


\section{CHAPTER 6: EFFECT OF PIPERIDINE ON THE FORAGING BEHAVIOR OF HONEY BEES}

\subsection{ABSTRACT}

Protecting honey bees (Apis mellifera L.) from exposure to pesticide treated crops is a high priority for beekeepers. Pesticides can have several detrimental effects on honey bees and their colonies including reducing their ability to deal with infections, increasing the toxicity of other pesticides, and altering social behaviors. Utilizing a repellent compound to keep bees away from a pesticide treated crop is a method that could potentially be employed to reduce interaction times between foraging bees and these treated crops. A previous study found that several heterocyclic amines (HCA) elicited repellent behaviors in bees when they were exposed to these compounds within food cubes in a laboratory experiment. The aim of this study was to determine if the repellent effect was conserved when taken into semi-field and field assays. The most efficacious HCA from the previous study was piperidine. This compound was subsequently chosen to be screened in a semi-field high tunnel assay and two field studies to determine if repellency was still achieved. Nucleus honey bee colonies were transferred into high-tunnels and the bees from these colonies were trained to a sugar feeder in the opposite corner. Video recording devices were used to monitor visitation and recruitment to the feeder over an hour period. A feeder that had been treated with $1 \%$ piperidine $(\mathrm{v} / \mathrm{v})$ significantly reduced visitation to the feeder by $97 \%$ over a 60 min period compared to a control feeder. Piperidine solutions were also sprayed onto melon flowers and knapweed bundles to determine if repellency was conserved in field studies. Melon flowers sprayed with control sprays had an average of $6 \pm 1.4$ bee visitors during the observational period. Spraying the flowers with a $1 \%$ piperidine $(\mathrm{v} / \mathrm{v})$ 
solution reduced this number by half to $3 \pm 1.2$ visitors. Knapweed showed a similar trend going from $8 \pm 1.5$ foragers to $1.5 \pm 0.4$ foragers. The standard repellent DEET was shown to elicit similar results when sprayed on the melon and knapweed flowers.

\subsection{INTRODUCTION}

The development of novel protection methods and policies is paramount for the continued success of honey production and pollination services that are worth billions of dollars worldwide (Gallai et al. 2009, Mullin et al. 2010). Globally, there has been an increase in managed honey bee colony numbers; however, a couple of regions have seen significant declines and yearly losses that are a cause for concern (Aizen and Harder 2009, vanEngelsdorp and Meixner 2010, Seitz et al. 2016). The largest declines were reported in the United States and Europe, where millions of managed bee colonies were lost over the course of 60 years (vanEngelsdorp and Meixner 2010). The apiculture industry has since struggled to increase the number of managed colonies due to annual losses. This is especially apparent in the United States where yearly losses have been estimate to be around $49 \%$ for beekeepers (Seitz et al. 2016).

There has been contention as to what is the cause behind these losses, but it is now thought that a number of interacting stressors that affect colony survival rates (Potts et al. 2010). The types of stressors that bee colonies face range from genetic variation to climate conditions (vanEngelsdorp and Meixner 2010). There are also pests, pathogens, and pesticide use that have detrimental effects on bee colonies (Aizen and Harder 2009, vanEngelsdorp and Meixner 2010, Smith et al. 2013, Lee et al. 2015). Direct poisoning of

bee colonies through field applications of pesticides has decreased due to policies such as 
the European Council Directive 91/414 and the United States Federal Insecticide Fungicide and Rodenticide Act (vanEngelsdorp and Meixner 2010). However, these policies are still dependent on users following pesticide application guidelines, and despite the recommendations, pesticides can still be found in bee colonies and can cause acute and sublethal effects (Mullin et al. 2010, vanEngelsdorp and Meixner 2010). Previous studies report pesticides to weaken the immune system and alter social and foraging behaviors of bees (Brandt et al. 2016, Christen et al. 2016, Tison et al. 2016). Additionally, there has been an increase in agriculture for pollinator dependent crops, which in turn can strain available bee colonies used for largescale pollination services (Aizen and Harder 2009). Thus, crops will likely need to be sprayed with pesticides to ensure high yields, which can increase the chance that bees will come into contact with these pesticides (vanEngelsdorp and Meixner 2010). One method to prevent this incidental exposure to the pesticides would be to use a repellent to keep the foraging bees from the crop until pesticide levels diminish.

DEET ( $N, N$-diethyl-m-toluamide) is currently considered to be the gold standard in arthropod repellent testing, and as such, is recommended by the World Health Organization and the United States Environmental Protection Agency (EPA) for use as a positive control in novel repellent testing (Katz et al. 2008, Dickens and Bohbot 2013, Lawrence et al. 2014). However, DEET's safety has repeatedly been questioned over the years. This has led to research into developing alternatives that are proven safe for human use (Diaz 2016). Included in this push for new repellent compounds is the re-examination of piperidine-like compounds discovered in the 70's (Moore and Debboun 2007). 
Piperidine, a compound discovered in 1852 from black pepper, is a heterocyclic amine (HCA) that has a ring structure consisting of five carbons and one nitrogen (Warnhoff 1998). Several analogs of piperidine have been shown to be affective in repelling a number of different arthropods including chigger mites, ticks, and mosquitoes (Schreck et al. 1995, Solberg et al. 1995, Klun et al. 2001), this includes Picaridin which is registered with the EPA as an active ingredient for tick repellents (Carroll et al. 2008, "United States Environmental Protection Agency" 2017). The proposed mode of action, of the HCAs, is through induction of hyposmia or anosmia ultimately making it impossible for the target to find the intended host (Bernier et al. 2012). It has since been found that HCA treated sugar-agarose cubes effectively deter bees from the food source (Chapter 5). Based on the above findings, I hypothesized that treating a sugar feeder and spraying crops with the most efficacious HCA, determined by the prior study (Chapter 5), would repel bees from each food source. In this study, I treated a 50\% (w/v) sugar solution feeder with piperidine (final concentration $1 \% \mathrm{v} / \mathrm{v}$ ), and additionally sprayed plots of melon flowers (Cucumis melo L.) and bundles of knapweed (Centaurea stoebe L.) with a $1 \%$ (v/v) piperidine solution. The sugar feeder was monitored over a 2-hr period with video recording equipment, while filed observations were conducted for the melon flowers and knapweed bundles. It was found that treatment with $1 \%(\mathrm{v} / \mathrm{v})$ piperidine significantly reduced forager visitation in all scenarios.

\subsection{MATERIALS AND METHODS}

\subsubsection{Chemicals}

Piperidine was purchased from Sigma-Aldrich (St. Louis, MO). Deep Woodsman OFF was used as the source for DEET (98\% active ingredient; $N, N$-Diethyl-meta-toluamide). 


\subsubsection{High-tunnel experiments}

A high-tunnel protocol described by Wilson (2016) was used to evaluate the number of bees visiting a feeder station treated with and without the HCA piperidine (Fig. 1). Two nucleus bee colonies were established by splitting larger colonies from the Kentland Farms of Virginia Tech (Blacksburg, VA). These nucleus colonies were moved to the Price's Fork Research Center of Virginia Tech where each colony was placed into a separate high tunnel. The high tunnel dimensions are $10.97 \mathrm{~m} \mathrm{~L} \mathrm{x} 7.92 \mathrm{~m} \mathrm{~W}$ x $3.66 \mathrm{~m} \mathrm{H}$. The bees from each nucleus colony were trained to a feeding station containing $50 \%(\mathrm{w} / \mathrm{v})$ sucrose solution for $2 \mathrm{~d}$. The feeding stations were placed in front of the hive in the morning for a 2-h period. After the 2-h period, the sucrose solution was replaced with water only and left in the same location overnight. The next morning, the water was replaced with the sucrose solution and the feeding stations were moved $10 \mathrm{~m}$ from the hive. Once the bees were trained to the feeding stations, one of the feeding stations was supplemented with $1 \%(\mathrm{v} / \mathrm{v})$ piperidine (HCA treatment), while the other feeding station contained the sucrose solution only (control treatment). A video camera was used to record the number of bees visiting the control and piperidine-treated feeding stations for a 2-h period. The video cameras were programmed to take pictures of the visiting bees at 1-min intervals. The video recordings were analyzed at 5-min intervals for 60 min using ImageJ software (National Institutes of Health). Each experiment was repeated in duplicate with two colonies per treatment. Linear regression analysis was used for each treatment with GraphPad Prism software (La Jolla, CA). 


\subsubsection{Field experiments with melon flowers and knapweed}

A nucleus bee colony was moved from the Moore Farm of Virginia Tech to melon plots at Virginia Tech's Kentland Farm. The melons were in 5 plots with 4 rows per plot and $5 \mathrm{ft}$ in between each plot. Three observers were assigned to each plot and each observer was assigned one row in the plot. The observers were randomly assigned a 1-L spray bottle containing $250 \mathrm{ml}$ deionized $\mathrm{H}_{2} \mathrm{O}$ (control treatment), 1\% (v/v) DEET (repellent treatment), or $1 \%(\mathrm{v} / \mathrm{v})$ piperidine (HCA treatment). Each observer positioned themselves near 20 melon flowers and recorded the number of bees visiting the untreated flowers for a 3-min period. A bee visit was recorded as one individual landing and actively foraging on the flower. Those bees that landed on and immediately left the flower were not reported as a visitation. Melon flower pollen is less desirable to honey bees than other pollen so in addition to honey bees, the number of squash bees, Peponapis pruinosa Say, visiting the flowers was recorded by each observer. After the initial 3-min observation period, an observer delivered the treatments to each of the flowers with a single pump of the spray bottle assigned to the observer. A second 3-min observation period was conducted and the number of bees visiting the flowers was recorded following the application of treatments. The spray bottles containing the treatments were randomly assigned again, and same method was applied to the next melon plot. The experiment was performed over $2 \mathrm{~d}$ with a total of 12, 3-min pre- and post-spray observations. The pre- and post-number of bees visiting the treated melon flowers was statistically analyzed using an ANCOVA with JMP (SAS, Cary, NC). The pre-treatment bee visits were used as the covariate and the ANCOVA was valid if the interaction between the treatment and covariate was non- 
significant $(P<0.05)$. A student's $t$-test was used to determine the significance between the post-visitation of bees to the treatments.

A section of knapweed, located at the Prices Fork Research Center apiary, was divided into $301 \times 1 \mathrm{~m}$ bundles. There was $c a .1 \mathrm{~m}$ between each bundle. Three observers were each assigned to a bundle. The observers were randomly assigned a 1-L spray bottle containing $250 \mathrm{ml}$ deionized $\mathrm{H}_{2} \mathrm{O}$ (control treatment), $1 \%$ (v/v) DEET (repellent treatment), or 1\% $(\mathrm{v} / \mathrm{v})$ piperidine (HCA treatment). Each observer positioned themselves near their assigned bundle and recorded the number of bees visiting the untreated bundle for a 3-min period. A bee visit was recorded as one individual landing and actively foraging on the flower. Those bees that landed on and immediately left the flower were not reported as a visitation. After the initial 3-min observation period, an observer delivered the treatment to the bundle, spraying to saturation ( $c a$. 20-25 pumps), with the spray bottle assigned to the observer. A second 3-min observation period was conducted and the number of bees visiting the bundles was recorded following the application of treatments. The spray bottles containing the treatments were randomly assigned again, and same method was applied to the next set of knapweed bundles. The experiment was performed over 3 days with a total of 17, 3 min pre- and post-spray observations. The pre- and post-number of bees visiting the treated knapweed bundles was statistically analyzed using an ANCOVA with JMP (SAS, Cary, NC). The pre-treatment bee visits were used as the covariate and the ANCOVA was valid if the interaction between the treatment and covariate was nonsignificant $(P<0.05)$. A student's $t$-test was used to determine the significance between the post-visitation of bees to the treatments. 


\subsection{RESULTS}

\subsubsection{High-tunnel experiments}

The high-tunnel experiments were conducted to examine the effect of piperidine on the number of bees visiting a feeding station a sucrose-based food source. The maximum number of bees visiting the control (sucrose solution only) feeding station was 112 individuals in a $60-$ min period. The maximum number of bees visiting the treated (1\% piperidine in sucrose solution) feeding station was 9 individuals in a 60 -min period. The average number of bees visiting a control feeder over the $60 \mathrm{~min}$ period was $91 \pm 2.6$, compared to the treated feeder with an average of $3 \pm 0.4$. A steady recruitment of bees was observed visiting the control feeding station $\left(\mathrm{y}=0.6576 \mathrm{x}+0.0952, R^{2}=0.83\right)$ whereas a fewer number of bees visited the treatment feeding station $\left(\mathrm{y}=0.0103+0.0175, R^{2}=\right.$ 0.03) (Fig. 2).

\subsubsection{Field experiments with melon flowers and knapweed}

The semi-field experiments were conducted to determine if DEET and piperidine can deter honey and squash bees from visiting melon flowers and honey bees from visiting knapweed. There was no significant effect between the pre-treatment and post-treatment number of bees visiting the melon flowers $(P=0.46)$. However, there was a significant reduction in the number of bees visiting the melon flowers treated with DEET and piperidine compared to those bees visiting the untreated melon flowers (Fig. 3). The number of bees visiting the melon flowers treated with DEET and piperidine was $1.6 \pm$ 0.76 and $3 \pm 1.2$ bees per group of 20 treated flowers, respectively, compared to the $6.1 \pm$ 
1.4 bees per group of 20 untreated flowers. There was no significant difference between the number of bees visiting the DEET-treated melon flowers compared the number of bees visiting the melon flowers treated with piperidine.

There was no significant effect between the pre-treatment and post-treatment number of bees visiting the knapweed $(P=0.95)$. However, there was a significant reduction in the number of bees visiting the knapweed treated with DEET and piperidine compared to those bees visiting the untreated knapweed (Fig. 3). The number of bees visiting the knapweed treated with DEET and piperidine was $1.1 \pm 0.71$ and $1.5 \pm 0.41$ bees per treated bundle, respectively, compared to the $8.2 \pm 1.5$ bees per untreated bundle (Fig. 3). There was no significant difference between the number of bees visiting the DEET-treated knapweed compared the number of bees visiting the knapweed treated with piperidine.

\subsection{DISCUSSION}

This study provides evidence that DEET and piperidine can deter foraging bees away from

food sources. It was observed that forager bee visitation and recruitment were significantly reduced to food sources that were treated with these compounds. Piperidine significantly reduced the visitation and recruitment of foraging bees trained to a feeder station when added to the sugar solution. These findings are supported by observations from previous experiments where individual bees spent less time on food cubes, in a lab, treated with piperidine compared the food cubes without piperidine treatment (Chapter 5). The effects of DEET on forager bee visitation and recruitment to the feeder station was not examined due to the unavailability of nucleus bee colonies. 
Piperidine is a six-membered ring structure, consisting of 5 carbons and one nitrogen, that was isolated from black pepper in 1852 (Warnhoff 1998). Piperidine analogs have been reported to repel several types of arthropods. The analog 1-(3-cyclohexen-1-ylcarbonyl)2-methylpiperidine was not only reported to repel the lone star tick, Amblyomma americanum, away from humans significantly longer than DEET, but a stereoisomer of the analog was reported to be an effective repellent for mosquitoes and chigger mites (Solberg et al. 1995, Klun et al. 2001). Schreck et al. (1995) reported the analog 1-(3-cyclohexenylcarbonyl) piperidine to provide protection against $A$. americanum 1.5 times longer than DEET. The Environmental Protection Agency currently recognizes 7 active ingredients as topical insect repellents, including Picaridin, which is a highly effective piperidine-based tick repellent (Carroll et al. 2008, “United States Environmental Protection Agency” 2017). The hypothesis of piperidine-based compounds will deter bees from a food source was based on the success of these compounds to repel other arthropods. In fact, this study demonstrates that piperidine does significant deter bees from melon flowers and knapweed by reducing the visitation of foraging individuals to the flowers. A similar effect was observed with the number of bees visiting melon flowers and knapweed treated with DEET.

DEET is a gold standard for arthropod repellency testing and has previously been shown to repel bees away from a "threat" to their hive (Collins et al. 1996, Lawrence et al. 2014). DEET has been shown to be a highly effective gustatory repellent to bees when infused into a sugar solution (Atkins et al. 1975 A). It was observed in this study that foraging 
bees would approach the DEET- and piperidine-treated melon flowers and knapweed, but would leave before contacting the melon flowers and knapweed, which may be related to an olfactory response to the compounds. Bernier et al. (2005) and Abramson et al. (2010) have observed DEET to not be a suitable olfactory repellent, but rather a contact repellent. There are several reports of piperidine analogs to be contact repellents (Schreck et al. 1995, Solberg et al. 1995, Klun et al. 2001). In addition, DEET has been found to be phytotoxic to alfalfa and cotton foliage (Atkins, Macdonald, et al. 1975) and, thus, might result in the plants emitting other volatiles that repel the bees.

Overall, this study provides evidence that the heterocyclic amine piperidine deters bees from a treated crop and this deterrent effect is comparable to that of DEET. While the mode of detection is not fully understood for the bees, there is a need for further investigation into the sensory physiology of bees exposed to piperidine and DEET. These data suggest possible use of chemical interventions, such as piperidine, to reduce the contact of foraging bees with a pesticide-treated food source. Although, a further examination of piperidine-based compound stability and efficacy is warranted for the continued development of these chemistries as pollinator protectants for the agricultural and apicultural industry. 


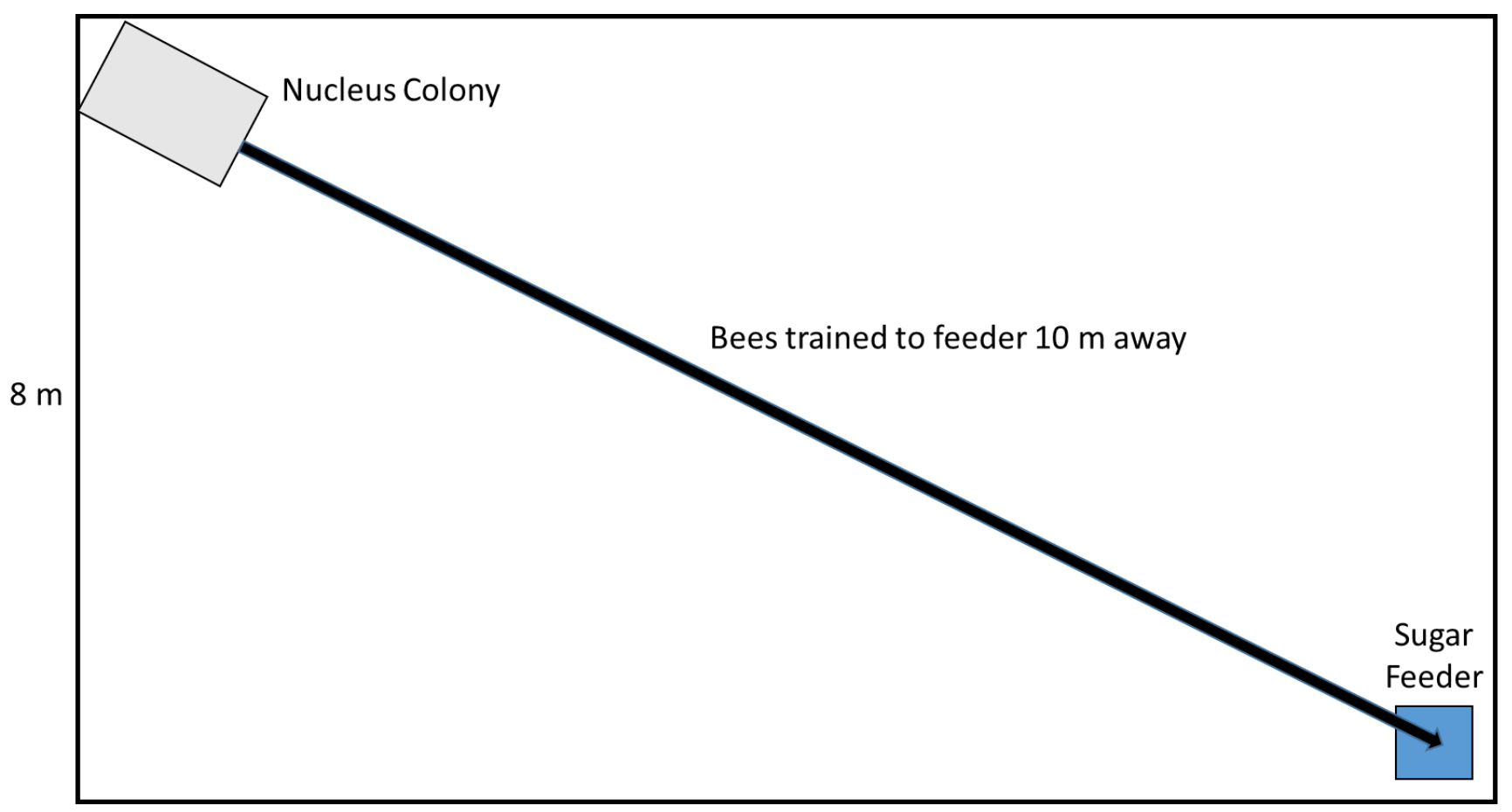

$23 \mathrm{~m}$

Figure 1. Diagram of semi-field experiments. Two nucleus colonies were placed into the corner of identical high-tunnels and the bees were trained to a sugar feeders $(50 \% \mathrm{w} / \mathrm{v})$ located $10 \mathrm{~m}$ away in the opposite corners of the tunnels for 9 days. On the $10^{\text {th }}$ day the one of the sugar feeders had an additional $1 \%(\mathrm{v} / \mathrm{v})$ piperidine added to it. Visitation to the feeders was then recorded using GoPro ${ }^{\circledR}$ cameras for $1 \mathrm{hr}$. 


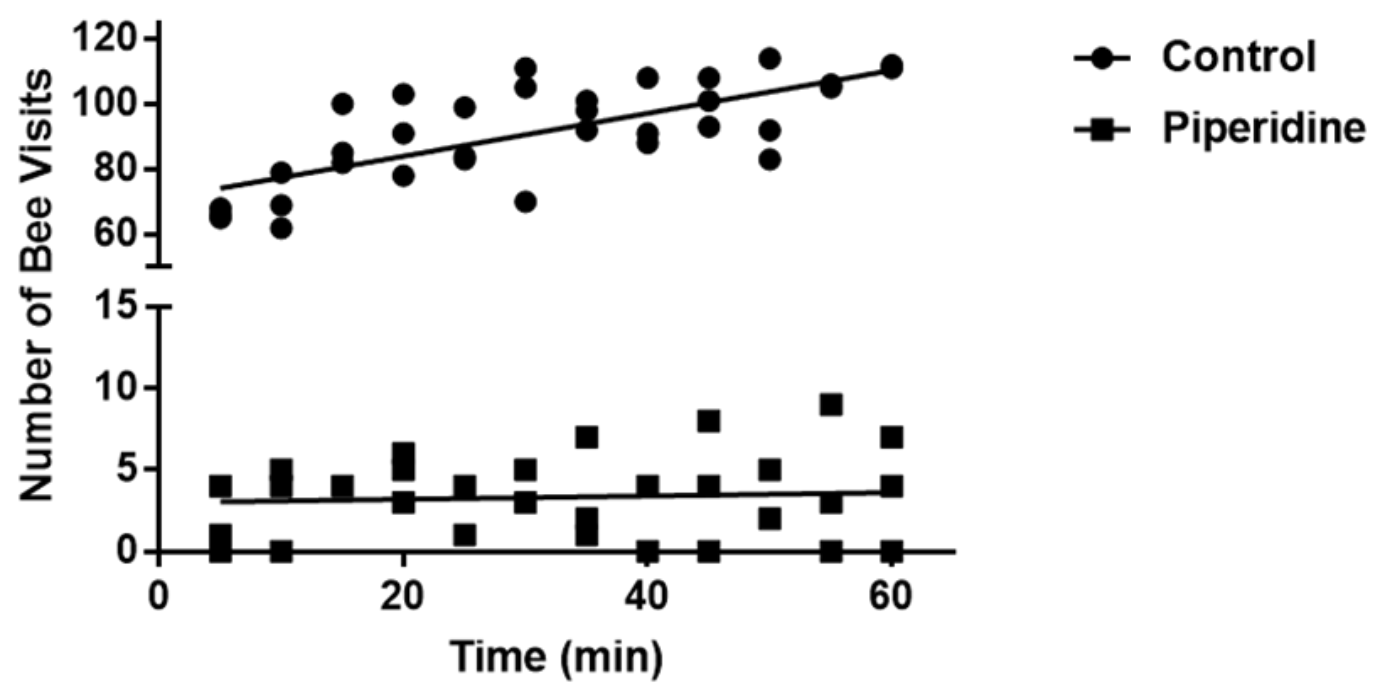

Figure 2. Number of honey bees visiting a feeder station treated with piperidine. Two high tunnels contained two nucleus bee colonies that were trained to a feeder station containing $50 \%$ (w/v) sucrose solution for $9 \mathrm{~d}$. The sucrose solution was replaced at the feeder station with $1 \%$ (v/v) piperidine a sucrose solution on day 10. The number of bees visiting the piperidine-untreated (control) and -treated feeder stations was video recorded for $60 \mathrm{~min}$. Data were analyzed using linear regression analysis. Control equation: $\mathrm{Y}=0.6578 * \mathrm{X}+70.85, \mathrm{R}^{2}=0.53$. Treatment equation: $\mathrm{Y}=0.01026 * \mathrm{X}+3, \mathrm{R}^{2}=0.005$. 

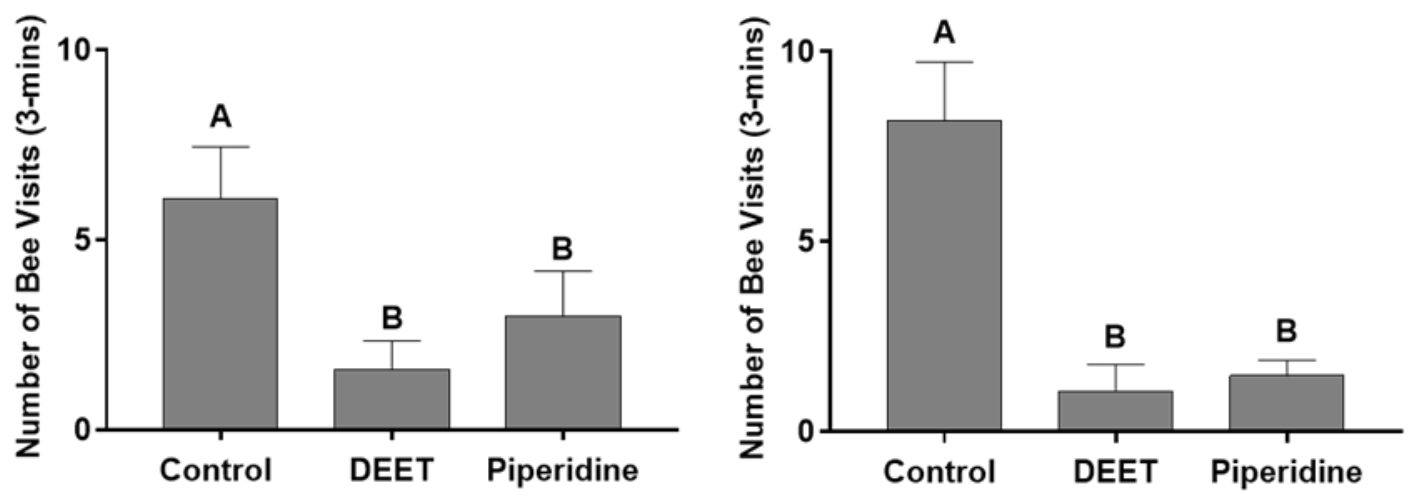

Figure 3. Number of pollinators visiting DEET- and piperidine-treated melon flowers (A) and knapweed bundles $(\mathbf{B})$. Melon flowers $(N=240)$ and knapweed bundles $(N=51)$ were treated with deionized $\mathrm{H}_{2} \mathrm{O}$ (control), $1 \%(\mathrm{v} / \mathrm{v})$ DEET, or $1 \%(\mathrm{v} / \mathrm{v})$ piperidine. A 3-min postspray count of visitation was performed and repeated 12 times in the melon plots and 17 times in the field of knapweed. A) The number of honey bees and squash bees visiting the melon flowers post-spray $(\mathrm{F}=2,35 ; \mathrm{F}$ ratio $=7.6 ; P=0.002)$. B $)$ The number of honey bees visiting knapweed bundles post spray $(\mathrm{F}=2,45 ; \mathrm{F}$ ratio $=15.4 ; P<0.001)$. Vertical bars represent the mean \pm standard error. Different letters above the bars indicate a significant difference between the treatment means. Data were analyzed using an ANCOVA with a student's multiple comparison t-test. 


\subsection{REFERENCES}

(United States Environmental Protection Agency) . 2017. United States Environmental Protection Agency. (https://www.epa.gov/insect-repellents/skin-applied-repellentingredients).

Aizen, M. A., and L. D. Harder. 2009. The global stock of domesticated honey bees is growing slower than agricultural demand for pollination. Curr. Biol. 19: 915-918.

Atkins, E. L., R. L. Macdonald, and E. A. Greywood-Hale. 1975. Repellent additives to reduce pesticide hazards to honey bees : Field tests. Environ. Entomol. 4: 207-210.

Atkins, W., R. L. MacDonald, T. P. McGovern, M. Beroza, and E. A. Greywood-Hale. 1975. Repellent additives to reduce pesticide hazards to honeybees: Laboratory testing. J. Apic. Res. 14: 85-97.

Bernier, U. R., K. Posey, D. L. Kline, D. Barnard, and K. Chauhan; The United States of America as represented by the Secretary of Agriculture. 2012 Jun 26. Methods and compositions for repelling arthropods Methods and compositions for repelling arthropods. U.S. patent 8,207,157B2.

Brandt, A., A. Gorenflo, R. Siede, M. Meixner, and R. Büchler. 2016. The neonicotinoids thiacloprid, imidacloprid, and clothianidin affect the immunocompetence of honey bees (Apis mellifera L.). J. Insect Physiol. 86: 40-47.

Carroll, J. F., J. P. Benante, J. A. Klun, C. E. White, M. Debboun, J. M. Pound, and W. Dheranetra. 2008. Twelve-hour duration testing of cream formulations of three repellents against Amblyomma americanum. Med. Vet. Entomol. 22: 144-151.

Christen, V., F. Mittner, and K. Fent. 2016. Molecular effects of neonicotinoids in honey bees (Apis mellifera). Environ. Sci. Technol. 50: 4071-4081. 
Collins, A. M., W. L. Rubink, J. I. Cuadriello Aguilar, and R. L. Hellmich II. 1996. Use of insect repellents for dispersing defending honey bees (Hymenoptera: Apidae). J. Econ. Entomol. 89: 608-613.

Diaz, J. H. 2016. Chemical and plant-based insect repellents: efficacy, safety, and toxicity. Wilderness Environ. Med. 27: 153-163.

Dickens, J. C., and J. D. Bohbot. 2013. Mini review: mode of action of mosquito repellents. Pestic. Biochem. Physiol. 106: 149-155.

Gallai, N., J. M. Salles, J. Settele, and B. E. Vaissière. 2009. Economic valuation of the vulnerability of world agriculture confronted with pollinator decline. Ecol. Econ. 68: $810-821$.

Katz, T. M., J. H. Miller, and A. A. Hebert. 2008. Insect repellents: historical perspectives and new developments. J. Am. Acad. Dermatol. 58: 865-871.

Klun, J. A., W. F. Schmidt, and M. Debboun. 2001. Stereochemical effects in an insect repellent. J. Med. Entomol. 38: 809-812.

Lawrence, K. L., N. L. Achee, U. R. Bernier, K. D. Mundal, and J. P. Benante. 2014. Field evaluations of topical arthropod repellents in North, Central, and South America. J. Med. Entomol. 51: 980-988.

Moore, S. J., and M. Debboun. 2007. History of insect repellents, pp. 3-29. In Debboun, M., Frances, S.P., Strickman, D. (eds.), Insect Repellents Princ. Methods, Uses. CRC Press, Boca Raton.

Mullin, C. A., M. Frazier, J. L. Frazier, S. Ashcraft, R. Simonds, D. VanEngelsdorp, and J. S. Pettis. 2010. High levels of miticides and agrochemicals in North American apiaries: implications for honey bee health. PLoS One. 5: e9754. 
Potts, S. G., J. C. Biesmeijer, C. Kremen, P. Neumann, O. Schweiger, and W. E. Kunin. 2010a. Global pollinator declines: Trends, impacts and drivers. Trends Ecol. Evol. 25: 345-353.

Potts, S. G., J. C. Biesmeijer, C. Kremen, P. Neumann, O. Schweiger, and W. E. Kunin. 2010b. Global pollinator declines: Trends, impacts and drivers. Trends Ecol. Evol. 25: 345-353.

Schreck, C. E., D. Fish, and T. P. McGovern. 1995. Activity of repellents applied to skin for protection against Amblyomma americanum and Ixodes scapularis ticks (Acari: Ixodidae). J. Am. Mosq. Control Assoc. 11: 136-40.

Seitz, N., K. S. Traynor, N. Steinhauer, K. Rennich, M. E. Wilson, J. D. Ellis, R. Rose, D. R. Tarpy, R. R. Sagili, D. M. Caron, K. S. Delaplane, J. Rangel, K. Lee, K. Baylis, J. T. Wilkes, J. A. Skinner, J. Pettis, and D. VanEngelsdorp. 2016. A national survey of managed honey bee 2014-2015 annual colony losses in the USA. J. Apic. Res. 54: 292-304.

Smith, K. M., E. H. Loh, M. K. Rostal, C. M. Zambrana-Torrelio, L. Mendiola, and P. Daszak. 2013. Pathogens, pests, and economics: Drivers of honey bee colony declines and losses. Ecohealth. 10: 434-445.

Solberg, V. B., T. A. Klein, K. R. McPherson, B. A. Bradford, J. R. Burge, and R. A. Wirtz. 1995. Field evaluation of Deet and a piperidine repellent (A13- 37220) against Amblyomma americanum (Acari: Ixodidae). J. Med. Entomol. 32: 870-875.

Tison, L., M. L. Hahn, S. Holtz, A. Robner, U. Greggers, G. Bischoff, and R. Menzel. 2016. Honey bees' behavior is impaired by chronic exposure to the neonicotinoid thiacloprid in the field. Environ. Sci. Technol. 50: 7218-7227. 
vanEngelsdorp, D., and M. D. Meixner. 2010. A historical review of managed honey bee populations in Europe and the United States and the factors that may affect them. J. Invertebr. Pathol. 103: S80-S95.

Warnhoff, E. W. 1998. When piperidine was a structural problem. Bull. Hist. Chem. 22: $29-34$.

Wilson, J. M. 2016. The effects of insecticides on squash bugs, its' egg parasitoid and pollinators in Virginia cucurbit production. Ph.D. dissertation, Virginia Polytechnic and State University, Virginia. 


\section{CHAPTER 7: ELECTROANTENNOGRAM RESPONSES OF HONEY BEE ANTENNAE TO PHEROMONAL AND HETEROCYCLIC AMINE COMPOUNDS}

\subsection{ABSTRACT}

Agricultural practices require the use of pesticides to ensure that crop yields remain high to support human need. However, pesticide applications can have adverse effects on the pollinators that visit the crops. Honey bee foragers can bring pesticide residues back to their colonies which then can spread to the other inhabitants resulting in decreased health of the colony. To better protect bee foragers from pesticide exposure a repellent could be utilized to keep them away from a treated area for a short amount of time. Previous studies have shown that honey bee pheromonal compounds and several heterocyclic amines (HCA) elicit repellent effects in laboratory tests and field experiments. The proposed method of action for both groups of compounds is through olfactory activation within bee antennae. The aim of this study was to verify the method of detection by bees. This was done by using electroantennogram recordings. Bee antennae were excised and suspended between two electrodes. Pheromonal compounds and HCAs were passed over the antennae and the voltage changes were recorded. It was found that all the pheromonal compounds elicited some response in the antennae, however only synthetic Nasonov pheromone, citral, and geraniol elicited responses that were significantly different from control and vehicle detections. The HCAs were found to have no significant effect on bee antennae at the concentrations (1\% v/v) used in the previous studies. The HCAs piperidine and pyrrolidine were not significantly different from control and vehicle responses until a 10-fold increase in concentration was used. Piperidine elicited the strongest responses in antennae 
averaging $22 \pm 2.6 \mathrm{mV}$ when $100 \%$ compound was used. The repellent standard DEET was found to have no significant effect on bee antennae at $100 \%$ concentration.

\subsection{INTRODUCTION}

The honey bee, Apis mellifera L., is an agriculturally and economically important arthropod (Gallai et al. 2009). Pesticide exposure can have deleterious effects on honey bee colonies (Mullin et al. 2010, vanEngelsdorp and Meixner 2010). With need for continued use of these chemicals to provide sufficient crop yields in our agriculture novel strategies for pollinator protection are required (Mullin et al. 2010). One approach to decreasing the exposure time of pollinators with pesticides is to use repellent chemistries to deter foraging bees from pesticide treated crops.

Historically, insect repellents (IRs) have been used to prevent the spread of deadly diseases by arthropod vectors (Katz et al. 2008), however since 1900, studies have suggested repellent before or with a pesticide spray could benefit pollinators (Callbreath 1900, Johansen 1960, Woodrow et al. 1965, Free et al. 1985, Solomon and Hooker 1989). Synthetic and naturally occurring compounds have been shown to elicit repellent effects in honey bees (Woodrow et al. 1965). Honey bee Nasonov pheromone components have been found to have repellent and deterrent effects in a snail and parasitic mite (Hoppe and Ritter 1988, Price and Berry 2008).

The synthetic repellent DEET is recognized by the WHO and EPA as the standard in arthropod repellency (Moore and Debboun 2007, Lawrence et al. 2014) and has been 
shown to disperse bees from marked threats to hives (Collins et al. 1996). Additionally, the EPA currently recognizes 7 active ingredients for topically applied insect repellents including Picaridin, a piperidine compound shown to be highly effective tick repellent (Carroll et al. 2008, "United States Environmental Protection Agency" 2017). Piperidine is a six-membered ring consisting of 5 carbon and one nitrogen group. It was isolated from black pepper in 1852 (Warnhoff 1998). Analogs of this heterocyclic amines (HCA) have been found to repel several types of arthropods. 1-(3-cyclohexen-1-ylcarbonyl)-2methylpiperidine was shown to repel the lone star tick Amblyomma americanum L. away from humans significantly longer than DEET and one of its stereoisomers has been shown to be effective in repelling mosquitoes and chiggers (Solberg et al. 1995, Klun et al. 2001). A patent was filed in 2012 for a novel method of repelling mosquitoes using HCAs by causing hyposmia and anosmia within mosquitoes (Bernier et al. 2012). In a prior study, honey bees were deterred from food sources using honey bee pheromones and 31 heterocyclic amines (HCAs) (Chapter 5). The pheromonal compounds, and 16 of the HCAs, were as effective as the standard repellent DEET in deterring individual honey bees from food cubes (Chapter 4).

The first EAG method was developed by Schneider (1957) following the observation of voltage fluctuations between the base and tip of a Bombyx mori L. antenna exposed to intraspecific pheromones. The exact mechanism for these fluctuations is not known; however, Schneider (1963) postulated that the electroantennogram responses were generated by many odorant receptor (OR) potentials being summed in response to a stimulus (Schneider 1963, Syntech 2015). ORs are found on odorant receptor neurons 
(ORNs) housed within sensilla located on the antennae. Odorants enter the sensilla through pores and then are transported by odorant binding proteins (OBPs) to the ORs. The OBP/odorant complex then activates the OR sending a signal down the ORN to the insect brain processing centers where a response to the odorant is generated (Hildebrand and Shepherd 1997, Leal 2013, Riffel and Hildebrand 2016). In an EAG, an odorant is puffed over an antennae connected to electrodes and the resulting voltage fluctuations are amplified so that they can be registered by a recording device (Syntech 2015). The EAG method was initially used for the identification of pheromones; however, it was quickly coupled with gas chromatography (GC) (James et al. 1952, Schneider 1957, Roelofs and Comeau 1971, Arn et al. 1975). This combination has expanded the applications of the EAG into host-odor volatile identification thereby allowing the detection of biologically active compounds from complex mixtures (Visser 1979, Puri et al. 2006, Clark and Ray 2016, Morawo et al. 2016). In this current study, we screened Nasonov pheromone, its constituents, isoamyl acetate, and 7 HCAs in electroantennogram (EAG) bioassays. Here we report on the level of antennal response to the compounds in the honey bee.

\subsection{MATERIALS AND METHODS}

\subsubsection{Honey bees}

Bee colonies were maintained at the University of Nebraska-Lincoln Pollinator Garden Apiary using standard beekeeping practices. For the electroantennogram (EAG) recordings, worker bees were sampled on the same day from the uppermost super of the colony to avoid collecting newly emerged and nurse bees. Bees were kept in a well- 
ventilated box in the laboratory and, then anesthetized on ice to prepare the antennae for the EAG recordings.

\subsubsection{Chemicals}

All chemicals were purchased from Sigma-Aldrich Chemical Co. (St. Louis, MO). Synthetic Nasonov pheromone was purchased from Dadant \& Sons Beekeeping Equipment (Chatham, VA). Each chemical was diluted into either a hexane or ethanol carrier solvent at $10 \mu \mathrm{g} / \mu \mathrm{l}(1 \% \mathrm{w} / \mathrm{v})$ and stored at $4{ }^{\circ} \mathrm{C}$ prior to the EAG recordings. For the concentration response bioassays, a serial dilution of each chemical was prepared using either a hexane or ethanol carrier solvent. A $10 \mu \mathrm{l}$ aliquot of each chemical was applied to a strip of Whatman No. 1 filter paper (Sigma-Aldrich Chemical Co.) and, then, allowed to dry before inserting the chemical-treated paper into a Pasteur pipette $(15 \mathrm{~cm}$ long $)$ for the EAG recordings.

\subsubsection{Electroantennogram recordings}

Electroantennogram (EAG) recordings were conducted using Ag-AgCl electrodes inserted into glass capillary tubes filled with electrogel (Spectro 360, Parker Laboratory, NJ, USA). Thin walled glass capillary tubes (WPI, Sarasota Fl, USA) were pulled to a fine tip with a P-97 Flaming/Brown Micropipette puller (Sutter Instrument, Novato, CA, USA) using the following settings: Heat $=500 ;$ Pull $=25 ;$ Velocity $=200 ;$ Time $=500 ;$ Pressure $=500$. Bee antennae were dissected below the scape with micro scissors under a dissection microscope. The glass capillary tube was cut at the tip with micro forceps to fit the base of a bee antenna, while the other end of the capillary tube was attached to a reference 
electrode. The reference electrode was then positioned so that the tip of the excised antenna was near the recording electrode. A second glass capillary tube was prepared as described above to fit the tip of the bee antenna, and the other end of the capillary tube was attached to a recording electrode. The recording electrode was connected to a high-impedance D.C. amplifier (Ockenfels Syntech Gmbh, Kirchzarten, GER) and change in baseline voltage $(\mathrm{mV})$ was analyzed using EAG Pro 1.1 software (Syntech).

The change in baseline voltages of a bee antenna exposed to a compound was generated by inserting a Pasteur pipette with the chemical-treated filter paper into the opening of a glass mixing tube that was positioned perpendicular to the suspended bee antenna. This mixing tube provided a constant flow of humidified air over the prepared bee antenna. A 1-sec puff of air was passed through the Pasteur pipette and into the mixing tube to deliver the chemical to the bee antenna. An empty Pasteur pipette and hexane or ethanol treated filter paper served as a control and carrier solvent treatment, respectively. A total of 2-7 bee antennae were exposed to the compounds in a randomized order. The antennae were allowed to return to the baseline voltage before applying each compound. For the concentration response bioassays, a new antenna was used for each concentration of compound. The change in baseline voltage was recorded at 10 min intervals.

\subsubsection{Software and Data Analysis}

A baseline voltage of the bee antennae was generated prior to compound exposure using EAGPro software (Syntech). A lowest detection point was also generated that the program set indicating the voltage at the lowest point after a stimulus was applied. The absolute 
values of these two points for each stimulus were calculated and the baseline value was subtracted from the lowest detection point to give the change in baseline voltage $(\mathrm{mV})$. The change in baseline voltage was plotted into GraphPad Prism (La Jolla, CA) and statistically analyzed using an ANOVA with a Tukey's multiple comparison test.

\subsection{RESULTS}

Bee antennae were stimulated with pheromones and HCAs to examine their olfactory response to these compounds using EAG recordings. The change in baseline voltage for bee antennae exposed to the pheromones were observed as isoamyl acetate (low activity) $=$ nerol $=$ geraniol $<\operatorname{citral}=$ Nasonov $($ high activity $)($ Fig. 1A). A control (air only) and carrier solvent (hexane or ethanol only) treatment produced a $0-2 \mathrm{mV}$ change in baseline voltage of the bee antennae. The change in baseline voltages for bee antennae exposed Nasonov, citral, geraniol, nerol, and isoamyl acetate were 5.0 $\pm 0.4,4.4 \pm 0.4,3.6 \pm 0.4$, $2.5 \pm 0.4 \mathrm{mV}$, and $2.5 \pm 0.5 \mathrm{mV}$, respectively, significantly elevated compared to controls. There was not a significant difference in the responses of bee antennae exposed to Nasonov, citral, and geraniol; however, the change in baseline voltage of bee antennae exposed to Nasonov and citral was significantly higher than that of antennae exposed to nerol and isoamyl acetate. The change in baseline voltage of bee antennae exposed to nerol and isoamyl acetate was not significantly different than that provided by the control and solvent treatments. Furthermore, the change in baseline voltages for bee antennae exposed to citral, geraniol, and nerol was not significantly different from one another, which suggests similar olfactory response to the Nasonov pheromone constituents. 
The change in baseline voltages for bee antennae exposed to the HCAs at $1 \%$ were neither significantly different from one another nor were they significantly different from the control and solvent treatments (Fig. 1B). However, the HCAs at 100\% produced significantly higher changes in baseline voltage compared to the control and solvent treatments (Fig. 1C). Baseline voltage changes for bee antennae exposed to piperidine, pyrrolidine, and pyrrole at $100 \%$ were $22.4( \pm 5.0), 10.0( \pm 2.7), 1.0( \pm 0.5 \mathrm{SE}) \mathrm{mV}$, respectively. A significantly greater response was observed for piperidine compared to pyrrolidine and pyrrole; however, the changes in baseline voltage for pyrrolidine and pyrrole were not significantly difference from one another. The changes in baseline voltage for bee antennae exposed to DEET ranged from 1.0-1.3 mV, which were not significantly different from the control and solvent treatments (Fig. 1D).

EAG response amplitudes for bee antennae exposed to a concentration range of pyrrolidine and piperidine is shown in Fig. 2. The change in baseline voltages for bee antennae exposed to 1,5 , and $10 \%$, of both pyrrolidine and piperidine, were not significantly different from each other nor were they significantly different from control and solvent treatments. However, the change in baseline voltages for bee antennae exposed to 50 and $100 \%$ pyrrolidine were $11.2( \pm 2.3) \mathrm{mV}$ and $12.7( \pm 1.5) \mathrm{mV}$, respectively (Fig. 2A). This was significantly different from control and solvent treatments but not from each other. The change in baseline voltages for bee antennae exposed to 50 and 100\% piperidine were $12.7( \pm 2.2) \mathrm{mV}$ and $22( \pm 2.6) \mathrm{mV}$, respectively (Fig. $2 \mathrm{~B})$. This was significantly different from each other and from the control and solvent treatments. 


\subsection{DISCUSSION}

Pesticide use to ensure high crop yields in agriculture will continue to be needed and, thus, may continue to pose a threat to managed pollinators, such as honey bees (vanEngelsdorp and Meixner 2010). One way to mitigate this threat is by using repellents in combination with pesticide formulations to deter bees away from the treated crops (Atkins et al. 1975, Free et al. 1985). Previous studies have shown that bee pheromones and heterocyclic amines (HCAs) act as chemical deterrents to individual bees (Chapters 4 and 5). A better understanding of how these pheromones and HCAs are detected by the bees is an important step in the identification of repellents that could be used to protect bees from pesticide exposures. This study provides evidence for bee olfactory responses to pheromones and HCAs using electroantennogram (EAG) recordings. Bees antennae are responsive to the tested pheromones at $1 \%$ concentration, but do not respond to the HCAs below $10 \%$ concentration. A previous study reports these pheromones and HCAs to be comparable to DEET for deterring bees from a food source (Chapters 4 and 5). DEET is the gold standard for arthropod repellency testing (Lawrence et al. 2014) and was used as a positive control to validate the previously observed deterrent effects of the pheromones and HCAs (Chapters 4 and 5). However, the EAG responses of bee antennae to DEET up to $100 \%$ are not significantly different from the control and carrier solvent treatments. Bernier et al. (2005) report DEET to be a better contact repellent than a spatial repellent and, thus, the bee antennae might require direct contact with DEET rather than passing the compound across the antennae as described in this study. 
The pheromones Nasonov, geraniol, and citral appear to elicit an olfactory response by changing the baseline voltage of the bee antennae compared to that of the control and carrier solvent treatments. However, isoamyl acetate and nerol did not elicit an olfactory response at the concentrations tested in this study. It should be no surprise that these compounds are detected by bee antennae, since Nasonov, and its constituents geraniol and citral, is an aggregation pheromone with multiple purposes for a bee colony (Pickett et al. 1980). This pheromone is used to mark the hive entrance so that it can be found by other worker bees (Butler et al. 1970). It is also used in a bee swarm to keep the individuals together while they search for a new hive location (Morse and Boch 1971) or to locate a queen bee that has been lost from the colony (Boch and Morse 1974). These behaviors are presumed to be more difficult if the olfactory system was not the mode of detection for these pheromones. Williams et al. (1982) used EAG recordings to demonstrate that citral provided the strongest antennal response followed by geraniol and nerol, which is similar to the finding of this study. Masson and Arnold (1984) demonstrated that the strength of the EAG response is dependent on bee age, with younger bees having a stronger response to these pheromones compared to older bees exposed to the same concentrations. Furthermore, it was shown that isoamyl acetate elicits a stronger EAG response of bee antennae compared to Nasonov and its constituents (Masson and Arnold 1984). However, this observation is contrary to findings of this study where Nasonov and its constituents elicit a stronger EAG response of bee antennae compared to isoamyl acetate.

Isoamyl acetate is the primary component of alarm pheromone used by bees to mark threats to their hive (Boch et al. 1962). This activity results in the recruitment of additional bees 
to respond to the threat, which leads to an increase in isoamyl acetate released by the bees (Free and Simpson 1968). Roelofs (1984) demonstrated that an increase in stimulus concentration of isoamyl acetate correlates to increased amplitude of EAG responses. In this study, the bee antennae were exposed to pheromone concentrations at $1 \%$. The EAG responses reported by Masson and Arnold (1984) were elicited with pheromone concentrations at $100 \%$, which might explain the stronger response of the bee antennae to isoamyl acetate compared to the response to the other pheromones and those observed in this study. From a practical standpoint, a bee pheromone that excites a large portion of the colony might require a concentration threshold to be triggered before the occurrence of a biological response. While isoamyl acetate did not have a significant effect on the EAG responses of bee antennae in this study, it might be related to the low number of antennae used for the EAG recordings. Therefore, an increase in the number of antennae exposed to isoamyl acetate and nerol might decrease the variable responses detected with the EAG recordings.

The HCAs pyrrole, pyrrolidine, and piperidine at $1 \%$ concentration did not appear to elicit an olfactory response by changing the baseline voltage of the bee antennae compared to that of the control and carrier solvent treatments. Thus, these HCAs were tested at $100 \%$ concentration in an attempt to record an olfactory response of the bee antennae and, in fact, a change in the baseline voltage was observed with the bee antennae compared to that of the control and carrier solvent treatments. However, the use of physiologically irrelevant concentrations could force the odorant receptors to detect these HCAs at high concentrations. Piperidine elicited the strongest EAG response in the bee antennae. This 
observation correlates to the results of video-tracking experiments where bees exposed to a food source treated with piperidine were deterred away from that source (Chapter 5). Pyrrolidine elicited an EAG response less than that of piperidine and pyrrole. Thus, pyrrolidine and pyrrole were tested against bee antennae in a concentration-dependent manner, with EAG response detected at 10\%. These data suggest that the observed deterrence of bees from pyrrolidine- and pyrrole-treated food sources might not be an olfactory response. Tauxe et al. (2013) report that pyridine, a structure similar to piperidine, activates the $\mathrm{CO}_{2}$ receptor DmGr21a + DmGr63a and, in turn, the activation of this gustatory receptor causes aversion behavior in Drosophila melanogaster (Suh et al. 2004). While orthologs of these receptors have not been identified in the honey bee, it does suggest that gustatory reception might be the mode of detection for these compounds (Robertson and Wanner 2006a). Additionally, the video-tracking experiments with pyrrolidine and pyrrole demonstrate a deterrent effect on bee behavior whereas the EAG recordings are conducted with excised bee antennae. Therefore, EAG recordings of bees with intact antennae might confirm the mode of detection for bees exposed to these HCAs.

Overall, this study provides evidence that select pheromones elicit an olfactory response in bees that results in a deterrent effect from a treated food source. However, the deterrent effect observed with bees exposed to a food source treated with HCAs does not appear to be related to an olfactory response and, thus, requires further investigation into the sensory physiology of the bees to decipher the deterrent effects elicited by HCAs. The current study screened a select few of the HCAs that have been tested in previous deterrent assays (Chapter 5). It has been shown that altering the structure of the HCAs can alter the 
effectiveness of the compound. Screening additional HCA chemistries could lead to the discovery of a substituted version that is detected readily by the antennae. Additionally, discerning the mode of action of these compounds would allow for further investigation into receptor specificity. The bee genome encodes only 10 gustatory receptors genes, compared to 170 olfactory genes (Dunipace et al. 2001, Scott et al. 2001, Robertson and Wanner 2006b). Results potentially indicate that detection may be through contact which could allow for a quick screen of the relatively small number of genes that encode the receptors for gustatory reception. This could subsequently lead to the expression of the specific receptors for detection of HCAs into cell lines for an even higher throughput detection assay. 

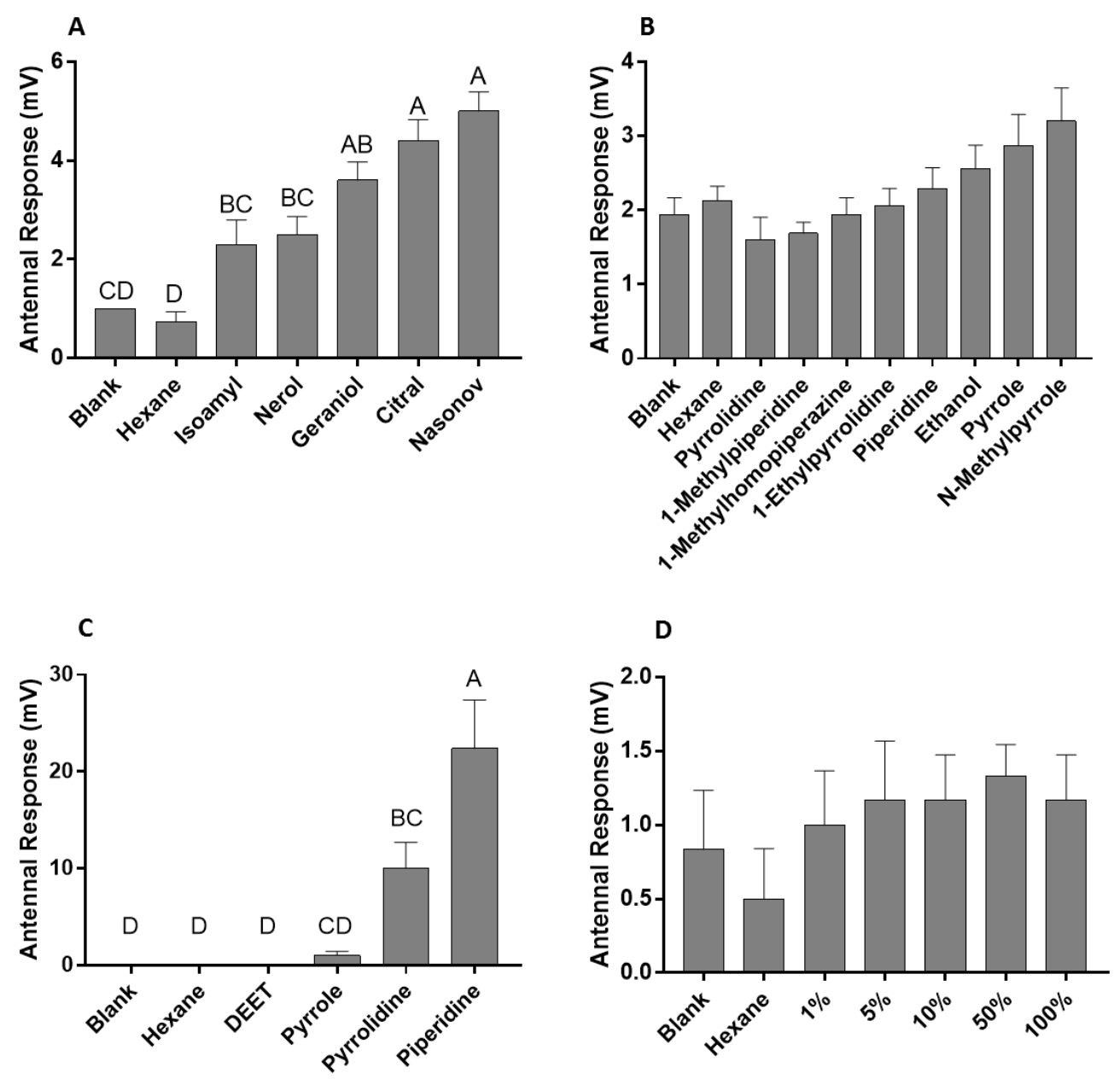

Figure 1. Electroantennogram (EAG) recordings of bee antennae exposed to pheromones, heterocyclic amines (HCAs), and DEET. The change in baseline voltage $(\mathrm{mV})$ was calculated by subtracting the absolute value of the baseline voltage from the absolute value of the response voltage. An empty Pasteur pipette and hexane or ethanol treated filter paper served as a control (blank) and carrier solvent treatment, respectively. (A) Bee antennae exposed to Nasonov, citral, geraniol, nerol, and isoamyl acetate pheromones at 1\% (w/v). (B) Bee antennae exposed to HCAs at $1 \%(w / v)$. (C) Bee antennae exposed to HCAs at 100\% (w/v). (D) Bee antennae exposed to DEET. Vertical bars represent the mean \pm standard error. Different letters above the bars indicate a significant difference between treatments using an ANOVA $(P<0.0001)$ with a Tukey's multiple comparison test 
P y rrolidine

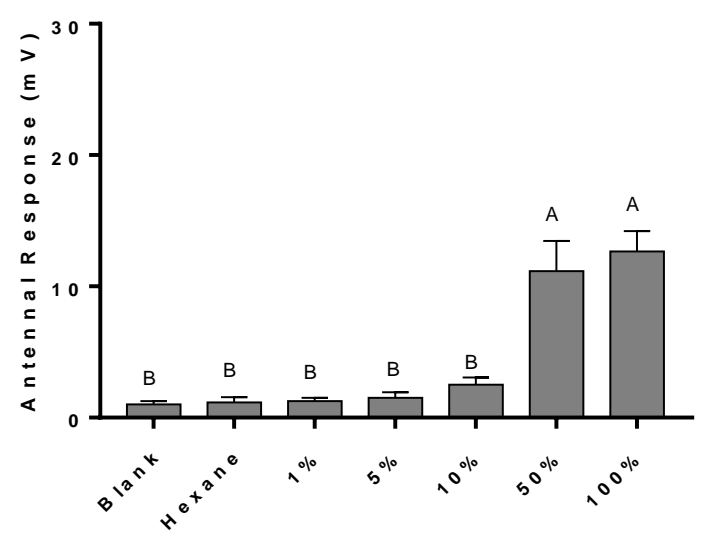

P ip e ridin e

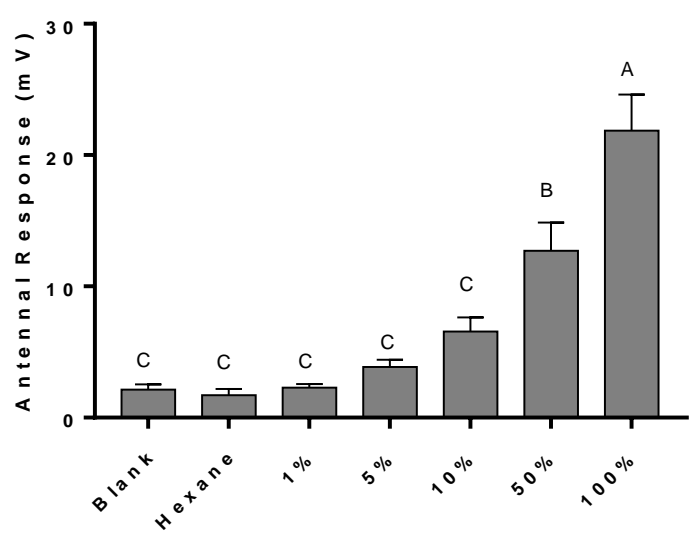

Figure 2. Electroantennogram (EAG) recordings of bee antennae exposed to the HCAs pyrrolidine and piperidine. The change in voltage was calculated by subtracting the absolute value of the baseline voltage from the absolute value of the response voltage. An empty Pasteur pipette and hexane treated filter paper served as a control (blank) and carrier solvent treatment, respectively. Vertical bars represent the mean \pm standard error. Different letters above the bars indicate a significant difference between treatments using an ANOVA $(P<0.0001)$ with a Tukey’s multiple comparison test. 


\subsection{REFERENCES}

(United States Environmental Protection Agency) . 2017. United States Environmental Protection Agency. (https://www.epa.gov/insect-repellents/skin-applied-repellentingredients).

Arn, H., E. Städler, and S. Rauscher. 1975. The electroantennographic detector - a selective and sensitive tool in the gas chromatographic analysis of insect pheromones. Zeitschrift fur Naturforsch. C. 30: 722-725.

Atkins, W., R. L. MacDonald, T. P. McGovern, M. Beroza, and E. A. Greywood-Hale. 1975. Repellent additives to reduce pesticide hazards to honeybees: Laboratory testing. J. Apic. Res. 14: 85-97.

Bernier, U. R., K. Posey, D. L. Kline, D. Barnard, and K. Chauhan; The United States of America as represented by the Secretary of Agriculture. 2012 Jun 26. Methods and compositions for repelling arthropods. U.S. patent 8,207,157B2.

Boch, R., and R. A. Morse. 1974. Discrimination of familiar and foreign queens by honey bee swarms. Ann. Entomol. Soc. Am. 67: 709-711.

Boch, R., D. A. Shearer, and B. C. Stone. 1962. Identification of iso-aamyl acetate as an active component in the sting pheromone of the honey bee. Nature. 195: 1018-1020.

Butler, C., D. Flethcher, and D. Watler. 1970. Hive entrance finding by honeybee (Apis mellifera) foragers. Anim. Behav. 18: 78-91.

Callbreath, J. 1900. The use of carbolic acid during spraying time. Glean. Bee Cult. 841.

Carroll, J. F., J. P. Benante, J. A. Klun, C. E. White, M. Debboun, J. M. Pound, and W. Dheranetra. 2008. Twelve-hour duration testing of cream formulations of three repellents against Amblyomma americanum. Med. Vet. Entomol. 22: 144-151. 
Clark, J. T., and A. Ray. 2016. Olfactory mechanisms for discovery of odorants to reduce insect-host contact. J. Chem. Ecol. 42: 919-930.

Collins, A. M., W. L. Rubink, J. I. Cuadriello Aguilar, and R. L. Hellmich II. 1996. Use of insect repellents for dispersing defending honey bees (Hymenoptera: Apidae). J. Econ. Entomol. 89: 608-613.

Dunipace, L., S. Meister, C. McNealy, and H. Amrein. 2001. Spatially restricted expression of candidate taste receptors in the Drosophila gustatory system. Curr. Biol. 11: $822-835$.

Free, J. B., J. A. Pickett, A. W. Ferguson, J. R. Simpkins, and M. C. Smith. 1985. Repelling foraging honeybees with alarm pheromones. J. Agric. Sci. 105: 255.

Free, J. B., and J. Simpson. 1968. The alerting pheromones of the honeybee. Z. vergl. Physiol. 61: 361-365.

Gallai, N., J. M. Salles, J. Settele, and B. E. Vaissière. 2009. Economic valuation of the vulnerability of world agriculture confronted with pollinator decline. Ecol. Econ. 68: $810-821$.

Hildebrand, J. G., and G. M. Shepherd. 1997. Mechanisms of olfactory discrimination: converging evidence for common principles across phyla. Annu Rev Neurosci. 20: 595-631.

Hoppe, H., and W. Ritter. 1988. The influence of the Nasonov pheromone on the recognition of house bees and foragers by Varroa jacobsoni. Apidologie. 19: 165172.

James, A. T., A. J. P. Martin, and G. H. Smith. 1952. Gas-liquid partition chromatography: the separation and micro-estimation of ammonia and the 
methylamines. Biochem. J. 52: 238-242.

Johansen, C. 1960. Bee repellent combined with dieldrin or sevin in bee poisoning tests in alfalfa. J. Econ. Entomol. 53: 1010-1012.

Katz, T. M., J. H. Miller, and A. A. Hebert. 2008. Insect repellents: historical perspectives and new developments. J. Am. Acad. Dermatol. 58: 865-871.

Klun, J. A., W. F. Schmidt, and M. Debboun. 2001. Stereochemical effects in an insect repellent. J. Med. Entomol. 38: 809-812.

Lawrence, K. L., N. L. Achee, U. R. Bernier, K. D. Mundal, and J. P. Benante. 2014. Field evaluations of topical arthropod repellents in North, Central, and South America. J. Med. Entomol. 51: 980-988.

Leal, W. S. 2013. Odorant reception in insects: roles of receptors, binding proteins, and degrading enzymes. Annu. Rev. Entomol. is. 58: 373-391.

Masson, C., and G. Arnold. 1984. Ontogeny, maturation and plasticity of the olfatory system in the workerbee. Insect. Physiol. 30(1): 7-14.

Moore, S. J., and M. Debboun. 2007. History of insect repellents, pp. 3-29. In Debboun, M., Frances, S.P., Strickman, D. (eds.), Insect Repellents Princ. Methods, Uses. CRC Press, Boca Raton.

Morawo, T., M. Burrows, and H. Fadamiro. 2016. Electroantennogram response of the parasitoid, Microplitis croceipes to host-related odors: the discrepancy between relative abundance and level of antennal responses to volatile compound. F1000Research. 5: 2725.

Morse, R. A., and R. Boch. 1971. Pheromone concert in swarming honey bees. Ann. Entomol. Soc. Amer. 64: 1414-1417. 
Mullin, C. A., M. Frazier, J. L. Frazier, S. Ashcraft, R. Simonds, D. VanEngelsdorp, and J. S. Pettis. 2010. High levels of miticides and agrochemicals in North American apiaries: Implications for honey bee health. PLoS One. 5: e9754.

Pickett, J. A., I. H. Williams, A. P. Martin, and M. C. Smith. 1980. Nasonov pheromone of the honey bee, Apis mellifera L. (Hymenoptera: Apidae) Part I. Chemical Characterization. J. Chem. Ecol. 6: 425-434.

Price, D. N., and M. S. Berry. 2008. Neurophysiological effects of naturally occurring defensive compounds on the freshwater snail Planorbis corneus: comparison with effects in insects. J. Chem. Ecol. 34: 994-1004.

Puri, S. N., M. J. Mendki, D. Sukumaran, K. Ganesan, and K. Sekhar. 2006. Electroantennogram and behavioral responses of Culex quinquefasciatus (Diptera: Culicidae) females to chemicals found in human skin emanations. J. Med. Entomol. 43: 207-213.

Riffel, J. A., and J. G. Hildebrand. 2016. Adaptive processing in the insect olfactory system, pp. 3-24. In von der Emde, G., Warrant, E. (eds.), Ecol. Anim. Senses. Springer International Publishing, Switzerland.

Robertson, H. M., and K. W. Wanner. 2006a. The chemoreceptor superfamily in the honey bee, Apis mellifera: Expansion of the odorant, but not gustatory, receptor family. Genome Res. 16: 1395-1403.

Robertson, H. M., and K. W. Wanner. 2006b. The chemoreceptor superfamily in the honey bee, Apis mellifera: Expansion of the odorant, but not gustatory, receptor family. Genome Res. 16: 1395-1403.

Roelofs, W. L., and A. Comeau. 1971. Sex pheromone perception: electroantennogram 
responses of the red-banded leaf roller moth. J. Insect Physiol. 17.

Schneider, D. 1957. Elektrophysiologische untersuchungen von chemo- und mechanorezeptoren der antenne des seidenspinners Bombyx mori L. Z. Vgl. Physiol. 40: 8-41.

Schneider, D. 1963. Electrophysiological investigation of insect olfaction, pp. 85-103. In Zotterman, Y. (ed.), Olfaction Tast. Elsevier, New York.

Scott, K., R. Brady, A. Cravchik, P. Morozov, A. Rzhetsky, C. Zuker, and R. Axel. 2001. A chemosensory gene family encoding candidate gustatory and olfactory receptors in Drosophila. Cell. 104: 661-673.

Solberg, V. B., T. A. Klein, K. R. McPherson, B. A. Bradford, J. R. Burge, and R. A. Wirtz. 1995. Field evaluation of deet and a piperidine repellent (A13- 37220) against Amblyomma americanum (Acari: Ixodidae). J. Med. Entomol. 32: 870-875.

Solomon, M. G., and K. J. M. Hooker. 1989. Chemical repellents for reducing pesticide hazard to honeybees in apple orchards. J. Apic. Res. 28: 223-227.

Suh, G. S. B., A. M. Wong, A. C. Hergarden, J. W. Wang, A. F. Simon, S. Benzer, R. Axel, and D. J. Anderson. 2004. A single population of olfactory sensory neurons mediates an innate avoidance behaviour in Drosophila. Nature. 431: 854-859.

Syntech. 2015. Electroantennography:a practical introduction. Ockenfels SYNTECH Gmbh, Kirchzarten, Ger.

vanEngelsdorp, D., and M. D. Meixner. 2010. A historical review of managed honey bee populations in Europe and the United States and the factors that may affect them. J. Invertebr. Pathol. 103: S80-S95.

Visser, J. H. 1979. Electroantennogram responses of the Colorado beetle, Leptinotarsa 
decemlineata, to plant volatiles. Entomol. Exp. Appl. 25: 86-97.

Warnhoff, E. W. 1998. When piperidine was a structural problem. Bull. Hist. Chem. 22: 29-34.

Woodrow, A., N. Green, H. Tucker, M. Schonhorst, and K. Hamilton. 1965. Evaluation of chemicals as honey bee attractants and repellents. J. Econ. Entomol. 58: $1094-1102$. 


\section{CHAPTER 8: CONCLUSIONS AND SUMMARY}

The crop pollination services and economic benefits that managed honey bee colonies provide to the apicultural and agricultural industry are immeasurable. However, the pest management practices of both industries can provide additional stress to bee colonies. There are several factors that can negatively affect bee colonies, including pathogens, parasites, weather, and pesticides. There are several studies that report pesticide exposures have detrimental effects on bees, including reduced immunocompetence and altered behaviors to individual paralysis and colony mortality. A number of pesticide residues, with multiple modes of action, are reported in the pollen, wax, and bees of hives, worldwide. There is need for additional protection practices to reduce the amount of pesticide exposures to bees rather than reliance on pesticide label recommendations. The primary goal of this study was to examine bee pheromonal compounds and heterocyclic amines (HCAs) for feeding deterrent and repellent activity against bees. This was achieved through the development of a laboratory video protocol for evaluating the biological activity of these compounds. A candidate compound (piperidine) was identified in the laboratory, and then tested using ta semi-field and field experiment. Finally, the olfactory response of bees exposed to these deterrent compounds was examined using electroantennogram recording experiments.

The development of the laboratory protocol proved difficult as many variations were attempted. The first attempt yielded information regarding the toxicity and behavioral effects elicited by the bee pheromonal compounds. This initial study found that a 
commonly used attractant (Nasonov) in beekeeping practices is toxic to bees within an enclosed arena. In addition to being toxic, these pheromonal compounds significantly inhibited the feeding activity of bees. While this initial experiment was unable to discern repellency, it did provide insight into additional effects that these compounds could potentially have on bees. This experiment also provided evidence that bee pheromonal compounds, at high concentrations, can deter bees from a food source.

Next, a video-tracking protocol was developed to record the movement of bees exposed to pheromonal compounds and HCAs. This protocol provided a quick, reliable evaluation of bees being deterred from a food source treated with the compounds. It was demonstrated that pheromonal compounds and HCAs have deterrent activities to bees comparable to that of DEET. This was an interesting finding as DEET is considered to be the gold standard in arthropod repellency screening and should be used for comparative analysis when screening novel arthropod repellents. While the development of a laboratory-based protocol for evaluating the repellent effects of select compounds was the goal, the protocol was unable to discern repellency based on the proper definition of directing movement away from a compound source. However, the protocol did provide evidence of bees are being deterred from the food source. This can be thought of as an expanded definition of repellency, as chemicals that mask an object or area prevent an insect from reaching the object or area of interest, or rather "repelling" the insect. The next step was to determine if these effects could be observed using semi-field experimentation. 
The high-tunnel and semi-field experiments were designed to determine if select compound would elicit the same deterrent effect on bees as observed in the laboratory. A single HCA was chosen to be used as a representative compound from previous laboratory experiments. The HCA piperidine deterred foraging bees away from a sugar feeder in the high-tunnel experiment. In addition, it was observed that piperidine deters foraging bees from melon flowers and knapweed in the semi-field experiment. These findings further validate the laboratory experiments for the identification of compounds with deterrent activities towards bees. The final experiment was conducted to examine the olfactory responses of bee antennae to the compounds as a prerequisite to identifying the mode of detection for the bees.

The use of electrophysiological methods has provided a great deal of insight into olfactory responses, and other physiological mechanisms, within insects. In this study, an electroantennogram recording method was used to determine whether bee antennae are stimulated by the laboratory- and field-tested deterrent compounds. The bee pheromonal compounds elicited responses in bee antennae; however, the HCAs did not elicit responses by the bee antennae at the same concentrations used for the laboratory and field experiments. In fact, the bee antennal responses did not occur until the concentration of the HCAs was increased by 10 -fold. This observation was interesting as the HCAs have been reported to elicit their repellent action via an olfactory response and a subsequent hyposmic or anosmic endpoint. It is possible that the HCAs are activating an olfactory receptor that is not located on antenna or through a contact mode of detection. This is 
potentially still acceptable for pollinator protection as it would still reduce the pesticide contact time with foraging bees.

The study has taken a discovery and development approach for screening chemical deterrents for the overall purpose of protecting bees from pesticide exposures. The compounds were screened using laboratory experimentation to observe behavior effects, and the most efficacious compound was evaluated in field experiments to verify observed laboratory effects were conserved in realistic field conditions. An electrophysiological technique was later used to examine the olfactory response of the bees to the compounds. The data collected can now be use for structure-activity relationship modeling to identify additional chemical deterrents for bees. Moreover, a primary goal of this study was to provide a much-needed laboratory protocol for evaluating the deterrent of effects of compounds to bees. Prior to the development of this protocol, much of what has been conducted in terms of bee repellency testing has been conducted using field experimentation. These field experiments can be expensive and time consuming for evaluating candidate compounds. A laboratory protocol to screen for deterrent effects allows for the quick elimination of non-deterrent compounds from a chemical library.

Ultimately, the pheromonal compounds and several HCAs were found to be effective deterrents for bees. However, there is still extensive research needed to develop longlasting and affordable compounds that can be added to a pesticide formulations that result in short-term deterrence of foraging bees from pesticide-treated crops. This study provides 
an important stepping point for future research to answer those questions. First, the efficacy of these compounds in combination with pesticides needs to be tested. This could be easily screened within the video-tracking protocol by adding pesticides concentrations to the repellent-sugar-agarose cubes. Additionally, if efficacy is conserved when in the presence of pesticides, the study could provide evidence that the bees are picking up less pesticide if mortality results are factored into the assay. The next important step for the development of a successful repellent additive would be to determine how long repellency would be desired. A compound that lasts too long could potentially harm bees by interfering with resource collection, while too short of a repellent effect would result in normal levels of pesticide exposure. This study did not specifically test for effective duration of these compounds; however, anecdotally, observations were made that suggest that the repellent effect of piperidine within the field experiments lasted less than an hour. This is most likely too short of an effective time to be useful in the field, however through use of structure-activity relationship models effective time could potentially be extended. Delivery is another variable that would need to be investigated. Ideally a successful candidate repellent would be able to be mixed in with a pesticide application so that it can be administered at the same time as the pesticide. This would both save time and money if this is achieved, however it may be that separate sprays would be needed. If the repellent cannot be mixed with the pesticide solution, then it may need to be administered after the pesticide application. Additionally, mixing the compounds could potentially result in loss of pesticide efficacy. Finally, the affordability of the repellent compound needs to be taken into consideration. The repellent would need to provide quantifiable benefits that outweigh the additional costs of utilization. 
As can be seen, there are several hurdles to overcome before a successful repellent can be developed for commercial use. The benefits of such a development could potentially be expanded beyond bees to protect all pollinators. The pheromonal and HCA compounds in this study have been found to be prospective candidates for further investigation into an effective pollinator repellent to provide protection against pesticide treated crops. 


\section{REFERENCES}

Andersson, M. N., C. Lofstedt, and R. D. Newcomb. 2015. Insect olfaction and the evolution of receptor tuning. Front. Ecol. Evol. 3: 1-14.

Barnard, D. R., U. R. Bernier, and K. H. Posey. 2002. Repellency of IR 3535, KBR3023, para-menthane-3, 8-diol, and deet to black salt marsh mosquitoes ( Diptera : Culicidae ) in the Everglades National Park. J. Med. Entomol. 39: 895-899.

Barton-Browne, L. 1977. Host-related responses and their suppression: some behavioral consideration, pp. 117-127. In Shorey, H., McKelvey, J. (eds.), Chem. Control Insect Behav. Theory Appl. John Wiley \& Sons, New York.

Benuszak, J., M. Laurent, and M.-P. Chauzat. 2017. The exposure of honey bees (Apis mellifera; Hymenoptera: Apidae) to pesticides: room for improvement in research. Sci. Total Environ. 587-588: 423-438.

Bernier, U. R., K. D. Furman, D. L. Kline, S. a Allan, and D. R. Barnard. 2005. Comparison of contact and spatial repellency of catnip oil and N,N-diethyl-3methylbenzamide (deet) against mosquitoes. J. Med. Entomol. 42: 306-311.

Biesmeijer, J. C., S. P. . Roberts, M. Reemer, R. Ohlemuller, M. Edward, T. Peeters, A. P. Schaffer, S. G. Potts, R. Kleukers, C. D. Thomas, J. Settele, and W. E. Kunin. 2006. Parallel declines in pollinators and insect-pollinated plants in Britain and the Netherlands. Science. 313: 351-354.

Bissinger, B. W., and R. M. Roe. 2010. Tick repellents: Past, present, and future. Pestic. Biochem. Physiol. 96: 63-79. 
Bissinger, B. W., and R. Mi. Roe. 2014. Tick repellent research, methods, and development, pp. 382-408. In Sonenshine, D.E., Roe, R.M. (eds.), Biol. Tick. Oxford University Press, New York.

Bogdanov, S., V. Kilchenmann, and A. Imdorf. 1997. Acaricide residues in beeswax and honey, pp. 239-246. In Mizrahi, A., Lensky, Y. (eds.), Bee Prod. Prop. Appl. Apitherapy. Springer Science, New York.

Bowden, R. M., S. Williamson, and M. D. Breed. 1998. Floral oils: their effect on nestmate recognition in the honey bee, Apis mellifera. Insectes Soc. 45: 209-214.

Briand, L., C. Nespoulous, V. Perez, J. J. Rémy, J. C. Huet, and J. C. Pernollet. 2000. Ligand-binding properties and structural characterization of a novel rat odorantbinding protein variant. Eur. J. Biochem. 267: 3079-3089.

Briand, L., N. Swasdipan, C. Nespoulous, V. Bézirard, F. Blon, J. C. Huet, P. Ebert, and J. C. Pernollet. 2002. Characterization of a chemosensory protein (ASP3c) from honeybee (Apis mellifera L.) as a brood pheromone carrier. Eur. J. Biochem. 269: $4586-4596$.

Calderone, N. W. 2012. Insect pollinated crops, insect pollinators and US agriculture: trend analysis of aggregate data for the period 1992-2009. PLoS One. 7: 24-28.

Carroll, S. P., and J. Loye. 2006. PMD, a registered botanical mosquito repellent with deet-like efficacy. J. Am. Mosq. Control Assoc. 22: 507-14.

Charlwood, D. 2003. Did Herodotus describe the first airborne use of mosquito repellent? Trends Parasitol. 19: 555. 
Cilek, J. E., J. L. Petersen, and C. E. Hallmon. 2004. Comparative efficacy of IR3535 and deet as repellents against adult Aedes aegypti and Culex quinquefasciatus. J. Am. Mosq. Control Assoc. 20: 299-304.

Dani, F. R., I. Iovinella, A. Felicioli, A. Niccolini, M. A. Calvello, M. G. Carucci, H. Qiao, G. Pieraccini, S. Turillazzi, G. Moneti, and P. Pelosi. 2010. Mapping the expression of soluble olfactory proteins in the honeybee. J. Proteome Res. 9: 18221833.

Debboun, M., and J. Wagman. 2004. In Vitro repellency of $\mathrm{N}, \mathrm{N}$-Diethyl-3methylbenzamide and N, N -Diethylphenylacetamide analogs against Aedes aegypti and Anopheles stephensi (Diptera: Culicidae). J. Med. Entomol. 41: 430-434.

Dethier, V. 1947. Chemical insect repellents and attractants. The Blakison Company, Philadelphia.

\section{Dulhunty, J. M., K. Yohannes, C. Kourleoutov, V. T. Manuopangai, M. K. Polyn, W.} J. Parks, and J. H. Bryan. 2000. Malaria control in central Malaita, Solomon Islands 2. Local perceptions of the disease and practices for its treatment and prevention. Acta Trop. 75: 185-196.

Eischen, F. a, R. H. Graham, and R. Cox. 2005. Regional distribution of Paenibacillus larvae subspecies larvae, the causative organism of American foulbrood, in honey bee colonies of the Western United States. J. Econ. Entomol. 98: 1087-1093.

Ellis, M. D., and F. P. Baxendale. 1997. Toxicity of seven monoterpenoids to tracheal mites (Acari: Tarsonemidae) and their honey bee (Hymenoptera: Apidae) hosts when applied as fumigants. J. Econ. Entomol. 90: 1087-1091. 
Elzen, P. J., F. a Eischen, J. R. Baxter, G. W. Elzen, and W. T. Wilson. 1999. Detection of resistance in US Varroa jacobsoni Oud. (Mesostigmata: Varroidae) to the acaricide fluvalinate. Apidologie. 30: 13-17.

Forêt, S., and R. Maleszka. 2006. Function and evolution of a gene family encoding odorant binding-like proteins in a social insect, the honey bee (Apis mellifera). Genome Res. 16: 1404-1413.

Fradin, M. S. 1998. Mosquitoes and mosquito repellents: a clinician's guide. Ann. Intern. Med. 128: 931-940.

Gouck, H., P. McGovern, and M. Beroza. 1967. Chemicals tested as space repellents against yellow-fever mosquitoes. I. Esters. J. Econ. Entomol. 60: 1587-90.

Goulson, D., E. Nicholls, C. Botías, and E. L. Rotheray. 2015. Bee declines driven by combined stress from parasites, pesticides, and lack of flowers. Science. 347: 1255957.

Hansson, B. S., M. C. Larsson, and W. S. Leal. 1999. Green leaf volatile-detecting olfactory receptor neurons display very high sensitivity and specificity in a scarab beetle. Physiol. Entomol. 24: 121-126.

Hill, C. A., A. N. Fox, R. J. Pitts, L. B. Kent, P. L. Tan, M. A. Chrystal, A. Cravchik, F. H. Collins, H. M. Robertson, and L. J. Zwiebel. 2002. G protein coupled receptors in Anopheles gambiae. Science. 298: 176-178.

Jacques, A., M. Laurent, M. Ribière-Chabert, M. Saussac, S. Bougeard, G. E. Budge, P. Hendrikx, and M.-P. Chauzat. 2017. A pan-European epidemiological study 
reveals honey bee colony survival depends on beekeeper education and disease control. PLoS One. 12: e0172591.

Johnson, R. M., H. S. Pollock, and M. R. Berenbaum. 2009. Synergistic interactions between in-hive miticides in Apis mellifera. J. Econ. Entomol. 102: 474-479.

Johnson, R. M., Z. Wen, M. A. Schuler, and M. R. Berenbaum. 2006. Mediation of pyrethroid insecticide toxicity to honey bees (Hymenoptera: Apidae) by cytochrome P450 monooxygenases. Berenbaum Source J. Econ. Entomol. J. Econ. Entomol. 99: $1046-1050$.

Kennedy, J. S. 1947. The excitant and repellent effects on mosquitos of sub-lethal contacts with DDT. Bull. Entomol. Res. 37: 593-607.

Khan, A. A., and H. I. Maibach. 1972. A study of insect repellents. 1. Effect on the flight and approach by Aedes aegypti. J. Econ. Entomol. 65: 1318-1321.

Klatt, B. K., A. Holzschuh, C. Westphal, Y. Clough, I. Smit, E. Pawelzik, and T. Tscharntke. 2014. Bee pollination improves crop quality, shelf life and commercial value. Proc. Biol. Sci. 281: 20132440.

Klein, A.-M., B. E. Vaissière, J. H. Cane, I. Steffan-Dewenter, S. A. Cunningham, C. Kremen, and T. Tscharntke. 2007. Importance of pollinators in changing landscapes for world crops. Proc. Biol. Sci. 274: 66, 95-96, 191.

Klier, M., and F. Kuhlow. 1976. Neue Insektenabwehrmittel-am Stickstoff disubstituierte beta-alaninderivate. J. Soc. Cosmet. Chem. 27: 141.

Larsson, M. C., and W. S. Leal. 1999. Olfactory receptor neurons specific to chiral sex 
pheromone components in male and female Anomala cuprea beetles ( Coleoptera: Scarabaeidae ). J. Comp. Physiol. A Neuroethol. Sens. Neural Behav. Physiol. 184: 353-359.

Laughlin, J. D., T. S. Ha, D. N. M. Jones, and D. P. Smith. 2008. Activation of pheromone-sensitive neurons is mediated by conformational activation of pheromone-binding protein. Cell. 133: 1255-1265.

Laurent, M., P. Hendrikx, M. Ribiere-Chabert, and M. Chauzat. 2016. A panEuropean epidemiological study on honeybee colony losses 2012-2014. Sophia Antipolis.

Leal, W. 2005. Pheromone reception. Top. Curr. Chem. 240: 1-36.

Lee, K. V., N. Steinhauer, K. Rennich, M. E. Wilson, D. R. Tarpy, D. M. Caron, R. Rose, K. S. Delaplane, K. Baylis, E. J. Lengerich, J. Pettis, J. A. Skinner, J. T. Wilkes, R. Sagili, and D. VanEngelsdorp. 2015. A national survey of managed honey bee 2013-2014 annual colony losses in the USA. Apidologie. 46: 292-305.

Lodesani, M., M. Colombo, and M. Spreafico. 1995. Ineffectiveness of Apistan ${ }^{\circledR}$ treatment against the mite Varroa jacobsoni Oud in several districts of Lombardy (Italy). Apidologie. 26: 67-72.

Madden, A. H., A. W. Lindquist, and E. F. Knipling. 1944. Tests of repellents against chiggers. J Econ Entomol. 37: 283-286.

Mayack, C., and D. Naug. 2009. Energetic stress in the honeybee Apis mellifera from Nosema ceranae infection. J. Invertebr. Pathol. 100: 185-188. 
McCabe, E., W. Barthel, S. Gertler, and S. Hall. 1954. Insect repellents: III. N,Ndiethlamides. J. Org. Chem. 19: 493-498.

Nikonov, A. A., G. Peng, G. Tsurupa, and W. S. Leal. 2002. Unisex pheromone detectors and pheromone-binding proteins in scarab beetles. Chem. Senses. 27: 495504.

Pelletier, J., A. Guidolin, Z. Syed, A. J. Cornel, and W. S. Leal. 2010. Knockdown of a mosquito odorant-binding protein involved in the sensitive detection of oviposition attractants. J. Chem. Ecol. 36: 245-248.

Reisenman, C. E., T. A. Christensen, W. Francke, and J. G. Hildebrand. 2004. Enantioselectivity of projection neurons innervating identified olfactory glomeruli. J. Neurosci. 24: 2602-2611.

Rettich, F. 1999. Laboratory and field evaluation of two new mosquito repellents. In Proc. 13th Eur. SOVE Meet. European Society of Vevtor Ecology, Ankara.

Robertson, H. M., C. G. Warr, and J. R. Carlson. 2003. Molecular evolution of the insect chemoreceptor gene superfamily in Drosophila melanogaster. Proc. Natl. Acad. Sci. U. S. A. 100 Suppl: 14537-14542.

Roeder, T. 1999. Octopamine in invertebrates. Prog. Neurobiol. 59: 533-561.

\section{Sakurai, T., H. Mitsuno, S. S. Haupt, K. Uchino, F. Yokohari, T. Nishioka, I.} Kobayashi, H. Sezutsu, T. Tamura, and R. Kanzaki. 2011. A single sex pheromone receptor determines chemical response specificity of sexual behavior in the silkmoth Bombyx mori. PLoS Genet. 7. 
Siede, R., M. König, R. Büchler, K. Failing, and H.-J. Thiel. 2008. A real-time PCR based survey on acute bee paralysis virus in German bee colonies. Apidologie. 39: $650-661$.

Silva, K. T. 1991. Ayurveda, malaria and the indigenous herbal tradition in Sri Lanka. Soc. Sci. Med. 33: 153-160.

Silvina, N., J. Florencia, P. Nicolás, P. Cecilia, P. Lucía, S. Abbate, C.-L. Leonidas, D. Sebastián, M. Yamandú, C. Verónica, and H. Horacio. 2017. Neonicotinoids transference from the field to the hive by honey bees: towards a pesticide residues biomonitor. Sci. Total Environ. 581: 25-31.

Smith, K. R., and S. Mehta. 2003. The burden of disease from indoor air pollution in developing countries: comparison of estimates. Int. J. Hyg. Environ. Health. 206: 279-289.

Steinhauer, N., K. Rennich, M. E. Wilson, D. M. Caron, E. J. Lengerich, J. Pettis, R. Rose, J. A. Skinner, D. R. Tarpy, J. T. Wilkes, and D. VanEngelsdorp. 2014. A national survey of managed honey bee 2012-2013 annual colony losses in the USA: results from the Bee Informed Partnership. J. Apic. Res. 53: 1-18.

Tawatsin, a, S. D. Wratten, R. R. Scott, U. Thavara, and Y. Techadamrongsin. 2001. Repellency of volatile oils from plants against three mosquito vectors. J. Vector Ecol. 26: $76-82$.

Taylor, K. S., G. D. Waller, and L. A. Crowder. 1987. Impairment of a classical conditioned response of the honey bee (Apis mellifera L.) by sublethal doses of synthetic pyrethroid insecticides. Apidologie. 18: 243-252. 
Yu, S. J. 2008. The Toxicology and Biochemistry of Insecticides. CRC Press, Boca Raton. 